\title{
Synthesis of the ABCDEF and FGHI ring system of yessotoxin and adriatoxin
}

\author{
Yuan Zhang and Jon D Rainier \\ Yessotoxin and adriatoxin are members of the polycyclic ether family of marine natural products. Outlined in this article is our \\ synthetic approach to two subunits of these targets. Central to our strategy is a coupling sequence that employs an olefinic-ester \\ cyclization reaction. As outlined, this sequence was used in two coupling sequences. First, it was used to merge the A, B- and \\ E, F-bicyclic precursors and in the process generate the C- and D-rings. Second, it was used to couple the F- and I-rings while \\ building the eight-membered G-ring and subsequently the $\mathrm{H}$-ring pyran.
}

The Journal of Antibiotics (2016) 69, 259-272; doi:10.1038/ja.2016.18; published online 9 March 2016

\section{INTRODUCTION}

Yessotoxin (1, YTX), a disulfated polycyclic ether natural product was first reported in 1987 by Murate, Yasumoto and co-workers from the digestive gland of the scallop Patinopecten yessoensis (Figure 1). ${ }^{1}$ It was subsequently discovered that YTX is produced by the dinoflagellates Protoceratium reticulatum, Lingulodinium polyedrum and Gonyaulax spinifera. ${ }^{2-4}$ YTX is acutely toxic to mice when administered intraperitoneally $\left(\mathrm{LD}_{50}=286 \mu \mathrm{g} \mathrm{kg}^{-1}\right.$ ) but not orally (up to $54 \mathrm{mg} \mathrm{kg}^{-1}$ ). ${ }^{5-9}$ The bioactivity of YTX ranges from modulating cytosolic calcium homeostasis, ${ }^{10}$ disrupting the degradation pathway of E-cadherin, ${ }^{11,12}$ inducing apoptotic cell death through the activation of caspases, ${ }^{13}$ to inducing lysosomal damage. ${ }^{14}$ Adriatoxin (2, ATX), a trisulfated analogue of YTX, was first isolated in 1998 from the digestive gland of the mussel Mytilus galloprovincialis collected in the Adriatic Sea. ${ }^{15}$ Structurally, YTX and ATX share the same A-J ring system, with YTX bearing an additional pyran (K-ring in YTX) and a hydrophobic side chain. Both compounds have attracted considerable interest from the organic synthesis community because of their challenging structures and intriguing properties. ${ }^{16-27}$ We have previously reported the syntheses of the $\mathrm{AB}, \mathrm{EF}$ and IJ ring systems of ATX. ${ }^{28}$ Our approach to the total synthesis of marine polycyclic ether natural products has largely focused on a convergent strategy that is centered around an olefinic-ester cyclization reaction that pairs cyclic ether subunits and leads to the generation of two additional rings. ${ }^{29-31}$ Herein, we describe our convergent synthesis of the ABCDEF and FGHI ring systems of YTX and ATX (3 and 4).

\section{MATERIALS AND METHODS}

NMR spectra were recorded on Varian Inova- $400 \mathrm{MHz}$, Varian Inova$500 \mathrm{MHz}$ or Varian VXR-500 MHz spectrometers. Chemical shifts were reported in $\delta$, p.p.m., relative to benzene (7.16), chloroform (7.27) or dichloromethane (5.32) as internal standards. Coupling constants, $J$, were reported in Hertz $(\mathrm{Hz})$ and refer to apparent peak multiplicities and not true coupling constants. Mass spectra were recorded at the Mass Spectrometry Facility at the Department of Chemistry of the University of Utah at Salt Lake City on a Finnigan MAT 95 mass spectrometer. IR spectra were recorded on a Bruker Tensor 27 FT-IR spectrometer. Optical rotations were obtained on a Perkin-Elmer Model 343 polarimerter (Na D line) using a microcell with $1 \mathrm{dm}$ path length. Solvents were purified according to the guidelines in Purification of Common Laboratory Chemicals (Perrin, Armarego and Perrin: Oxford, 1966). Dichloromethane, 2,6-lutidine, triethylamine, TMEDA, chlorobenzene and pyridine were distilled from $\mathrm{CaH}_{2}$. Tetrahydrofuran and diethyl ether were dried from the sodium ketyl of benzophenone and distilled before use. Zinc dust $(<10 \mu \mathrm{m}$, Aldrich) was activated by washing with $5 \%$ hydrochloric acid, $\mathrm{H}_{2} \mathrm{O}$, methanol, and ether and dried in vacuo overnight. All other reagents were used without further purification. Unless otherwise noted, all reactions were performed under a nitrogen atmosphere in flame-dried glassware using standard syringe, cannula and septa apparatus. Concentration refers to the removal of solvent under reduced pressure (house vacuum at ca. $20 \mathrm{~mm} \mathrm{Hg}$ ). Analytical thin-layer chromatography (TLC) was performed on silica gel plates $(0.25 \mathrm{~mm})$ precoated with a fluorescent indicator. Flash chromatography was performed using $40-63 \mu \mathrm{m}$ silica gel $(200 \times 400 \mathrm{mesh})$.

$(((2 R, 4 \mathrm{a} R, 5 \mathrm{a} S, 7 R, 8 S, 9 \mathrm{a} R, 10 \mathrm{a} S)-8$-allyl-4a-methyl-2-

phenyloctahydro-4H-pyrano $\left[2^{\prime}, 3^{\prime}: 5,6\right]$ pyrano[3,2-d] [1,3] dioxin-7-yl) oxy)(tert-butyl)dimethylsilane (6)

To a solution of 5 ( $25 \mathrm{mg}, 0.072 \mathrm{mmol})$ and 2,6-lutidine ( $25 \mu \mathrm{l}, 0.22 \mathrm{mmol})$ in $\mathrm{CH}_{2} \mathrm{Cl}_{2}(10 \mathrm{ml})$ was added TBSOTf $(25 \mu \mathrm{l}, 0.11 \mathrm{mmol})$. The reaction mixture was stirred for $2 \mathrm{~h}$ after which the reaction was quenched with sat. $\mathrm{NaHCO}_{3}$ $(5 \mathrm{ml})$. The aqueous phase was extracted with $\mathrm{CH}_{2} \mathrm{Cl}_{2}(3 \times 5 \mathrm{ml})$ and the extracts were combined, dried $\left(\mathrm{Na}_{2} \mathrm{SO}_{4}\right)$ and concentrated. Flash chromatography (8:1 hexanes: ethyl acetate) provided $30 \mathrm{mg}$ of $\mathbf{6}(90 \%)$ as a colorless oil. $R_{\mathrm{f}} 0.60$ (5:1 hexanes:ethyl acetate); $[\alpha]^{20} \mathrm{D}=-48.9^{\circ}\left(c=0.47\right.$, THF); ${ }^{1} \mathrm{H}$ NMR $\left(500 \mathrm{MHz}, \mathrm{C}_{6} \mathrm{D}_{6}\right) \delta 7.60(\mathrm{~d}, J=7.3 \mathrm{~Hz}, 2 \mathrm{H}), 7.19(\mathrm{t}, J=7.3 \mathrm{~Hz}, 2 \mathrm{H}), 7.13$ (t, $J=7.3 \mathrm{~Hz}, 1 \mathrm{H}), 6.14$ (dddd, $J=17.1,10.2,6.8,6.8 \mathrm{~Hz}, 1 \mathrm{H}), 5.40(\mathrm{~s}, 1 \mathrm{H})$, $5.22(\mathrm{bd}, J=17.1 \mathrm{~Hz}, 1 \mathrm{H}), 5.13(\mathrm{bd}, J=10.2 \mathrm{~Hz}, 1 \mathrm{H}), 3.92(\mathrm{~d}, J=10.0 \mathrm{~Hz}, 1 \mathrm{H})$, $3.53(\mathrm{~d}, J=10.0 \mathrm{~Hz}, 1 \mathrm{H}), 3.47$ (ddd, $J=10.7,7.3,4.9 \mathrm{~Hz}, 1 \mathrm{H}$ ), 3.38 (ddd, 

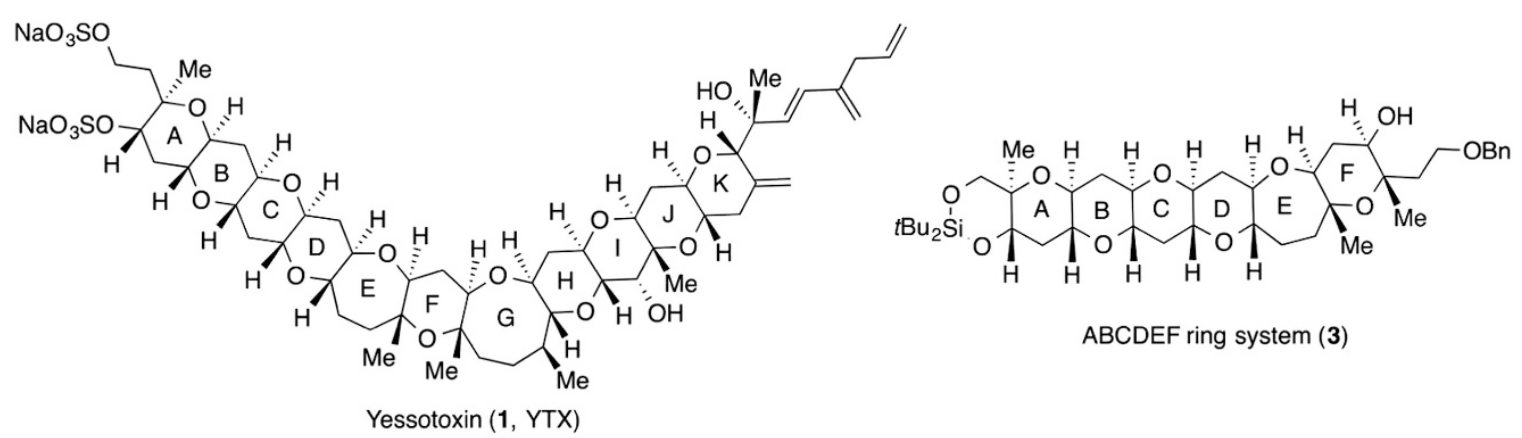

ABCDEF ring system (3)

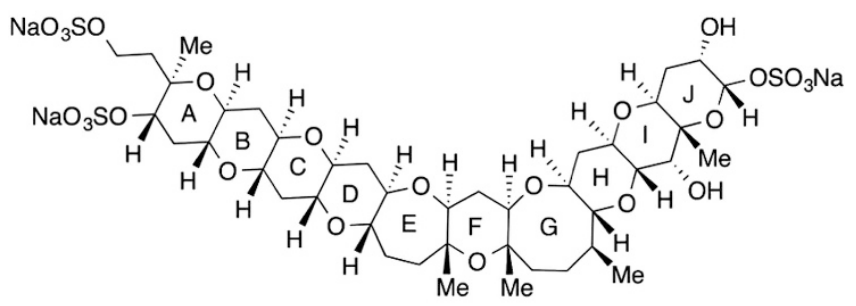

Adriatoxin (2, ATX)

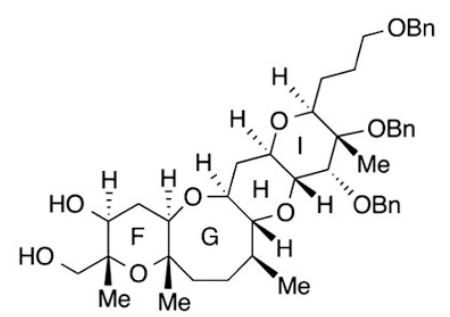

FGHI ring system (4)

Figure 1 Structures of yessotoxin, adriatoxin, the A-F and G-H ring systems.

$J=10.2,9.3,3.4 \mathrm{~Hz}, 1 \mathrm{H}), 3.35(\mathrm{dd}, J=12.5,3.5 \mathrm{~Hz}, 1 \mathrm{H}), 3.26(\mathrm{ddd}, J=8.8,8.8$, $2.9 \mathrm{~Hz}, 1 \mathrm{H}), 2.94(\mathrm{ddd}, J=10.2,9.8,4.9 \mathrm{~Hz}, 1 \mathrm{H}), 2.75-2.69(\mathrm{~m}, 1 \mathrm{H}), 2.35$ (ddd, $J=7.3,7.3,7.3 \mathrm{~Hz}, 1 \mathrm{H}), 2.31$ (ddd, $J=11.7,4.4,4.4 \mathrm{~Hz}, 1 \mathrm{H}), 2.19$ (ddd, $J=11.2,4.4,4.4 \mathrm{~Hz}, 1 \mathrm{H}), 1.88$ (ddd, $J=11.2,11.2,11.2 \mathrm{~Hz}, 1 \mathrm{H}), 1.43(\mathrm{~s}, 3 \mathrm{H})$, $0.93(\mathrm{~s}, 9 \mathrm{H}), 0.01(\mathrm{~s}, 3 \mathrm{H}),-0.01(\mathrm{~s}, 3 \mathrm{H}) ;{ }^{13} \mathrm{C}$ NMR $\left(125 \mathrm{MHz}, \mathrm{C}_{6} \mathrm{D}_{6}\right) \delta 138.5$, 135.3, 128.8, 128.2, 126.7, 116.8, 102.8, 82.6, 79.9, 78.2, 76.4, 70.3, 69.9, 69.6, 40.1, 36.4, 30.9, 25.8, 18.0, 15.2, -4.0, - 4.8; IR (neat) 2955, 2933, 2859, 1463, 1380, 1331, 1254, $1092 \mathrm{~cm}^{-1}$; ESI/MS $(\mathrm{m} / z)$ calcd for $\mathrm{C}_{26} \mathrm{H}_{41} \mathrm{O}_{5} \mathrm{Si} 461.3$ $\left(\mathrm{M}+\mathrm{H}^{+}\right)$, found 461.3.

2-((2R,4aR,5aS,7R,8S,9aR,10aS)-7-((tert-butyldimethylsilyl)oxy)-4a-methyl-2phenyloctahydro-4H-pyrano[2',3':5,6]pyrano[3,2-d][1,3]dioxin-8-yl)acetic acid (7). $\mathrm{O}_{3}$ was bubbled through a solution of $6(30 \mathrm{mg}, 0.065 \mathrm{mmol})$ in $\mathrm{CH}_{2} \mathrm{Cl}_{2}$ $(20 \mathrm{ml})$ at $-78^{\circ} \mathrm{C}$ until the reaction turned to a light blue color. Excess $\mathrm{O}_{3}$ was purged from the reaction mixture by bubbling $\mathrm{N}_{2}$ through it until the light blue color completely faded away. Triphenylphosphine $(51 \mathrm{mg}, 0.19 \mathrm{mmol})$ was then added and the reaction mixture was allowed to slowly warm to room temperature (RT). After $12 \mathrm{~h}$, the solution was concentrated under reduced pressure. Flash chromatography (50:1 to 5:1 hexanes:ethyl acetate) provided the corresponding aldehyde $(28 \mathrm{mg}, 92 \%)$ as a colorless oil.

To a solution of aldehyde obtained above $(28 \mathrm{mg}, 0.061 \mathrm{mmol})$ in THF $(3 \mathrm{ml})$ was successively added $t \mathrm{BuOH}(3 \mathrm{ml}), \mathrm{H}_{2} \mathrm{O}(3 \mathrm{ml}), 2$-Me-2-butene $(0.6 \mathrm{ml}), \mathrm{NaH}_{2} \mathrm{PO}_{4}(36 \mathrm{mg}, 0.30 \mathrm{mmol})$ and $\mathrm{NaClO}_{2}(27 \mathrm{mg}, 0.30 \mathrm{mmol})$. The resulting mixture was stirred at $\mathrm{RT}$ for $2 \mathrm{~h}$ after which the reaction was quenched with $\mathrm{H}_{2} \mathrm{O}(3 \mathrm{ml})$. The reaction mixture was extracted with ethyl acetate $(3 \times 5 \mathrm{ml})$ and the organic extracts were combined, dried $\left(\mathrm{Na}_{2} \mathrm{SO}_{4}\right)$ and concentrated. Flash chromatography (gradient of 1:1 hexanes:ethyl acetate to ethyl acetate) provided acid $7(19 \mathrm{mg}, 67 \%)$ as a colorless oil. $R_{\mathrm{f}} 0.30$ (2:1 hexanes:ethyl acetate); $[\alpha]^{20}{ }_{\mathrm{D}}=-52.2^{\circ} \quad(c=0.39$, THF $) ;{ }^{1} \mathrm{H} \quad \mathrm{NMR}$ $\left(500 \mathrm{MHz}, \mathrm{CD}_{2} \mathrm{Cl}_{2}\right) \delta$ 7.51-7.47 (m, 2H), 7.41-7.37 (m, 3H), $5.58(\mathrm{~s}, 1 \mathrm{H})$, $3.92(\mathrm{~d}, J=10.2 \mathrm{~Hz}, 1 \mathrm{H}), 3.69-3.60(\mathrm{~m}, 3 \mathrm{H}), 3.55$ (ddd, $J=10.7,9.3,4.9 \mathrm{~Hz}$, $1 \mathrm{H}), 3.48$ (ddd, $J=11.2,9.3,3.9 \mathrm{~Hz}, 1 \mathrm{H}), 3.22(\mathrm{ddd}, J=11.2,9.8,4.4 \mathrm{~Hz}, 1 \mathrm{H})$, 2.89 (dd, $J=15.6,2.9 \mathrm{~Hz}, 1 \mathrm{H}), 2.42$ (dd, $J=15.6,9.3 \mathrm{~Hz}, 1 \mathrm{H}$ ), 2.27 (ddd, $J=11.7,4.4,4.4 \mathrm{~Hz}, 1 \mathrm{H}), 2.20$ (ddd, $J=11.2,4.4,4.4 \mathrm{~Hz}, 1 \mathrm{H}$ ), 1.78 (ddd, $J=11.7,11.7,11.7 \mathrm{~Hz}, 1 \mathrm{H}), 1.53$ (ddd, $J=11.7,11.7,11.7 \mathrm{~Hz}, 1 \mathrm{H}), 1.52$ (s, 3H), $0.92(\mathrm{~s}, 9 \mathrm{H}), 0.14(\mathrm{~s}, 3 \mathrm{H}), 0.13(\mathrm{~s}, 3 \mathrm{H}) ;{ }^{13} \mathrm{C} \mathrm{NMR}\left(125 \mathrm{MHz}, \mathrm{CD}_{2} \mathrm{Cl}_{2}\right)$

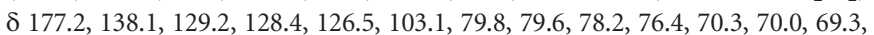
39.5, 37.7, 30.6, 25.7, 17.9, 15.1, - 4.1, - 4.9; IR (neat) 3259, 2954, 2861, 1714, $1463,1377,1256,1093 \mathrm{~cm}^{-1}$; ESI/MS $(\mathrm{m} / z)$ calcd for $\mathrm{C}_{25} \mathrm{H}_{37} \mathrm{O}_{7} \mathrm{Si} 477.3$ $\left(\mathrm{M}-\mathrm{H}^{+}\right)$, found 477.4.
2-((2R,4aR,5aS,7R,8S,9aR,11aS)-7-((4-methoxybenzyl)oxy)-8,9a-dimethyl-2phenyldecahydro-[1,3]dioxino[5,4-b]pyrano[2,3-f]oxepin-8-yl)ethan-1-ol (9). To a solution of $8(28 \mathrm{mg}, 0.075 \mathrm{mmol})$ in THF $(5 \mathrm{ml})$ at RT was added KH (30 mg 30\% dispersion in mineral oil, $0.23 \mathrm{mmol}$ ). After being allowed to stir for $10 \mathrm{~min}, \mathrm{DMPU}(4.5 \mu \mathrm{l}, 0.038 \mathrm{mmol}), \operatorname{PMBBr}(54 \mu \mathrm{l}, 0.38 \mathrm{mmol})$ and a catalytic amount of TBAI were added to the reaction mixture. After stirring overnight, the reaction mixture was quenched with sat. $\mathrm{NH}_{4} \mathrm{Cl}$ (aq., $5 \mathrm{ml}$ ). The aqueous phase was extracted with $\mathrm{CH}_{2} \mathrm{Cl}_{2}(3 \times 5 \mathrm{ml})$ and the combined organic extracts were dried $\left(\mathrm{Na}_{2} \mathrm{SO}_{4}\right)$ and concentrated. Flash chromatography (10:1 hexanes:ethyl acetate) gave a colorless oil, which was used in the next step without further purification.

$\mathrm{O}_{3}$ was bubbled through a solution of the PMB ether from the procedure outlined above in $\mathrm{CH}_{2} \mathrm{Cl}_{2}(20 \mathrm{ml})$ at $-78^{\circ} \mathrm{C}$ until the reaction mixture was a light blue color. Excess $\mathrm{O}_{3}$ was purged from the reaction mixture by bubbling $\mathrm{N}_{2}$ through it until the light blue color completely faded (ca. $10 \mathrm{~min}$ ). Triphenylphosphine $(59 \mathrm{mg}, 0.23 \mathrm{mmol}$ ) was then added and the reaction mixture was allowed to slowly warm to RT. After stirring for $12 \mathrm{~h}$, the resulting solution was concentrated under reduced pressure. Flash chromatography (50:1 to 5:1 hexanes:ethyl acetate) provided the corresponding aldehyde as a colorless oil. The aldehyde was used in the subsequent transformation without additional purification.

To a solution of the aldehyde obtained above in $\mathrm{MeOH}(5 \mathrm{ml})$ at $0{ }^{\circ} \mathrm{C}$ was added $\mathrm{NaBH}_{4}(8.5 \mathrm{mg}, 0.23 \mathrm{mmol})$. The reaction mixture was stirred for $2 \mathrm{~h}$, after which the reaction was quenched with acetone $(3 \mathrm{ml})$. The mixture was concentrated and the residue was purified using flash chromatography (4:1 hexanes:ethyl acetate) to give 9 as a colorless oil $(28 \mathrm{mg}, 74 \%$ over three steps). $R_{\mathrm{f}} 0.50$ (2:1 hexanes:ethyl acetate); $[\alpha]^{20}{ }_{\mathrm{D}}=-15.5^{\circ}(c=0.45$, THF); ${ }^{1} \mathrm{H}$ NMR $\left(500 \mathrm{MHz}, \mathrm{CD}_{2} \mathrm{Cl}_{2}\right) \delta$ 7.54-7.51 (m, 2H), 7.44-7.38 (m, 3H), 7.29 (d, $J=8.8 \mathrm{~Hz}, 2 \mathrm{H}), 6.94(\mathrm{~d}, J=8.3 \mathrm{~Hz}, 2 \mathrm{H}), 5.45(\mathrm{~s}, 1 \mathrm{H}), 4.64(\mathrm{~d}, J=11.2 \mathrm{~Hz}$, $1 \mathrm{H}), 4.41(\mathrm{~d}, J=11.2 \mathrm{~Hz}, 1 \mathrm{H}), 4.33(\mathrm{dd}, J=10.2,4.9 \mathrm{~Hz}, 1 \mathrm{H}), 3.83(\mathrm{~s}, 3 \mathrm{H})$, 3.84-3.78 (partially obscured m, $1 \mathrm{H}$ ), 3.61 (dd, $J=10.3,10.3 \mathrm{~Hz}, 1 \mathrm{H}$ ), 3.54 (ddd, $J=9.8,9.8,4.9 \mathrm{~Hz}, 1 \mathrm{H}), 3.50(\mathrm{dd}, J=11.7,4.4 \mathrm{~Hz}, 1 \mathrm{H}), 3.37(\mathrm{dd}$, $J=12.2,3.4 \mathrm{~Hz}, 1 \mathrm{H}), 3.15$ (br s, $1 \mathrm{H}), 2.30-2.17(\mathrm{~m}, 2 \mathrm{H}), 1.96-1.85(\mathrm{~m}, 4 \mathrm{H})$, 1.79 (ddd, $J=12.2,12.2,12.2 \mathrm{~Hz}, 1 \mathrm{H}), 1.74$ (ddd, $J=14.7,7.8,3.9 \mathrm{~Hz}, 1 \mathrm{H}$ ), $1.34(\mathrm{~s}, 3 \mathrm{H}), 1.33(\mathrm{~s}, 3 \mathrm{H}) ;{ }^{13} \mathrm{C} \mathrm{NMR}\left(125 \mathrm{MHz}, \mathrm{CD}_{2} \mathrm{Cl}_{2}\right) \delta$ 159.6, 138.3, 130.7, $129.5,129.1,128.4,126.4,113.9,100.9,83.2,80.4,79.2,78.9,78.1,74.8,70.8$, 69.7, 59.3, 55.5, 43.5, 39.5, 28.7, 28.5, 21.9, 21.0; IR (neat) 3448, 2946, 2870, $1613,1513,1458,1377,1248,1096 \mathrm{~cm}^{-1}$; ESI/MS $(\mathrm{m} / \mathrm{z})$ calcd for $\mathrm{C}_{29} \mathrm{H}_{38} \mathrm{O}_{7} \mathrm{Na}$ $521.2\left(\mathrm{M}+\mathrm{Na}^{+}\right)$, found 521.3. 
(2S,3R,4aS,6R,7S,9aR)-2-(2-(benzyloxy)ethyl)-6-(hydroxymethyl)-3-((4-methoxybenzyl)oxy)-2,9a-dimethyloctahydro-2H-pyrano[3,2-b]oxepin-7-ol (10). To a solution of $9(0.126 \mathrm{~g}, 0.253 \mathrm{mmol})$ in THF $(5 \mathrm{ml})$ at RT was added $t \mathrm{BuOK}$ $(0.50 \mathrm{ml}$ of $1.0 \mathrm{M}$ solution in THF, $0.50 \mathrm{mmol})$. The mixture was stirred for $30 \mathrm{~min}$ and then cooled to $0{ }^{\circ} \mathrm{C} . \mathrm{BnBr}(0.12 \mathrm{ml}, 1.01 \mathrm{mmol})$ was added slowly and the reaction mixture was stirred for $3 \mathrm{~h}$ before the reaction was quenched with sat. $\mathrm{NH}_{4} \mathrm{Cl}$ (aq., $5 \mathrm{ml}$ ). The aqueous phase was extracted with $\mathrm{CH}_{2} \mathrm{Cl}_{2}$ $(3 \times 5 \mathrm{ml})$ and the combined organic extracts were dried $\left(\mathrm{Na}_{2} \mathrm{SO}_{4}\right)$ and concentrated.

The residue from above was dissolved in $\mathrm{MeOH}(15 \mathrm{ml})$ and the mixture was cooled to $0{ }^{\circ} \mathrm{C}$. CSA ( $15 \mathrm{mg}, 0.065 \mathrm{mmol}$ ) was added and the reaction mixture was allowed to slowly warm to RT. The reaction was quenched with sat. $\mathrm{NaHCO}_{3}$ (aq., $10 \mathrm{ml}$ ) after $5 \mathrm{~h}$. The aqueous phase was extracted with ethyl acetate $(3 \times 10 \mathrm{ml})$ and the combined organic extracts were dried $\left(\mathrm{Na}_{2} \mathrm{SO}_{4}\right)$ and concentrated. Flash chromatography (1:1 hexanes:ethyl acetate) gave $\mathbf{1 0}$ as a colorless oil $\left(0.112 \mathrm{~g}, 89 \%\right.$ over two steps). $R_{\mathrm{f}} 0.60$ (ethyl acetate); $[\alpha]^{20}{ }_{\mathrm{D}}=-19.0^{\circ}(c=0.51, \mathrm{THF}) ;{ }^{1} \mathrm{H}$ NMR $\left(500 \mathrm{MHz}, \mathrm{CD}_{2} \mathrm{Cl}_{2}\right) \delta 7.40-7.30$ (m, $5 \mathrm{H}), 7.28(\mathrm{~d}, J=8.3 \mathrm{~Hz}, 2 \mathrm{H}), 6.90(\mathrm{~d}, J=8.8 \mathrm{~Hz}, 2 \mathrm{H}), 4.59(\mathrm{~d}, J=11.2 \mathrm{~Hz}$, $1 \mathrm{H}), 4.48(\mathrm{~s}, 2 \mathrm{H}), 4.38(\mathrm{~d}, J=10.7 \mathrm{~Hz}, 1 \mathrm{H}), 3.87(\mathrm{br} \mathrm{d}, J=5.5 \mathrm{~Hz}, 1 \mathrm{H}), 3.82$ $(\mathrm{s}, 3 \mathrm{H}), 3.71(\mathrm{br} \mathrm{s}, 1 \mathrm{H}), 3.64-3.60(\mathrm{~m}, 2 \mathrm{H}), 3.55-3.48(\mathrm{~m}, 3 \mathrm{H}), 3.44$ (dd, $J=11.2,4.2 \mathrm{~Hz}, 1 \mathrm{H}), 2.70$ (br s, $2 \mathrm{H}$ ), 2.17 (ddd, $J=11.7,3.9,3.9 \mathrm{~Hz}$, $1 \mathrm{H}), 1.95-1.76(\mathrm{~m}, 4 \mathrm{H}), 1.76-1.65(\mathrm{~m}, 2 \mathrm{H}), 1.54(\mathrm{ddd}, J=13.7,6.3,2.2 \mathrm{~Hz}$, $1 \mathrm{H}), 1.27(\mathrm{~s}, 6 \mathrm{H}) ;{ }^{13} \mathrm{C}$ NMR $\left(125 \mathrm{MHz}, \mathrm{CD}_{2} \mathrm{Cl}_{2}\right) \delta 159.4,139.2,131.1,129.4$, $128.5,127.9,127.6,113.9,85.7,79.6,79.3,76.5,75.5,73.0,71.7,70.8,66.7$, $64.5,55.4,41.7,36.2,28.8,27.9,22.3,20.2$; IR (neat) 3383, 2929, 2870, 1613, $1455,1375,1248,1090 \mathrm{~cm}^{-1}$; ESI/MS $(\mathrm{m} / \mathrm{z})$ calcd for $\mathrm{C}_{29} \mathrm{H}_{40} \mathrm{O}_{7} \mathrm{Na} 523.3$ $\left(\mathrm{M}+\mathrm{Na}^{+}\right)$, found 523.3 .

$(2 S, 3 R, 4 a S, 6 R, 7 S, 9 a R)-2-(2-($ benzyloxy $)$ ethyl)-3-((4-methoxybenzyl)oxy)-2,9adimethyl-6-(prop-2-yn-1-yl)octahydro-2H-pyrano[3,2-b]oxepin-7-ol (12). To a solution of $10(0.112 \mathrm{~g}, 0.224 \mathrm{mmol})$ and 2,6-lutidine $(0.104 \mathrm{ml}, 0.893 \mathrm{mmol})$ in $\mathrm{CH}_{2} \mathrm{Cl}_{2}(20 \mathrm{ml})$ at $-78^{\circ} \mathrm{C}$ was added trifluoromethanesulfonic anhydride $(40.0 \mu \mathrm{l}, 0.234 \mathrm{mmol})$. After $30 \mathrm{~min}$, TBSOTf $(77.0 \mu \mathrm{l}, 0.335 \mathrm{mmol})$ was added to the reaction mixture. The reaction mixture was allowed to warm slowly to RT before it was quenched with sat. $\mathrm{NaHCO}_{3}$ (aq., $10 \mathrm{ml}$ ). The aqueous phase was extracted with $\mathrm{CH}_{2} \mathrm{Cl}_{2}(3 \times 10 \mathrm{ml})$. The combined organic phase was dried $\left(\mathrm{Na}_{2} \mathrm{SO}_{4}\right)$ and concentrated to give a yellow oil. The oil was dissolved in a 10:1 mixture of hexanes:ethyl acetate, filtered through a plug of silica gel (10:1) and concentrated. The resulting colorless oil was used in the next transformation without additional purification.

To a solution of trimethylsilylacetylene $(0.168 \mathrm{ml}, 1.19 \mathrm{mmol})$ in THF $(10 \mathrm{ml})$ at $0{ }^{\circ} \mathrm{C}$ was added $n$ BuLi $(0.474 \mathrm{ml}$ of $2.50 \mathrm{M}$ solution in hexanes, $1.19 \mathrm{mmol}$ ). The reaction mixture was stirred at $0{ }^{\circ} \mathrm{C}$ for $30 \mathrm{~min}$ and then cooled to $-78^{\circ} \mathrm{C}$. A solution of 11 obtained from above and HMPA $(0.207 \mathrm{ml}$, $1.19 \mathrm{mmol})$ in THF $(5 \mathrm{ml})$ was transferred via cannula into the acetylide containing reaction mixture. The reaction mixture was stirred for $2 \mathrm{~h}$ after which the reaction was quenched with sat. $\mathrm{NH}_{4} \mathrm{Cl}$ (aq., $10 \mathrm{ml}$ ). The aqueous phase was extracted with $\mathrm{CH}_{2} \mathrm{Cl}_{2}(3 \times 10 \mathrm{ml})$. The combined organic phase was dried $\left(\mathrm{Na}_{2} \mathrm{SO}_{4}\right)$ and concentrated to give a dark brown oil, which was taken up in THF $(10 \mathrm{ml})$. To the resulting solution was added TBAF $(1.12 \mathrm{ml}$ of $1.0 \mathrm{M}$ solution in THF, $1.12 \mathrm{mmol}$ ) and the mixture was stirred at RT overnight before the reaction was quenched with sat. $\mathrm{NH}_{4} \mathrm{Cl}$ (aq., $10 \mathrm{ml}$ ). The aqueous phase was extracted with $\mathrm{CH}_{2} \mathrm{Cl}_{2}(3 \times 10 \mathrm{ml})$, the organic phases were combined, dried $\left(\mathrm{Na}_{2} \mathrm{SO}_{4}\right)$ and concentrated. Flash chromatography (4:1 hexanes:ethyl acetate) gave $\mathbf{1 2}$ as a colorless oil $(85.7 \mathrm{mg}, 75 \%$ three steps). $R_{\mathrm{f}} 0.50$ (2:1 hexanes:ethyl acetate); $[\alpha]^{20}{ }_{\mathrm{D}}=-21.6^{\circ}\left(c=0.42\right.$, THF); ${ }^{1} \mathrm{H}$ NMR $\left(500 \mathrm{MHz}, \mathrm{CD}_{2} \mathrm{Cl}_{2}\right) \delta 7.40-7.28(\mathrm{~m}, 5 \mathrm{H}), 7.27(\mathrm{~d}, J=8.8 \mathrm{~Hz}, 2 \mathrm{H}), 6.90$ $(\mathrm{d}, J=8.3 \mathrm{~Hz}, 2 \mathrm{H}), 4.58(\mathrm{~d}, J=10.8 \mathrm{~Hz}, 1 \mathrm{H}), 4.48(\mathrm{~s}, 2 \mathrm{H}), 4.38(\mathrm{~d}, J=11.2 \mathrm{~Hz}$, $1 \mathrm{H}), 4.08$ (br s, $1 \mathrm{H}), 3.81(\mathrm{~s}, 3 \mathrm{H}), 3.79$ (partially obscured ddd, $J=7.3,5.4$, $1.5 \mathrm{~Hz}, 1 \mathrm{H}), 3.63-3.59(\mathrm{~m}, 2 \mathrm{H}), 3.52(\mathrm{dd}, J=12.2,4.2 \mathrm{~Hz}, 1 \mathrm{H}), 3.42(\mathrm{dd}$, $J=11.7,3.9 \mathrm{~Hz}, 1 \mathrm{H}), 3.41$ (ddd, $J=16.6,5.4,2.4 \mathrm{~Hz}, 1 \mathrm{H}), 2.33$ (ddd, $J=17.1$, 7.8, $2.7 \mathrm{~Hz}, 1 \mathrm{H}$ ), 2.18-2.10 (partially obscured $\mathrm{m}, 1 \mathrm{H}$ ), 2.13 (ddd, $J=12.2,3.9$, $3.9 \mathrm{~Hz}, 1 \mathrm{H}), 2.10(\mathrm{t}, J=2.4 \mathrm{~Hz}, 1 \mathrm{H}), 1.94-1.75(\mathrm{~m}, 5 \mathrm{H}), 1.68$ (ddd, $J=12.2$, $12.2,12.2 \mathrm{~Hz}, 1 \mathrm{H}), 1.51(\mathrm{ddd}, J=13.2,5.9,2.9 \mathrm{~Hz}, 1 \mathrm{H}), 1.25(\mathrm{~s}, 6 \mathrm{H}) ;{ }^{13} \mathrm{C} \mathrm{NMR}$ $\left(125 \mathrm{MHz}, \mathrm{CD}_{2} \mathrm{Cl}_{2}\right) \delta 159.4,139.3,131.2,129.4,128.5,127.8,127.6,113.8$, 83.3, 81.2, 79.5, 78.5, 76.5, 75.4, 73.3, 73.0, 70.8, 70.1, 66.7, 55.4, 41.7, 35.9, 28.7, 26.7, 24.6, 22.2, 20.1; IR (neat) 3419, 2929, 2869, 2339, 1613, 1454, 1374,
1248, $1089 \mathrm{~cm}^{-1}$; ESI/MS $(\mathrm{m} / \mathrm{z})$ calcd for $\mathrm{C}_{31} \mathrm{H}_{40} \mathrm{O}_{6} \mathrm{Na} 531.3\left(\mathrm{M}+\mathrm{Na}^{+}\right)$, found 531.3.

(2S,3R,4aS, 6R,7S,9aR)-6-allyl-2-(2-(benzyloxy)ethyl)-3-((4-methoxybenzyl)oxy)2,9a-dimethyloctahydro-2H-pyrano[3,2-b]oxepin-7-ol (13). To a solution of 12 $(85.7 \mathrm{mg}, 0.169 \mathrm{mmol})$ in ethyl acetate $(10 \mathrm{ml})$ were added quinoline $(10 \mu \mathrm{l}$, $0.08 \mathrm{mmol}$ ) and Lindlar's Pd catalyst $(10 \mathrm{mg})$. The mixture was stirred under 1 $\mathrm{H}_{2}$ (1 atm) for $2 \mathrm{~h}$ before it was passed through a Celite plug with ethyl acetate. The filtrate was concentrated and flash chromatography (4:1 hexanes:ethyl acetate) gave 13 as a colorless oil $(78.8 \mathrm{mg}, 92 \%) . R_{\mathrm{f}} 0.55$ (2:1 hexanes:ethyl acetate); $[\alpha]^{20}{ }_{\mathrm{D}}=-14.9^{\circ}(c=0.43, \mathrm{THF}) ;{ }^{1} \mathrm{H}$ NMR $\left(500 \mathrm{MHz}, \mathrm{CD}_{2} \mathrm{Cl}_{2}\right)$ $\delta 7.38-7.28(\mathrm{~m}, 5 \mathrm{H}), 7.26(\mathrm{~d}, J=8.8 \mathrm{~Hz}, 2 \mathrm{H}), 6.90(\mathrm{~d}, J=8.3 \mathrm{~Hz}, 2 \mathrm{H}), 5.89$ (dddd, $J=17.1,10.3,6.8,6.8 \mathrm{~Hz}, 1 \mathrm{H}), 5.14-5.07(\mathrm{~m}, 2 \mathrm{H}), 4.58(\mathrm{~d}, J=11.2 \mathrm{~Hz}$, $1 \mathrm{H}), 4.47(\mathrm{~s}, 2 \mathrm{H}), 4.37(\mathrm{~d}, J=11.2 \mathrm{~Hz}, 1 \mathrm{H}), 3.89(\mathrm{br} \mathrm{d}, J=5.9 \mathrm{~Hz}, 1 \mathrm{H}), 3.80$ $(\mathrm{s}, 3 \mathrm{H}), 3.68$ (ddd, $J=6.4,6.4,1.4 \mathrm{~Hz}, 1 \mathrm{H}), 3.62-3.58(\mathrm{~m}, 2 \mathrm{H}), 3.47(\mathrm{dd}$, $J=12.2,4.2 \mathrm{~Hz}, 1 \mathrm{H}), 3.41(\mathrm{dd}, J=11.7,3.9 \mathrm{~Hz}, 1 \mathrm{H}), 2.27-2.15(\mathrm{~m}, 2 \mathrm{H}), 2.10$ (ddd, $J=12.2,4.4,3.9 \mathrm{~Hz}, 1 \mathrm{H}), 1.93-1.80(\mathrm{~m}, 4 \mathrm{H}), 1.78-1.69(\mathrm{~m}, 2 \mathrm{H}), 1.65$ (ddd, $J=12.2,12.2,12.2 \mathrm{~Hz}, 1 \mathrm{H}$ ), 1.49 (ddd, $J=13.2,5.3,2.7 \mathrm{~Hz}, 1 \mathrm{H}$ ), 1.24 $(\mathrm{s}, 6 \mathrm{H}) ;{ }^{13} \mathrm{C}$ NMR $\left(125 \mathrm{MHz}, \mathrm{CD}_{2} \mathrm{Cl}_{2}\right) \delta 159.4,139.3,135.4,131.2,129.4$, $128.5,127.8,127.5,116.9,113.8,84.7,79.6,78.5,76.5,75.4,73.7,72.9,70.7$, $66.7,55.4,41.7,39.4,35.9,28.8,26.7,22.3,20.1$; IR (neat) $3423,2929,1613$, $1513,1454,1374,1248,1087 \mathrm{~cm}^{-1}$; ESI/MS $(\mathrm{m} / \mathrm{z})$ calcd for $\mathrm{C}_{31} \mathrm{H}_{42} \mathrm{O}_{6} \mathrm{Na} 533.3$ $\left(\mathrm{M}+\mathrm{Na}^{+}\right)$, found 533.3.

(2S,3R,4aS,6R,7S,9aR)-6-allyl-2-(2-(benzyloxy)ethyl)-3-((4-methoxybenzyl)oxy)2,9a-dimethyloctahydro-2H-pyrano[3,2-b]oxepin-7-yl 2-((2R,4aR,5aS, 7R,8S,9aR, 10aS)-7-hydroxy-4a-methyl-2-phenyloctahydro-4H-pyrano [2,3':5,6]pyrano[3,2-d] [1,3]dioxin-8-yl)acetate (14). To a solution of acid 7 (36.0 mg, $0.0753 \mathrm{mmol})$ in THF $(8 \mathrm{ml})$ were added triethylamine $(45.9 \mu \mathrm{l}, 0.330 \mathrm{mmol})$ and $2,4,6-$ trichlorobenzoyl chloride $(35.3 \mu \mathrm{l}, 0.223 \mathrm{mmol})$. The reaction mixture was heated to $40^{\circ} \mathrm{C}$ and stirred for $2 \mathrm{~h}$ before being concentrated. A solution of alcohol $13(36.8 \mathrm{mg}, 0.0722 \mathrm{mmol})$ in toluene $(10 \mathrm{ml})$ was transferred via cannula to the resulting residue. DMAP $(42.3 \mathrm{mg}, 0.347 \mathrm{mmol})$ was then added and the reaction mixture was heated at $40{ }^{\circ} \mathrm{C}$ for $2 \mathrm{~h}$ after which the reaction was quenched with sat. $\mathrm{NaHCO}_{3}$ (aq., $10 \mathrm{ml}$ ). The aqueous phase was extracted with $\mathrm{CH}_{2} \mathrm{Cl}_{2}(3 \times 10 \mathrm{ml})$ and the organic extracts were combined, dried $\left(\mathrm{Na}_{2} \mathrm{SO}_{4}\right)$ and concentrated. Flash chromatography (10:1 hexanes:ethyl acetate) provided ester $14(67.8 \mathrm{mg}, 97 \%)$ as a colorless oil. $R_{\mathrm{f}} 0.25$ (5:1 hexanes:ethyl acetate); $[\alpha]^{20}{ }_{\mathrm{D}}=-14.8^{\circ}(c=0.30, \mathrm{THF}) ;{ }^{1} \mathrm{H}$ NMR $\left(500 \mathrm{MHz}, \mathrm{CD}_{2} \mathrm{Cl}_{2}\right)$ $\delta$ 7.46-7.43 $(\mathrm{m}, 2 \mathrm{H}), \quad 7.38-7.34(\mathrm{~m}, \quad 7 \mathrm{H}), \quad 7.32-7.27(\mathrm{~m}, \quad 1 \mathrm{H}), \quad 7.22$ $(\mathrm{d}, J=8.3 \mathrm{~Hz}, 2 \mathrm{H}), 6.86(\mathrm{~d}, J=8.8 \mathrm{~Hz}, 2 \mathrm{H}), 5.88$ (dddd, $J=17.1,10.3,6.8$, $6.8 \mathrm{~Hz}, 1 \mathrm{H}), 5.52(\mathrm{~s}, 1 \mathrm{H}), 5.13(\mathrm{dd}, J=18.1,1.0 \mathrm{~Hz}, 1 \mathrm{H}), 5.09(\mathrm{~d}, J=10.7 \mathrm{~Hz}$, $1 \mathrm{H}), 4.99(\mathrm{~d}, J=6.3 \mathrm{~Hz}, 1 \mathrm{H}), 4.57(\mathrm{~d}, J=10.7 \mathrm{~Hz}, 1 \mathrm{H}), 4.49(\mathrm{~d}, J=11.7 \mathrm{~Hz}$, $1 \mathrm{H}), 4.45(\mathrm{~d}, J=11.7 \mathrm{~Hz}, 1 \mathrm{H}), 4.36(\mathrm{~d}, J=10.7 \mathrm{~Hz}, 1 \mathrm{H}), 3.89(\mathrm{~d}, J=10.3 \mathrm{~Hz}$, $1 \mathrm{H}), 3.79(\mathrm{~s}, 3 \mathrm{H}), 3.75(\mathrm{dd}, J=6.2,6.2 \mathrm{~Hz}, 1 \mathrm{H}), 3.72-3.58(\mathrm{~m}, 5 \mathrm{H}), 3.55$ (ddd, $J=9.8,9.8,4.4 \mathrm{~Hz}, 1 \mathrm{H}$ ), 3.48 (partially obscured ddd, $J=10.8,9.3,3.9 \mathrm{~Hz}$, $1 \mathrm{H}), 3.44$ (partially obscured dd, $J=11.7,4.4 \mathrm{~Hz}, 1 \mathrm{H}), 3.36(\mathrm{dd}, J=12.2$, $3.9 \mathrm{~Hz}, 1 \mathrm{H}$ ), 3.19 (ddd, $J=10.7,9.3,4.4 \mathrm{~Hz}, 1 \mathrm{H}), 2.87$ (dd, $J=14.9,2.7 \mathrm{~Hz}$, $1 \mathrm{H}), 2.39(\mathrm{dd}, J=14.9,9.5 \mathrm{~Hz}, 1 \mathrm{H}), 2.31-2.23(\mathrm{~m}, 3 \mathrm{H}), 2.16(\mathrm{ddd}, J=11.2,3.9$, $3.9 \mathrm{~Hz}, 1 \mathrm{H}), 2.12(\mathrm{ddd}, J=12.2,3.9,3.9 \mathrm{~Hz}, 1 \mathrm{H}), 1.97-1.64(\mathrm{~m}, 8 \mathrm{H}), 1.59-1.48$ $(\mathrm{m}, 1 \mathrm{H}), 1.50(\mathrm{~s}, 3 \mathrm{H}), 1.25(\mathrm{~s}, 3 \mathrm{H}), 1.24(\mathrm{~s}, 3 \mathrm{H}), 0.92(\mathrm{~s}, 9 \mathrm{H}), 0.13(\mathrm{~s}, 3 \mathrm{H}), 0.11$ $(\mathrm{s}, 3 \mathrm{H}) ;{ }^{13} \mathrm{C}$ NMR $\left(125 \mathrm{MHz}, \mathrm{CD}_{2} \mathrm{Cl}_{2}\right) \delta 170.8,159.4,139.3,138.0,134.8$, $131.1,129.3,129.2,128.5,128.4,127.8,127.6,126.6,117.2,113.8,103.1,82.6$, $80.5,80.1,79.9,78.9,78.2,77.1,76.3,76.2,75.4,73.0,71.0,70.4,70.0,69.4$, $66.8,55.4,42.0,39.6,39.2,38.3,36.6,30.7,28.9,25.8,23.6,22.0,19.8,18.0$, $15.1,-4.1,-4.8$; IR (neat) 2932, 2859, 1735, 1613, 1514, 1456, 1378, $1250,1092 \mathrm{~cm}^{-1}$; ESI/MS $(\mathrm{m} / \mathrm{z})$ calcd for $\mathrm{C}_{56} \mathrm{H}_{78} \mathrm{O}_{12} \mathrm{SiNa} 993.5\left(\mathrm{M}+\mathrm{Na}^{+}\right)$, found 993.5 .

$(2 R, 4 a R, 5 a S, 7 R, 8 S, 9 a R, 10 a S)-8-(((4 a R, 5 a S, 7 R, 8 S, 9 a R, 11 a S)-8-(2-($ benzyloxy $)$ ethyl)-7-((4-methoxybenzyl)oxy)-8,9a-dimethyl-4,4a,5a,6,7,8,9a,10,11,11adecahydrodipyrano[3,2-b:2',3'-f]oxepin-2-yl)methyl)-4a-methyl-2-phenyloctahydro4 H-pyrano[2',3':5,6]pyrano[3,2-d][1,3]dioxin-7-ol (15). To a solution of $\mathrm{TiCl}_{4}$ $(0.245 \mathrm{ml}, 2.23 \mathrm{mmol})$ in $\mathrm{CH}_{2} \mathrm{Cl}_{2}(15 \mathrm{ml})$ at $0{ }^{\circ} \mathrm{C}$ was added THF $(1.18 \mathrm{ml}$, $13.4 \mathrm{mmol}$ ) dropwise. To the resulting yellow solution was added TMEDA $(2.03 \mathrm{ml}, 13.4 \mathrm{mmol})$ dropwise; the solution turned to a red-brown color. The ice bath was then removed and the mixture was allowed to warm for $15 \mathrm{~min}$. 
Activated $\mathrm{Zn}$ dust $(330 \mathrm{mg}, 5.08 \mathrm{mmol})$ and $\mathrm{PbCl}_{2}(74.0 \mathrm{mg}, 0.266 \mathrm{mmol})$ were then added. The resulting mixture went through a series of color changes from brown to green to purple and finally to blue-green over the course of 3-5 min. To the slurry was transferred a solution of ester $14(67.8 \mathrm{mg}, 0.0699 \mathrm{mmol})$ and $\mathrm{CH}_{3} \mathrm{CHBr}_{2}(0.200 \mathrm{ml}, 2.23 \mathrm{mmol})$ in $\mathrm{CH}_{2} \mathrm{Cl}_{2}(5 \mathrm{ml})$ via cannula. The reaction mixture was then heated to reflux for $30 \mathrm{~min}$ before it was cooled to $0{ }^{\circ} \mathrm{C}$. The reaction was quenched by adding sat. $\mathrm{K}_{2} \mathrm{CO}_{3}$ (aq., $1.0 \mathrm{ml}$ ) for $30 \mathrm{~min}$ and filtering the mixture. Concentration and flash chromatography (10:1 hexanes: ethyl acetate) gave $\mathbf{1 5}$ as a colorless oil $(32.9 \mathrm{mg}, 50 \%) . R_{\mathrm{f}} 0.20$ (5:1 hexanes: ethyl acetate); $[\alpha]^{20}{ }_{\mathrm{D}}=-40.3^{\circ}(c=0.29, \mathrm{THF}) ;{ }^{1} \mathrm{H} \mathrm{NMR}\left(500 \mathrm{MHz}, \mathrm{CD}_{2} \mathrm{Cl}_{2}\right) \delta$ 7.48-7.45 (m, 2H), 7.40-7.32 (m, 7H), 7.30-7.26 (m, 1H), $7.24(\mathrm{~d}, J=8.3 \mathrm{~Hz}$, $2 \mathrm{H}), 6.86(\mathrm{~d}, J=8.8 \mathrm{~Hz}, 2 \mathrm{H}), 5.56(\mathrm{~s}, 1 \mathrm{H}), 4.56(\mathrm{~d}, J=11.2 \mathrm{~Hz}, 1 \mathrm{H}), 4.52(\mathrm{br} \mathrm{d}$, $J=3.4 \mathrm{~Hz}, 1 \mathrm{H}), 4.47(\mathrm{~d}, J=11.7 \mathrm{~Hz}, 1 \mathrm{H}), 4.42(\mathrm{~d}, J=11.7 \mathrm{~Hz}, 1 \mathrm{H}), 4.37(\mathrm{~d}$, $J=11.2 \mathrm{~Hz}, 1 \mathrm{H}), 3.90(\mathrm{~d}, J=10.2 \mathrm{~Hz}, 1 \mathrm{H}), 3.78(\mathrm{~s}, 3 \mathrm{H}), 3.67-3.60(\mathrm{~m}, 2 \mathrm{H})$, 3.60-3.52 (m, 2H), 3.50-3.40 (m, 5H), $3.37(\mathrm{dd}, J=12.2,3.9 \mathrm{~Hz}, 1 \mathrm{H}), 3.31$ (ddd, $J=9.3,9.3,2.0 \mathrm{~Hz}, 1 \mathrm{H}), 3.12(\mathrm{ddd}, J=11.2,9.3,4.4 \mathrm{~Hz}, 1 \mathrm{H}), 2.54(\mathrm{~d}$, $J=15.1 \mathrm{~Hz}, 1 \mathrm{H}), 2.28($ br d, $J=16.6 \mathrm{~Hz}, 1 \mathrm{H}), 2.21(\mathrm{ddd}, J=11.2,4.4,4.4 \mathrm{~Hz}$, $1 \mathrm{H}), 2.16(\mathrm{ddd}, J=11.7,3.9,3.9 \mathrm{~Hz}, 1 \mathrm{H}), 2.13(\mathrm{ddd}, J=12.2,3.9,3.9 \mathrm{~Hz}, 1 \mathrm{H})$, 2.05-1.66 (m, 10H), $1.50(\mathrm{~s}, 3 \mathrm{H}), 1.47$ (ddd, $J=10.7,10.7,10.7 \mathrm{~Hz}, 1 \mathrm{H}), 1.24$ (s, 3H), 1.19 (s, 3H), $0.90(\mathrm{~s}, 9 \mathrm{H}), 0.10(\mathrm{~s}, 3 \mathrm{H}), 0.09$ (s, 3H); ${ }^{13} \mathrm{C}$ NMR $\left(125 \mathrm{MHz}, \mathrm{CD}_{2} \mathrm{Cl}_{2}\right) \delta 159.4,151.6,139.3,138.1,131.1,129.4,129.2,128.5$, $128.4,1279,127.6,126.5,113.8,103.0,94.8,80.6,80.0,79.9,79.7,79.1,78.8$, $78.0,77.0,76.4,75.6,73.0,70.8,70.7,69.9,69.5,66.5,55.4,41.9,40.0,39.9$, $35.9,30.7,29.7,29.4,28.8,25.8,23.5,22.5,18.0,15.1,-4.1,-4.8$; IR (neat) $2933,2859,1651,1513,1458,1377,1250,1093 \mathrm{~cm}^{-1}$; ESI/MS $(\mathrm{m} / \mathrm{z})$ calcd for $\mathrm{C}_{55} \mathrm{H}_{76} \mathrm{O}_{11} \mathrm{SiNa} 963.5\left(\mathrm{M}+\mathrm{Na}^{+}\right)$, found 963.6.

$(2 R, 4 a R, 5 a S, 7 R, 8 S, 9 a R, 11 a S)-8-(2-($ benzyloxy $)$ ethyl)-2-(((2R,4aR,5aS, 7R,8S,9aR, 10aS)-7-hydroxy-4a-methyl-2-phenyloctahydro-4H-pyrano [2',3':5,6] pyrano [3,2-d] [1,3]dioxin-8-yl)methyl)-7-((4-methoxybenzyl)oxy)-8,9a-dimethyldecahydrodipyrano[3,2-b:2',3'-f]oxepin-3(2H)-one (18). To a solution of $15(28.6 \mathrm{mg}$, $0.0304 \mathrm{mmol})$ in $\mathrm{CH}_{2} \mathrm{Cl}_{2}(5 \mathrm{ml})$ at $-78^{\circ} \mathrm{C}$ was added a solution of dimethyl dioxirane $\left(0.61 \mathrm{ml}\right.$ of $0.10 \mathrm{M}$ solution in $\left.\mathrm{CH}_{2} \mathrm{Cl}_{2}, 0.061 \mathrm{mmol}\right)$ dropwise. The reaction mixture was warmed to $0{ }^{\circ} \mathrm{C}$ and then concentrated. The resulting residue was dissolved in $\mathrm{CH}_{2} \mathrm{Cl}_{2}(5 \mathrm{ml})$ and cooled to $-78^{\circ} \mathrm{C}$. To this was added a solution of DIBALH $(0.304 \mathrm{ml}$ of $1.0 \mathrm{M}$ solution in THF, $0.30 \mathrm{mmol})$. After stirring for $2 \mathrm{~h}$, the reaction was quenched with sat. $\mathrm{NH}_{4} \mathrm{Cl}$ (aq., $3 \mathrm{ml}$ ) and allowed to warm to RT. A solution of sat. potassium sodium tartrate solution (aq., $10 \mathrm{ml}$ ) was added and the resulting mixture was stirred for $30 \mathrm{~min}$. The aqueous phase was extracted with $\mathrm{CH}_{2} \mathrm{Cl}_{2}(3 \times 10 \mathrm{ml})$ and the combined organic phase was dried $\left(\mathrm{Na}_{2} \mathrm{SO}_{4}\right)$ and concentrated. Flash chromatography (5:1 hexanes:ethyl acetate) provided major alcohol 16 (17.5 mg, 60\%) and minor alochol $(6.1 \mathrm{mg}, 21 \%)$ both as colorless oil.

To a solution of $16(17.5 \mathrm{mg}, 0.0183 \mathrm{mmol})$ in $\mathrm{CH}_{2} \mathrm{Cl}_{2}(5 \mathrm{ml})$ was added activated $4 \AA \mathrm{MS}(20 \mathrm{mg}), \mathrm{NMO}(21.4 \mathrm{mg}, 0.183 \mathrm{mmol})$ and TPAP (1 mg, $0.003 \mathrm{mmol}$ ). The reaction mixture was stirred at RT for $2 \mathrm{~h}$ before being concentrated. Flash chromatography (5:1 hexanes:ethyl acetate) gave ketone 18 as a colorless oil (17.4 mg, 100\%). $R_{\mathrm{f}} 0.55$ (3:1 hexanes:ethyl acetate); $[\alpha]^{20}{ }_{\mathrm{D}}=-42.0^{\circ} \quad(c=0.10, \quad \mathrm{THF}) ; \quad{ }^{1} \mathrm{H} \quad \mathrm{NMR} \quad\left(500 \mathrm{MHz}, \mathrm{C}_{6} \mathrm{D}_{6}\right) \quad \delta \quad 7.60$ $(\mathrm{d}, J=7.8 \mathrm{~Hz}, 2 \mathrm{H}), 7.33(\mathrm{~d}, J=7.8 \mathrm{~Hz}, 2 \mathrm{H}), 7.26-7.06(\mathrm{~m}, 9 \mathrm{H}), 6.79(\mathrm{~d}$, $J=7.8 \mathrm{~Hz}, 2 \mathrm{H}), 5.39(\mathrm{~s}, 1 \mathrm{H}), 4.44(\mathrm{~d}, J=11.2 \mathrm{~Hz}, 1 \mathrm{H}), 4.41(\mathrm{~d}, J=11.2 \mathrm{~Hz}$, $1 \mathrm{H}), 4.36(\mathrm{~d}, J=11.7 \mathrm{~Hz}, 1 \mathrm{H}), 4.25(\mathrm{~d}, J=11.2 \mathrm{~Hz}, 1 \mathrm{H}), 4.08(\mathrm{dd}, J=7.3$, $3.4 \mathrm{~Hz}, 1 \mathrm{H}), 3.92(\mathrm{~d}, J=10.2 \mathrm{~Hz}, 1 \mathrm{H}), 3.78-3.70(\mathrm{~m}, 2 \mathrm{H}), 3.66-3.60(\mathrm{~m}, 1 \mathrm{H})$, 3.56-3.50 (partially obscured $\mathrm{m}, 1 \mathrm{H}$ ), 3.53 (partially obscured d, $J=10.2 \mathrm{~Hz}$, $1 \mathrm{H}), 3.45-3.25(\mathrm{~m}, 4 \mathrm{H}), 3.28(\mathrm{~s}, 3 \mathrm{H}), 3.16(\mathrm{ddd}, J=8.3,8.3,4.4 \mathrm{~Hz}, 1 \mathrm{H})$, $3.08-3.00(\mathrm{~m}, 2 \mathrm{H}), 2.97(\mathrm{dd}, J=15.6,6.4 \mathrm{~Hz}, 1 \mathrm{H}), 2.76(\mathrm{ddd}, J=13.2,7.8$, $2.4 \mathrm{~Hz}, 1 \mathrm{H}), 2.36-2.22(\mathrm{~m}, 3 \mathrm{H}), 2.16-2.00(\mathrm{~m}, 4 \mathrm{H}), 2.00-1.88(\mathrm{~m}, 2 \mathrm{H})$, 1.86-1.62 (m, 6H), $1.43(\mathrm{~s}, 3 \mathrm{H}), 1.33(\mathrm{~s}, 3 \mathrm{H}), 1.18(\mathrm{~s}, 3 \mathrm{H}), 0.98(\mathrm{~s}, 9 \mathrm{H}), 0.06$ $(\mathrm{s}, 3 \mathrm{H}), 0.02(\mathrm{~s}, 3 \mathrm{H}) ;{ }^{13} \mathrm{C}$ NMR $\left(125 \mathrm{MHz}, \mathrm{C}_{6} \mathrm{D}_{6}\right) \delta 205.1,159.6,139.4,138.4$, $131.0,129.2,128.9,128.4,128.2,127.4,126.7,114.0,102.8,81.0,80.2,80.1$, $79.8,79.5,79.4,78.9,78.3,76.9,76.4,75.7,73.0,71.7,70.6,69.9,69.5,66.5$, $54.6,46.1,42.2,40.2,39.6,36.7,33.3,31.0,30.1,29.2,28.8,25.8,24.8,22.8$, 22.6, 18.0, 15.1, -4.1, -4.8; IR (neat) 2931, 2858, 1727, 1513, 1460, 1377, 1250, $1092 \mathrm{~cm}^{-1}$; ESI/MS $(\mathrm{m} / \mathrm{z})$ calcd for $\mathrm{C}_{55} \mathrm{H}_{78} \mathrm{O}_{12} \mathrm{SiK} 995.5\left(\mathrm{M}+\mathrm{K}^{+}\right)$, found 995.5.
$(2 R, 3 S, 4 a R, 5 a S, 7 R, 8 S, 9 a R, 11 a S)-8-(2-($ benzyloxy $)$ ethyl)-2-(((2R,4aR,5aS,7R,8S,9aR, 10aS)-7-hydroxy-4a-methyl-2-phenyloctahydro-4H-pyrano [2',3':5,6]pyrano [3,2-d] [1,3]dioxin-8-yl)methyl)-7-((4-methoxybenzyl)oxy)-8,9a-dimethyldodecahydrodipyrano[3,2-b:2',3'-f]oxepin-3-yl acetate (17). To a solution of 16 (25.6 mg, $0.0267 \mathrm{mmol})$ in $\mathrm{CH}_{2} \mathrm{Cl}_{2}(8 \mathrm{ml})$ were added $\mathrm{Et}_{3} \mathrm{~N}(37.2 \mu \mathrm{l}, 0.267 \mathrm{mmol}), \mathrm{Ac}_{2} \mathrm{O}$ $(12.6 \mu \mathrm{l}, 0.133 \mathrm{mmol})$ and DMAP $(3.3 \mathrm{mg}, 0.027 \mathrm{mmol})$. The reaction mixture was stirred for $2 \mathrm{~h}$ before the reaction was quenched with sat. $\mathrm{NaHCO}_{3}$ (aq., $5 \mathrm{ml})$. After separation the aqueous phase was extracted with $\mathrm{CH}_{2} \mathrm{Cl}_{2}(3 \times 5 \mathrm{ml})$ and the combined organic phase was dried $\left(\mathrm{Na}_{2} \mathrm{SO}_{4}\right)$ and concentrated. Flash chromatography (5:1 hexanes:ethyl acetate) provided 17 as a colorless oil (21.7 mg, 81\%). $R_{\mathrm{f}} 0.60$ (3:1 hexanes:ethyl acetate); $[\alpha]^{20}{ }_{\mathrm{D}}=-24.9^{\circ}(c=0.24$, THF); ${ }^{1} \mathrm{H} \quad \mathrm{NMR}\left(500 \mathrm{MHz}, \mathrm{C}_{6} \mathrm{D}_{6}\right) \quad \delta \quad 7.60$ (d, $\left.J=7.3 \mathrm{~Hz}, \quad 2 \mathrm{H}\right), \quad 7.33$ (d, $J=7.3 \mathrm{~Hz}, 2 \mathrm{H}), 7.24-7.08(\mathrm{~m}, 8 \mathrm{H}), 6.79(\mathrm{~d}, J=7.8 \mathrm{~Hz}, 2 \mathrm{H}), 5.39(\mathrm{~s}, 1 \mathrm{H})$, 4.99 (ddd, $J=10.7,10.7,4.4 \mathrm{~Hz}, 1 \mathrm{H}), 4.52(\mathrm{~d}, J=11.2 \mathrm{~Hz}, 1 \mathrm{H}), 4.42$ (d, $J=11.2 \mathrm{~Hz}, 1 \mathrm{H}), 4.37$ (d, $J=13.2 \mathrm{~Hz}, 1 \mathrm{H}), 4.34(\mathrm{~d}, J=12.2 \mathrm{~Hz}, 1 \mathrm{H}), 3.92$ $(\mathrm{d}, J=9.8 \mathrm{~Hz}, 1 \mathrm{H}), 3.76(\mathrm{dd}, J=15.6,7.3 \mathrm{~Hz}, 1 \mathrm{H}), 3.68-3.50(\mathrm{~m}, 6 \mathrm{H})$, $3.50-3.37(\mathrm{~m}, 2 \mathrm{H}), 3.30(\mathrm{~s}, 3 \mathrm{H}), 3.20-3.01(\mathrm{~m}, 4 \mathrm{H}), 2.77$ (ddd, $J=11.7,4.4$, $4.4 \mathrm{~Hz}, 1 \mathrm{H}), 2.44(\mathrm{ddd}, J=12.2,3.9,3.9 \mathrm{~Hz}, 1 \mathrm{H}), 2.38-2.30(\mathrm{~m}, 2 \mathrm{H})$, 2.16-2.02 (m, 3H), 2.02-1.94 (m, 2H), $1.90(\mathrm{ddd}, J=11.7,11.7,11.7 \mathrm{~Hz}$, $1 \mathrm{H}), 1.85-1.76(\mathrm{~m}, 3 \mathrm{H}), 1.75(\mathrm{~s}, 3 \mathrm{H}), 1.72-1.53(\mathrm{~m}, 3 \mathrm{H}), 1.45(\mathrm{~s}, 3 \mathrm{H}), 1.32$ (s, 3H), $1.20(\mathrm{~s}, 3 \mathrm{H}), 0.98(\mathrm{~s}, 9 \mathrm{H}), 0.09(\mathrm{~s}, 3 \mathrm{H}), 0.03(\mathrm{~s}, 3 \mathrm{H}) ;{ }^{13} \mathrm{C}$ NMR (125 MHz, $\mathrm{C}_{6} \mathrm{D}_{6}$ ) $\delta 169.2,159.6,139.4,138.5,131.2,129.3,128.8,128.4,128.2$, $127.4,126.7,114.0,102.8,81.5,81.1,79.9,79.7,79.3,79.1,78.3,77.0,76.4$, 75.7, 73.0, 71.1, 70.6, 69.9, 69.6, 66.6, 54.7, 42.2, 40.4, 39.8, 37.7, 34.5, 30.9, $30.1,29.5,29.0,25.9,22.7,22.6,20.7,18.0,15.2,-3.9,-4.6$; IR (neat) 2931, $2858,1740,1614,1513,1458,1375,1245,1090 \mathrm{~cm}^{-1}$; ESI/MS $(\mathrm{m} / \mathrm{z})$ calcd for $\mathrm{C}_{57} \mathrm{H}_{80} \mathrm{O}_{13} \mathrm{SiNa} 1023.5\left(\mathrm{M}+\mathrm{Na}^{+}\right)$, found 1023.5.

$(2 R, 4 a R, 5 a S, 6 a R, 7 a S, 8 a R, 9 a S, 11 R, 12 S, 13 a R, 15 a S, 16 a R, 17 a S, 18 a R, 19 a S)-12-(2-$ (benzyloxy)ethyl)-11-((4-methoxybenzyl)oxy)-4a,12,13a-trimethyl-2-phenylicosahydro-[1,3]dioxino [4"', 5"'":5"',6"']pyrano[2"',3"':5",6"]pyrano[2",3":5',6']pyrano[2',3':5,6]pyrano[3,2-b]pyrano[2,3-f]oxepin-7a(4H)-ol (19). To a solution of $18(17.7 \mathrm{mg}, 0.0185 \mathrm{mmol})$ in THF $(10 \mathrm{ml})$ at $0{ }^{\circ} \mathrm{C}, \mathrm{HF} \bullet P y(0.60 \mathrm{ml}, 33$ $\mathrm{mmol}$ ) was slowly added. The reaction mixture was allowed to warm to RT and stirred for 2 days before it was quenched with sat. $\mathrm{NaHCO}_{3}$ (aq., $50 \mathrm{ml}$ ). The aqueous phase was extracted with ethyl acetate $(3 \times 10 \mathrm{ml})$ and the combined organic phase was dried $\left(\mathrm{Na}_{2} \mathrm{SO}_{4}\right)$ and concentrated. Flash chromatography (2:1 hexanes:ethyl acetate) provided 19 as a colorless oil $(15.5 \mathrm{mg}, 99 \%) . R_{\mathrm{f}} 0.35$ (2:1 hexanes:ethyl acetate); $[\alpha]^{20}{ }_{\mathrm{D}}=-15.8^{\circ} \quad(c=0.22$, THF $) ;{ }^{1} \mathrm{H}$ NMR $\left(500 \mathrm{MHz}, \quad \mathrm{CD}_{2} \mathrm{Cl}_{2}\right) \quad \delta \quad 7.48-7.45(\mathrm{~m}, \quad 2 \mathrm{H}), \quad 7.39-7.24 \quad(\mathrm{~m}, \quad 8 \mathrm{H}), \quad 7.23$ $(\mathrm{d}, J=8.3 \mathrm{~Hz}, 2 \mathrm{H}), \quad 6.86 \quad(\mathrm{~d}, \quad J=8.3 \mathrm{~Hz}, 2 \mathrm{H}), \quad 5.56 \quad(\mathrm{~s}, \quad 1 \mathrm{H}), \quad 4.55$ $(\mathrm{d}, J=11.2 \mathrm{~Hz}, 1 \mathrm{H}), 4.45(\mathrm{~d}, J=11.7 \mathrm{~Hz}, 1 \mathrm{H}), 4.41(\mathrm{~d}, J=12.2 \mathrm{~Hz}, 1 \mathrm{H}), 4.36$ $(\mathrm{d}, J=10.7 \mathrm{~Hz}, 1 \mathrm{H}), 4.08($ br d, $J=6.8 \mathrm{~Hz}, 1 \mathrm{H}), 3.92(\mathrm{~d}, J=9.8 \mathrm{~Hz}, 1 \mathrm{H})$, 3.80-3.74 (m, $1 \mathrm{H}), 3.78(\mathrm{~s}, 3 \mathrm{H}), 3.68-3.62(\mathrm{~m}, 2 \mathrm{H}), 3.59-3.44(\mathrm{~m}, 4 \mathrm{H}), 3.42$ (partially obscured dd, $J=11.7,4.4 \mathrm{~Hz}, 1 \mathrm{H}), 3.33-3.27(\mathrm{~m}, 1 \mathrm{H}), 3.22$ (ddd, $J=11.7,2.4,1.5 \mathrm{~Hz}, 1 \mathrm{H}), 3.17$ (ddd, $J=12.7,9.3,3.4 \mathrm{~Hz}, 1 \mathrm{H}), 3.13$ (ddd, $J=11.2,9.3,3.9 \mathrm{~Hz}, 1 \mathrm{H}), 2.23-2.00(\mathrm{~m}, 4 \mathrm{H}), 1.93-1.88(\mathrm{~m}, 2 \mathrm{H}), 1.86-1.73$ $(\mathrm{m}, 3 \mathrm{H}), 1.73-1.65(\mathrm{~m}, 2 \mathrm{H}), 1.63-1.52(\mathrm{~m}, 2 \mathrm{H}), 1.50(\mathrm{~s}, 3 \mathrm{H}), 1.45$ (ddd, $J=11.7,11.7,11.7 \mathrm{~Hz}, 1 \mathrm{H}), 1.41-1.25(\mathrm{~m}, 2 \mathrm{H}), 1.22(\mathrm{~s}, 3 \mathrm{H}), 1.18$ (s, 3H), 1.16-1.95 (m, $1 \mathrm{H}) ;{ }^{13} \mathrm{C}$ NMR $\left(125 \mathrm{MHz}, \mathrm{CD}_{2} \mathrm{Cl}_{2}\right) \delta$ 138.0, 131.0, $129.3,129.2,128.4,127.8,127.5,126.5,113.8,103.0,93.9,82.2,81.9,79.8,79.6$, $79.3,78.6,77.6,77.0,76.3,75.6,72.9,70.8,70.2,68.9,66.5,55.4,49.2,43.2$, $41.8,39.7,35.3,34.1,30.6,30.2,29.2,28.8,25.8,25.2,22.4,22.2,15.0$; IR (neat) $3323,3055,2927,1624,1433,1265,1113 \mathrm{~cm}^{-1}$; ESI/MS $(\mathrm{m} / \mathrm{z})$ calcd for $\mathrm{C}_{49} \mathrm{H}_{62} \mathrm{O}_{12} \mathrm{Na} 865.4\left(\mathrm{M}+\mathrm{Na}^{+}\right)$, found 865.4.

(4aR,5aS,6aR,7aS,8aR,9aS,11R,12S,13aR,15aS,16aR,17aS,18aR,19aS)-12-(2(benzyloxy)ethyl)-4a,12,13a-trimethyldocosahydro-[1,3,2] dioxasilino[4"', $5^{\prime \prime \prime}: 5$ "', 6"']pyrano[2"',3"':5",6"]pyrano[2",3":5',6']pyrano[2',3':5,6]pyrano[3,2-b]pyrano [2,3-f]oxepin-11-ol (3). To a solution of $19(15.9 \mathrm{mg}, 0.0189 \mathrm{mmol})$ in $\mathrm{CH}_{2} \mathrm{Cl}_{2}(6 \mathrm{ml})$ and EtSH $(2 \mathrm{ml})$ was added $\mathrm{Zn}(\mathrm{OTf})_{2}(140 \mathrm{mg}, 0.385 \mathrm{mmol})$. The reaction mixture was heated to reflux and stirred for $12 \mathrm{~h}$ before it was cooled to RT and the reaction quenched with sat. $\mathrm{NaHCO}_{3}$ (aq., $5 \mathrm{ml}$ ). The aqueous phase was extracted with $\mathrm{CH}_{2} \mathrm{Cl}_{2}(3 \times 5 \mathrm{ml})$ and the combined organic phase was dried $\left(\mathrm{Na}_{2} \mathrm{SO}_{4}\right)$ and concentrated. Flash chromatography (20:1 dichloromethane:methanol) provided triol 20 as a colorless oil. 
To the solution of the triol obtained above in DMF $(10 \mathrm{ml})$ at $-20^{\circ} \mathrm{C}$ was added $t \mathrm{Bu}_{2} \mathrm{Si}(\mathrm{OTf})_{2}(14.0 \mu \mathrm{l}, 0.0384 \mathrm{mmol})$. The reaction mixture was stirred at $-20^{\circ} \mathrm{C}$ for $1 \mathrm{~h}$ before pyridine $(6.2 \mathrm{ml}, 0.077 \mathrm{mmol})$ was added. The mixture was stirred for another $5 \mathrm{~min}$ and the reaction was quenched with sat. $\mathrm{NaHCO}_{3}$ (aq., $10 \mathrm{ml})$. The aqueous phase was extracted with $\mathrm{Et}_{2} \mathrm{O}(3 \times 10 \mathrm{ml})$ and the combined organic phase was dried $\left(\mathrm{Na}_{2} \mathrm{SO}_{4}\right)$ and concentrated. Flash chromatography (3:1 hexanes:ethyl acetate) provided a colorless oil, which was taken on to the next transformation without additional purification.

To a solution of the colorless oil obtained above in toluene $(10 \mathrm{ml})$ was added $\mathrm{Ph}_{3} \mathrm{SnH}(200 \mathrm{mg}, 0.570 \mathrm{mmol})$. The reaction mixture was heated to reflux and AIBN (0.0046 $\mathrm{m}$ solution in toluene, $2.0 \mathrm{ml}$, $9.2 \mu \mathrm{mol})$ was added via syringe pump over $2 \mathrm{~h}$. The mixture was then cooled to RT and solvent was removed under reduced pressure. Flash chromatography (2:1 hexanes:ethyl acetate) provided 3 as a colorless oil $(7.3 \mathrm{mg}, 51 \% 3$ steps $) . R_{\mathrm{f}} 0.20(2: 1$ hexanes:ethyl acetate); $[\alpha]^{20}{ }_{\mathrm{D}}=-7.6^{\circ}\left(c=0.10\right.$, THF); ${ }^{1} \mathrm{H}$ NMR $(500 \mathrm{MHz}$, $\left.\mathrm{CD}_{2} \mathrm{Cl}_{2}\right) \delta 7.39-7.27(\mathrm{~m}, 5 \mathrm{H}), 4.50(\mathrm{~s}, 2 \mathrm{H}), 4.07(\mathrm{dd}, J=12.2,4.4 \mathrm{~Hz}, 1 \mathrm{H}), 3.88$ $(\mathrm{d}, J=10.2 \mathrm{~Hz}, 1 \mathrm{H}), 3.75(\mathrm{~d}, J=10.2 \mathrm{~Hz}, 1 \mathrm{H}), 3.59(\mathrm{dd}, J=6.4,4.4 \mathrm{~Hz}, 1 \mathrm{H})$, $3.54(\mathrm{dd}, J=12.2,3.9 \mathrm{~Hz}, 1 \mathrm{H}), 3.40-3.35(\mathrm{~m}, 1 \mathrm{H}), 3.33(\mathrm{dd}, J=11.7,3.9 \mathrm{~Hz}$, $1 \mathrm{H}), 3.26$ (ddd, $J=10.8,9.3,4.4 \mathrm{~Hz}, 1 \mathrm{H}$ ), 3.19 (ddd, $J=12.7,8.8,4.4 \mathrm{~Hz}, 1 \mathrm{H}$ ), $3.13-2.95(\mathrm{~m}, 6 \mathrm{H}), 2.32-2.24(\mathrm{~m}, 2 \mathrm{H}), 2.18-2.10(\mathrm{~m}, 2 \mathrm{H}), 2.00-1.68(\mathrm{~m}, 10 \mathrm{H})$, 1.65 (ddd, $J=11.7,11.7,11.7 \mathrm{~Hz}, 1 \mathrm{H}), 1.42(\mathrm{~s}, 3 \mathrm{H}), 1.41-1.34(\mathrm{~m}, 1 \mathrm{H}), 1.23$ (s, 3H), 1.16 (s, 3H), 1.10 (s, 9H), $1.03(\mathrm{~s}, 9 \mathrm{H}) ;{ }^{13} \mathrm{C}$ NMR $\left(125 \mathrm{MHz}, \mathrm{CD}_{2} \mathrm{Cl}_{2}\right)$ $\delta 138.4,129.0,128.4,128.4,81.9,81.4,80.4,78.6,77.8,77.7,77.6,77.4,76.8$, $75.0,74.9,74.3,73.7,73.2,69.8,67.1,43.8,40.5,37.4,35.9,35.7,34.0,32.3$, 29.9, 29.3, 28.0, 23.9, 20.4, 20.1, 15.6; IR (neat) $3417,2925,2855,1652,1457$, $1375,1080 \mathrm{~cm}^{-1} ; \mathrm{FAB} / \mathrm{MS}(\mathrm{m} / \mathrm{z})$ calcd for $\mathrm{C}_{42} \mathrm{H}_{66} \mathrm{O}_{10} \mathrm{SiAg} 865.3476\left(\mathrm{M}+\mathrm{Ag}^{+}\right)$, found 865.3475 .

(2R,4S,5R)-4-allyl-5-methyl-2-phenyl-1,3-dioxan-5-yl acetate (23). To a solution of $22(1.24 \mathrm{~g}, 5.30 \mathrm{mmol})$ in $\mathrm{CH}_{2} \mathrm{Cl}_{2}(25 \mathrm{ml})$ were added $\mathrm{Et}_{3} \mathrm{~N}(3.70 \mathrm{ml}$, $26.6 \mathrm{mmol}), \mathrm{Ac}_{2} \mathrm{O}(1.50 \mathrm{ml}, 15.6 \mathrm{mmol})$ and DMAP $(0.646 \mathrm{~g}, 5.30 \mathrm{mmol})$. The resulting mixture was stirred for $2 \mathrm{~h}$ before the reaction was quenched with sat. $\mathrm{NaHCO}_{3}$ (aq., $20 \mathrm{ml}$ ). The phases were separated and the aqueous phase was extracted with $\mathrm{CH}_{2} \mathrm{Cl}_{2}(3 \times 20 \mathrm{ml})$. The combined organic extracts were dried $\left(\mathrm{Na}_{2} \mathrm{SO}_{4}\right)$ and concentrated. Flash chromatography (5:1 hexanes:ethyl acetate) provided 23 as a colorless oil $(1.15 \mathrm{~g}, 79 \%) . R_{\mathrm{f}} 0.60$ (4:1 hexanes:ethyl acetate); $[\alpha]^{20}{ }_{\mathrm{D}}=-71.2^{\circ} \quad(c=0.32, \quad \mathrm{THF}) ;{ }^{1} \mathrm{H} \quad \mathrm{NMR} \quad\left(500 \mathrm{MHz}, \mathrm{C}_{6} \mathrm{D}_{6}\right) \quad \delta \quad 7.59$ $(\mathrm{d}, J=7.3 \mathrm{~Hz}, 2 \mathrm{H}), 7.18(\mathrm{t}, J=7.3 \mathrm{~Hz}, 2 \mathrm{H}), 7.12(\mathrm{t}, J=7.3 \mathrm{~Hz}, 1 \mathrm{H}), 5.96$ (dddd, $J=17.6,10.3,7.3,6.3 \mathrm{~Hz}, 1 \mathrm{H}), 5.34(\mathrm{~s}, 1 \mathrm{H}), 5.14(\mathrm{~d}, J=17.1 \mathrm{~Hz}, 1 \mathrm{H})$, $5.08(\mathrm{~d}, J=10.3 \mathrm{~Hz}, 1 \mathrm{H}), 4.99(\mathrm{~d}, J=10.7 \mathrm{~Hz}, 1 \mathrm{H}), 3.73(\mathrm{dd}, J=10.1,2.7 \mathrm{~Hz}$, $1 \mathrm{H}), 3.64(\mathrm{~d}, J=10.7 \mathrm{~Hz}, 1 \mathrm{H}), 2.40-2.33(\mathrm{~m}, 1 \mathrm{H}), 2.29-2.21(\mathrm{~m}, 1 \mathrm{H}), 1.69$ $(\mathrm{s}, 3 \mathrm{H}), 1.56(\mathrm{~s}, 3 \mathrm{H}) ;{ }^{13} \mathrm{C}$ NMR $\left(125 \mathrm{MHz}, \mathrm{C}_{6} \mathrm{D}_{6}\right) \delta 168.8,138.5,135.2,128.8$, $128.2,126.5,116.8,101.7,81.6,75.7,74.2,33.5,21.1,16.1$; IR (neat) 2985, 2859, 1737, 1643,1452, 1367, 1237, $1099 \mathrm{~cm}^{-1}$; ESI/MS $(\mathrm{m} / \mathrm{z})$ calcd for $\mathrm{C}_{16} \mathrm{H}_{20} \mathrm{O}_{4} \mathrm{Na} 299.1\left(\mathrm{M}+\mathrm{Na}^{+}\right)$, found 299.1.

(2R,4aR,8aS)-4a,6-dimethyl-2-phenyl-4,4a,8,8a-tetrahydropyrano[3,2-d][1,3] dioxine (24). To a solution of $\mathrm{TiCl}_{4}(7.37 \mathrm{ml}, 67.2 \mathrm{mmol})$ in $\mathrm{CH}_{2} \mathrm{Cl}_{2}(440 \mathrm{ml})$ at $0{ }^{\circ} \mathrm{C}$ was added THF $(35.5 \mathrm{ml}, 0.404 \mathrm{~mol})$ dropwise. To the resulting yellow solution, TMEDA $(60.9 \mathrm{ml}, 0.404 \mathrm{~mol})$ was added dropwise. The solution turned a red-brown color. The ice bath was removed and the mixture was allowed to stir for $15 \mathrm{~min}$ after which activated $\mathrm{Zn}$ dust $(9.83 \mathrm{~g}, 0.151 \mathrm{~mol})$ and $\mathrm{PbCl}_{2}(2.22 \mathrm{~g}, 7.98 \mathrm{mmol})$ were added. The resulting mixture went through a series of color changes from brown to green to purple and finally to blue-green over the course of $10 \mathrm{~min}$. To the resulting slurry was transferred a solution of ester $23(1.16 \mathrm{~g}, 4.20 \mathrm{mmol})$ and $\mathrm{CH}_{3} \mathrm{CHBr}_{2}(6.02 \mathrm{ml}, 67.2 \mathrm{mmol})$ in $\mathrm{CH}_{2} \mathrm{Cl}_{2}$ $(20 \mathrm{ml})$ via cannula. The reaction mixture was then heated to reflux for $2 \mathrm{~h}$ before it was cooled to $0{ }^{\circ} \mathrm{C}$. The reaction was quenched by adding sat. $\mathrm{K}_{2} \mathrm{CO}_{3}$ (aq., $50 \mathrm{ml}$ ). The resulting mixture was stirred for $30 \mathrm{~min}$, filtered and the filtrate was concentrated. Flash chromatography (15:1 hexanes:ethyl acetate) gave 24 as a colorless oil $(0.949 \mathrm{~g}, 92 \%) . R_{\mathrm{f}} 0.70$ (5:1 hexanes:ethyl acetate); $[\alpha]^{20}{ }_{\mathrm{D}}=+23.0^{\circ} \quad(c=0.43, \quad \mathrm{THF}) ; \quad{ }^{1} \mathrm{H} \quad \mathrm{NMR} \quad\left(500 \mathrm{MHz}, \mathrm{C}_{6} \mathrm{D}_{6}\right) \quad \delta \quad 7.52$ $(\mathrm{d}, J=7.3 \mathrm{~Hz}, 2 \mathrm{H}), 7.17(\mathrm{t}, J=7.3 \mathrm{~Hz}, 2 \mathrm{H}), 7.12(\mathrm{t}, J=7.3 \mathrm{~Hz}, 1 \mathrm{H}), 5.37$ $(\mathrm{s}, 1 \mathrm{H}), 4.22(\mathrm{~d}, J=4.4 \mathrm{~Hz}, 1 \mathrm{H}), 3.94(\mathrm{~d}, J=9.8 \mathrm{~Hz}, 1 \mathrm{H}), 3.65(\mathrm{dd}, J=10.7$, $6.4 \mathrm{~Hz}, 1 \mathrm{H}), 3.60(\mathrm{~d}, J=10.3 \mathrm{~Hz}, 1 \mathrm{H}), 2.12-1.98(\mathrm{~m}, 2 \mathrm{H}), 1.61(\mathrm{~s}, 3 \mathrm{H})$, $1.34(\mathrm{~s}, 3 \mathrm{H}) ;{ }^{13} \mathrm{C}$ NMR $\left(125 \mathrm{MHz}, \mathrm{C}_{6} \mathrm{D}_{6}\right) \delta 148.5,138.5,128.8,128.1,126.6$, 102.3, 92.9, 77.1, 75.7, 69.6, 24.0, 19.9, 15.3; IR (neat) 2921, 2859, 1673, 1453,
1378, 1312, 1150, $1093 \mathrm{~cm}^{-1}$; ESI/MS $(\mathrm{m} / z)$ calcd for $\mathrm{C}_{15} \mathrm{H}_{18} \mathrm{O}_{3} \mathrm{Na} 269.1$ $\left(\mathrm{M}+\mathrm{Na}^{+}\right)$, found 269.1 .

$(2 R, 4 a R, 6 S, 8 a S)-6$-allyl-4a,6-dimethyl-2-phenyltetrahydropyrano[3,2-d][1,3] dioxin-7(6H)-one (26). To a solution of $24(0.576 \mathrm{~g}, 2.34 \mathrm{mmol})$ in $\mathrm{MeOH}$ $(30 \mathrm{ml})$ at $-78^{\circ} \mathrm{C}$ was added $m \mathrm{CPBA}(77 \%, 1.57 \mathrm{~g}, 7.00 \mathrm{mmol})$. The reaction mixture was slowly warmed up to $0{ }^{\circ} \mathrm{C}$, at which point sat. $\mathrm{NaHCO}_{3}$ (aq., $30 \mathrm{ml})$ was added. The mixure was extracted with $\mathrm{CH}_{2} \mathrm{Cl}_{2}(3 \times 15 \mathrm{ml})$ and the combined organic extracts were dried $\left(\mathrm{Na}_{2} \mathrm{SO}_{4}\right)$ and concentrated. Flash chromatography (3:1 hexanes:ethyl acetate) provided a white solid $(0.550 \mathrm{~g}, 80 \%)$ which was used in the next transformaiton without additional purification.

To a solution of the material obtained above in THF $(30 \mathrm{ml})$ were added $\mathrm{NaH}(88.0 \mathrm{mg}, 3.67 \mathrm{mmol})$, allyl bromide $(0.800 \mathrm{ml}, 9.19 \mathrm{mmol})$ and TBAI $(50.0 \mathrm{mg}, 0.135 \mathrm{mmol})$. The resulting reaction mixture was heated to reflux overnight. After cooling to RT, the reaction was quenched with sat. $\mathrm{NH}_{4} \mathrm{Cl}$ (aq., $20 \mathrm{ml})$. The aqueous phase was extracted with $\mathrm{CH}_{2} \mathrm{Cl}_{2}(3 \times 15 \mathrm{ml})$ and the organic extracts were combined, dried $\left(\mathrm{Na}_{2} \mathrm{SO}_{4}\right)$ and concentrated. The resulting residue was passed through a short silica gel plug (5:1 hexanes:ethyl acetate) and concentrated to give a colorless oil $\mathbf{2 5}$, which was taken into the subsequent transformation without additional purification.

To the colorless oil obtained above in toluene $(50 \mathrm{ml})$ was added pyridine $(7.40 \mathrm{ml}, 91.6 \mathrm{mmol})$ and PPTS $(2.76 \mathrm{~g}, 11.0 \mathrm{mmol})$. The resulting reaction mixture was heated to reflux for $16 \mathrm{~h}$. The mixture was cooled to RT and the reaction was quenched with sat. $\mathrm{NaHCO}_{3}$ (aq., $30 \mathrm{ml}$ ). The aqueous phase was extracted with $\mathrm{CH}_{2} \mathrm{Cl}_{2}(3 \times 20 \mathrm{ml})$ and the combined organic extracts were dried $\left(\mathrm{Na}_{2} \mathrm{SO}_{4}\right)$ and concentrated. Flash chromatography (5:1 hexanes:ethyl acetate) provided $26\left(0.409 \mathrm{~g}, 72 \%\right.$ two steps). $R_{\mathrm{f}} 0.70$ (3:1 hexanes:ethyl acetate); $[\alpha]^{20}{ }_{\mathrm{D}}=+11.2^{\circ}\left(c=0.43\right.$, THF); ${ }^{1} \mathrm{H}$ NMR $\left(500 \mathrm{MHz}, \mathrm{C}_{6} \mathrm{D}_{6}\right) \delta 7.50(\mathrm{~d}$, $J=7.3 \mathrm{~Hz}, 2 \mathrm{H}), 7.20(\mathrm{t}, J=7.3 \mathrm{~Hz}, 2 \mathrm{H}), 7.14(\mathrm{t}, J=7.3 \mathrm{~Hz}, 1 \mathrm{H}), 5.77$ (dddd, $J=17.6,10.3,7.3,7.3 \mathrm{~Hz}, 1 \mathrm{H}), 5.31(\mathrm{~s}, 1 \mathrm{H}), 5.03(\mathrm{~d}, J=10.3 \mathrm{~Hz}, 1 \mathrm{H}), 4.99(\mathrm{~d}$, $J=17.6 \mathrm{~Hz}, 1 \mathrm{H}), 3.85(\mathrm{~d}, J=10.3 \mathrm{~Hz}, 1 \mathrm{H}), 3.81(\mathrm{dd}, J=12.2,7.0 \mathrm{~Hz}, 1 \mathrm{H}), 3.47$ $(\mathrm{d}, J=10.3 \mathrm{~Hz}, 1 \mathrm{H}), 2.61(\mathrm{dd}, J=18.6,6.8 \mathrm{~Hz}, 1 \mathrm{H}), 2.41(\mathrm{dd}, J=18.6,11.7 \mathrm{~Hz}$, $1 \mathrm{H}), 2.30(\mathrm{dd}, J=13.2,7.5 \mathrm{~Hz}, 1 \mathrm{H}), 2.14(\mathrm{dd}, J=13.7,7.5 \mathrm{~Hz}, 1 \mathrm{H}), 1.25(\mathrm{~s}$, $3 \mathrm{H}), 1.19(\mathrm{~s}, 3 \mathrm{H}) ;{ }^{13} \mathrm{C}$ NMR $\left(100 \mathrm{MHz}, \mathrm{C}_{6} \mathrm{D}_{6}\right) \delta 208.8,138.1,133.0,128.9$, $128.2,126.5,119.1,101.8,83.8,76.3,68.3,46.4,39.4,26.5,17.1$; IR (neat) 2982 , $2865,1716,1521,1456,1372,1143,1114 \mathrm{~cm}^{-1}$; ESI/MS $(\mathrm{m} / \mathrm{z})$ calcd for $\mathrm{C}_{18} \mathrm{H}_{22} \mathrm{O}_{4} \mathrm{Na} 325.2\left(\mathrm{M}+\mathrm{Na}^{+}\right)$, found 325.1.

Summary of COSY spectrum for 26

1. Proton at 5.77 p.p.m. (C-25) shows cross peaks with protons at 5.03 p.p.m. (C-26), 4.99 p.p.m. (C-26), 2.30 p.p.m. (C-24) and 2.14 (C-24').

2. Proton at 3.85 p.p.m. (C-18) shows cross peaks with proton at 3.47 p.p.m. (C-18).

3. Proton at 3.81 p.p.m. (C-20) shows cross peaks with protons at 2.61 p.p.m. (C-21) and 2.41 p.p.m. (C-21).

4. Proton at 2.61 p.p.m. (C-21) shows cross peaks with proton at 2.41 p.p.m. (C-21).

5. Proton at 2.30 p.p.m. (C-24) shows cross peaks with proton at 2.14 p.p.m. (C-24).

Summary of $1 \mathrm{D}$ nOe spectrum for $\mathbf{2 6}$

1. Irradiation at 1.25 p.p.m. (C-23 methyl) resulted in enhancement at 1.19 p.p.m. (C-19 methyl) and 2.30 p.p.m. (C-24) and 2.14 p.p.m. (C-24').

(2R,4aR,6S,7R,8aS)-6-allyl-7-((4-methoxybenzyl)oxy)-4a,6-dimethyl-2-phenylhexahydropyrano[3,2-d][1,3]dioxine (27). To a solution of 26 (378 mg, $1.25 \mathrm{mmol})$ in $\mathrm{MeOH}(25 \mathrm{ml})$ at $0{ }^{\circ} \mathrm{C}$ was added $\mathrm{NaBH}_{4}(190 \mathrm{mg}, 5.0 \mathrm{mmol})$. The reaction was quenched with acetone $(10 \mathrm{ml})$ after the reaction mixture had been stirred for $2 \mathrm{~h}$. The solvent was then removed under reduced pressure and the residue was purified using flash chromatography (3:1 hexanes:ethyl acetate) to give the corresponding alcohol as a colorless oil (382 mg, 100\%).

To a solution of the alcohol obtained above $(382 \mathrm{mg}, 1.25 \mathrm{mmol})$ in THF $(20 \mathrm{ml})$ at $0{ }^{\circ} \mathrm{C}$ were added $\mathrm{NaH}(82.4 \mathrm{mg}, 3.43 \mathrm{mmol}), \operatorname{PMBBr}(0.82 \mathrm{ml}$, $5.69 \mathrm{mmol})$, HMPA $(0.220 \mathrm{ml}, 1.15 \mathrm{mmol})$ and TBAI $(50 \mathrm{mg}, 0.135 \mathrm{mmol})$. The reaction mixture was stirred at RT for $12 \mathrm{~h}$ before the reaction was 
quenched with sat. $\mathrm{NH}_{4} \mathrm{Cl}$ (aq., $20 \mathrm{ml}$ ). The mixure was extracted with $\mathrm{CH}_{2} \mathrm{Cl}_{2}$ $(3 \times 15 \mathrm{ml})$ and the combined organic extracts were dried $\left(\mathrm{Na}_{2} \mathrm{SO}_{4}\right)$ and concentrated. Flash chromatography (10:1 hexanes:ethyl acetate) gave 27 as a colorless oil (442 mg, 83\%). $R_{\mathrm{f}} 0.55$ (5:1 hexanes:ethyl acetate); $[\alpha]^{20}{ }_{\mathrm{D}}=-32.8^{\circ}$ $(c=0.30, \mathrm{THF}) ;{ }^{1} \mathrm{H}$ NMR $\left(500 \mathrm{MHz}, \mathrm{C}_{6} \mathrm{D}_{6}\right) \delta 7.64(\mathrm{~d}, J=7.8 \mathrm{~Hz}, 2 \mathrm{H})$, 7.27-7.12 (m, 5H), $6.82(\mathrm{~d}, J=7.8 \mathrm{~Hz}, 2 \mathrm{H}), 6.07$ (dddd, $J=17.1,9.8,7.3$, $7.3 \mathrm{~Hz}, 1 \mathrm{H}), 5.39$ (s, 1H), 5.14 (dd, $J=9.8,1.3 \mathrm{~Hz}, 1 \mathrm{H}), 5.04$ (dd, $J=17.6$, $1.0 \mathrm{~Hz}, 1 \mathrm{H}), 4.44(\mathrm{~d}, J=11.2 \mathrm{~Hz}, 1 \mathrm{H}), 4.24(\mathrm{~d}, J=11.7 \mathrm{~Hz}, 1 \mathrm{H}), 3.85$ $(\mathrm{d}, J=9.7 \mathrm{~Hz}, 1 \mathrm{H}), 3.44(\mathrm{dd}, J=11.2,4.4 \mathrm{~Hz}, 1 \mathrm{H}), 3.40(\mathrm{~d}, J=9.7 \mathrm{~Hz}, 1 \mathrm{H})$, $3.32(\mathrm{~s}, 3 \mathrm{H}), 3.23(\mathrm{dd}, J=12.7,3.4 \mathrm{~Hz}, 1 \mathrm{H}), 2.46(\mathrm{dd}, J=13.7,6.4 \mathrm{~Hz}, 1 \mathrm{H})$, $2.33(\mathrm{dd}, J=14.2,7.8 \mathrm{~Hz}, 1 \mathrm{H}), 2.18$ (ddd, $J=11.7,3.9,3.9 \mathrm{~Hz}, 1 \mathrm{H}), 1.85$ (ddd, $J=11.7,11.7,11.7 \mathrm{~Hz}, 1 \mathrm{H}), 1.53(\mathrm{~s}, 3 \mathrm{H}), 1.34(\mathrm{~s}, 3 \mathrm{H}) ;{ }^{13} \mathrm{C}$ NMR $(125 \mathrm{MHz}$, $\left.\mathrm{C}_{6} \mathrm{D}_{6}\right) \delta 159.7,138.7,135.0,130.8,129.3,129.3,128.8,128.2,126.7,117.4$, 114.0, 102.7, 80.6, 78.6, 77.7, 76.9, 71.5, 70.3, 68.9, 54.7, 46.6, 26.8, 23.3, 19.1; IR (neat) 2947, 2864, 1611, 1512, 1461, 1376, 1247, 1144, $1086 \mathrm{~cm}^{-1}$; ESI/MS $(\mathrm{m} / \mathrm{z})$ calcd for $\mathrm{C}_{26} \mathrm{H}_{32} \mathrm{O}_{5} \mathrm{Na} 447.2\left(\mathrm{M}+\mathrm{Na}^{+}\right)$, found 447.2.

$(2 R, 4 a R, 6 S, 7 R, 8 a S)-6-(b u t-3-e n-1-y l)-7-((4-m e t h o x y b e n z y l) o x y)-4 a, 6-$ dimethyl2-phenylhexahydropyrano[3,2-d][1,3]dioxine (28). To a solution of 27 $(0.245 \mathrm{~g}, 0.578 \mathrm{mmol})$ in THF $(15 \mathrm{ml})$ at $0{ }^{\circ} \mathrm{C}$ was added $\mathrm{BH}_{3} \bullet \mathrm{DMS}$ $(0.87 \mathrm{ml}$ of $2.0 \mathrm{M}$ solution in THF, $1.7 \mathrm{mmol})$. After stirring for $2 \mathrm{~h} \mathrm{H}_{2} \mathrm{O}$ $(1.0 \mathrm{ml}), \mathrm{NaOH}\left(2.0 \mathrm{ml}\right.$ of $3.0 \mathrm{M}$ aq. solution), and $\mathrm{H}_{2} \mathrm{O}_{2}(5.0 \mathrm{ml}$ of $30 \%$ aq. solution) were added to the reaction mixture. The mixture was warmed to RT and stirred overnight. The aqueous phase was extracted with ethyl acetate $(3 \times 10 \mathrm{ml})$. The combined organic extracts were dried $\left(\mathrm{Na}_{2} \mathrm{SO}_{4}\right)$ and concentrated.

To the product obtained above in $\mathrm{CH}_{2} \mathrm{Cl}_{2}(15 \mathrm{ml})$ were added $4 \AA$ MS $(0.60 \mathrm{~g}), \mathrm{NMO}(0.680 \mathrm{~g}, 5.78 \mathrm{mmol})$, and TPAP $(5 \mathrm{mg}, 14.2 \mu \mathrm{mol})$. The resulting mixture was stirred at RT for $2 \mathrm{~h}$ and then concentrated. The residue was filtered through a short silica gel plug (5:1 hexanes:ethyl acetate) to give the crude aldehyde.

To a slurry of methyltriphenylphosphonium bromide (1.03 g, $2.88 \mathrm{mmol})$ in THF $(10 \mathrm{ml})$ was added $t$ BuOK $(2.9 \mathrm{ml}$ of $1.0 \mathrm{M}$ solution in THF, $2.9 \mathrm{mmol})$. After stirring at RT for $30 \mathrm{~min}$, the resulting solution was transferred to a solution of the crude aldehyde from above in THF $(10 \mathrm{ml})$. After $2 \mathrm{~h}$ the reaction was quenched with $\mathrm{NH}_{4} \mathrm{Cl}$ (aq., $10 \mathrm{ml}$ ). The aqueous phase was extracted with $\mathrm{CH}_{2} \mathrm{Cl}_{2}(3 \times 10 \mathrm{ml})$. The organic extracts were combined, dried $\left(\mathrm{Na}_{2} \mathrm{SO}_{4}\right)$ and concentrated. Flash chromatography (10:1 hexanes:ethyl acetate) gave 28 as a colorless oil $\left(0.154 \mathrm{~g}, 61 \%\right.$ for 3 steps). $R_{\mathrm{f}} 0.60$ (5:1 hexanes:ethyl acetate); $[\alpha]^{20}{ }_{\mathrm{D}}=-55.4^{\circ}(c=0.37, \mathrm{THF}) ;{ }^{1} \mathrm{H}$ NMR $\left(500 \mathrm{MHz}, \mathrm{C}_{6} \mathrm{D}_{6}\right) \delta 7.64$ $(\mathrm{d}, J=7.3 \mathrm{~Hz}, 2 \mathrm{H}), 7.22(\mathrm{t}, J=7.3 \mathrm{~Hz}, 2 \mathrm{H}), 7.18-7.12(\mathrm{~m}, 3 \mathrm{H}), 6.81$ (d, $J=8.3 \mathrm{~Hz}, 2 \mathrm{H}), 5.85$ (dddd, $J=17.1,10.2,6.8,6.3 \mathrm{~Hz}, 1 \mathrm{H}), 5.42(\mathrm{~s}, 1 \mathrm{H})$, $5.09(\mathrm{dd}, J=17.1,1.5 \mathrm{~Hz}, 1 \mathrm{H}), 5.00(\mathrm{dd}, J=10.2,1.5 \mathrm{~Hz}, 1 \mathrm{H}), 4.44$ $(\mathrm{d}, J=11.2 \mathrm{~Hz}, 1 \mathrm{H}), 4.20(\mathrm{~d}, J=11.7 \mathrm{~Hz}, 1 \mathrm{H}), 3.84(\mathrm{~d}, J=9.8 \mathrm{~Hz}, 1 \mathrm{H}), 3.39$ (d, $J=10.8 \mathrm{~Hz}, 1 \mathrm{H}), 3.36$ (dd, $J=11.2,4.9 \mathrm{~Hz}, 1 \mathrm{H}), 3.31(\mathrm{~s}, 3 \mathrm{H}), 3.24$ (dd, $J=12.2,3.4 \mathrm{~Hz}, 1 \mathrm{H}), 2.24-2.12(\mathrm{~m}, 3 \mathrm{H}), 1.86(\mathrm{ddd}, J=11.7,11.7,11.7 \mathrm{~Hz}$, $1 \mathrm{H}), 1.81$ (ddd, $J=13.2,11.2,5.4 \mathrm{~Hz}, 1 \mathrm{H}), 1.64$ (ddd, $J=13.7,11.2,5.4 \mathrm{~Hz}$, $1 \mathrm{H}), 1.53(\mathrm{~s}, 3 \mathrm{H}), 1.33(\mathrm{~s}, 3 \mathrm{H}) ;{ }^{13} \mathrm{C}$ NMR $\left(125 \mathrm{MHz}, \mathrm{C}_{6} \mathrm{D}_{6}\right) \delta 160.2,140.0$, 139.2, 131.1, 129.9, 129.3, 128.7, 127.2, 114.6, 114.5, 103.2, 81.3, 79.5, 77.9, $77.5,70.7,69.3,55.2,42.1,28.3,27.1,23.5,19.6$; IR (neat) 2943, 2863, 1639, $1513,1460,1375,1248,1086 \mathrm{~cm}^{-1}$; ESI/MS $(\mathrm{m} / z)$ calcd for $\mathrm{C}_{27} \mathrm{H}_{34} \mathrm{O}_{5} \mathrm{~K} 477.2$ $\left(\mathrm{M}+\mathrm{K}^{+}\right)$, found 477.2.

$(2 R, 4 a R, 6 S, 7 R, 8 a S)-7-((4-m e t h o x y b e n z y l) o x y)-4 a, 6$-dimethyl-6-(pent-4-en-1-yl)2-phenylhexahydropyrano[3,2-d][1,3]dioxine (29). To a solution of $\mathbf{2 8}$ $(74.6 \mathrm{mg}, 0.170 \mathrm{mmol})$ in THF $(5 \mathrm{ml})$ at $0{ }^{\circ} \mathrm{C}$ was added $\mathrm{BH}_{3} \bullet \mathrm{DMS}$ $(0.340 \mathrm{ml}$ of $2.0 \mathrm{M}$ solution in THF, $0.680 \mathrm{mmol})$. The reaction mixture was stirred for 2 before it was quenched with $\mathrm{H}_{2} \mathrm{O}(1.0 \mathrm{ml}), \mathrm{NaOH}(1.0 \mathrm{ml}$ of $3.0 \mathrm{M}$ aq. solution), and $\mathrm{H}_{2} \mathrm{O}_{2}(3.0 \mathrm{ml}$ of $30 \%$ aq. solution). The reaction mixture was then warmed to RT and stirred overnight. The aqueous phase was extracted with ethyl acetate $(3 \times 10 \mathrm{ml})$. The combined organic phase was dried $\left(\mathrm{Na}_{2} \mathrm{SO}_{4}\right)$ and concentrated.

To the crude product obtained from above in $\mathrm{CH}_{2} \mathrm{Cl}_{2}(10 \mathrm{ml})$ was added $4 \AA \mathrm{MS}(0.20 \mathrm{~g}), \mathrm{NMO}(0.200 \mathrm{~g}, 1.71 \mathrm{mmol})$, and TPAP $(2.0 \mathrm{mg}, 5.7 \mu \mathrm{mol})$. After stirring for $2 \mathrm{~h}$ the reaction mixture was concentrated. The resulting residue was filtered through a short silica gel plug (5:1 hexanes:ethyl acetate) to give the crude aldehyde.
To a slurry of methyltriphenylphosphonium bromide ( $300 \mathrm{mg}, 0.840 \mathrm{mmol}$ ) in THF $(5 \mathrm{ml})$ was added $t \mathrm{BuOK}(0.84 \mathrm{ml}$ of $1.0 \mathrm{~m}$ solution in THF, $0.84 \mathrm{mmol}$ ). After stirring at RT for $30 \mathrm{~min}$, the solution was transferred to a solution of the crude aldehyde from above in THF $(5 \mathrm{ml})$. The reaction mixture was stirred for an additional $2 \mathrm{~h}$ before the reaction was quenched with $\mathrm{NH}_{4} \mathrm{Cl}$ (aq., $10 \mathrm{ml})$. The aqueous phase was extracted with $\mathrm{CH}_{2} \mathrm{Cl}_{2}(3 \times 10 \mathrm{ml})$. The organic extracts were combined, dried $\left(\mathrm{Na}_{2} \mathrm{SO}_{4}\right)$ and concentrated. Flash chromatography (15:1 hexanes:ethyl acetate) gave 29 as a colorless oil (42.1 mg, $55 \%$ for three steps). $R_{\mathrm{f}} 0.60$ (5:1 hexanes:ethyl acetate); $[\alpha]^{20}{ }_{\mathrm{D}}=-24.2^{\circ}$ $(c=0.24, \mathrm{THF}) ;{ }^{1} \mathrm{H}$ NMR $\left(500 \mathrm{MHz}, \mathrm{C}_{6} \mathrm{D}_{6}\right) \delta 7.66(\mathrm{~d}, J=7.3 \mathrm{~Hz}, 2 \mathrm{H}), 7.22$ (t, $J=7.3 \mathrm{~Hz}, 2 \mathrm{H}), 7.20-7.12(\mathrm{~m}, 3 \mathrm{H}), 6.82(\mathrm{~d}, J=8.8 \mathrm{~Hz}, 2 \mathrm{H}), 5.83$ (dddd, $J=16.6,9.8,6.3,6.3 \mathrm{~Hz}, 1 \mathrm{H}), 5.41(\mathrm{~s}, 1 \mathrm{H}), 5.08(\mathrm{dd}, J=17.1,1.5 \mathrm{~Hz}, 1 \mathrm{H}), 5.02$ (d, $J=10.2 \mathrm{~Hz}, 1 \mathrm{H}), 4.46(\mathrm{~d}, J=11.7 \mathrm{~Hz}, 1 \mathrm{H}), 4.20(\mathrm{~d}, J=11.7 \mathrm{~Hz}, 1 \mathrm{H}), 3.85$ (d, $J=9.8 \mathrm{~Hz}, 1 \mathrm{H}), 3.40(\mathrm{~d}, J=10.3 \mathrm{~Hz}, 1 \mathrm{H}), 3.37$ (dd, $J=11.2,4.9 \mathrm{~Hz}, 1 \mathrm{H})$, $3.31(\mathrm{~s}, 3 \mathrm{H}), 3.25(\mathrm{dd}, J=12.2,3.4 \mathrm{~Hz}, 1 \mathrm{H}), 2.20(\mathrm{ddd}, J=9.7,3.9,3.9 \mathrm{~Hz}, 1 \mathrm{H})$, 2.02-1.96 (m, 3H), 1.88 (ddd, $J=11.7,11.7,11.7 \mathrm{~Hz}, 1 \mathrm{H}), 1.73-1.66(\mathrm{~m}, 1 \mathrm{H})$, $1.55(\mathrm{~s}, 3 \mathrm{H}), 1.55-1.45(\mathrm{~m}, 3 \mathrm{H}), 1.35(\mathrm{~s}, 3 \mathrm{H}) ;{ }^{13} \mathrm{C} \mathrm{NMR}\left(125 \mathrm{MHz}, \mathrm{C}_{6} \mathrm{D}_{6}\right) \delta$ 159.7, 139.1, 138.7, 130.7, 129.5, 128.9, 128.2, 126.7, 114.5, 114.0, 102.7, 80.8, 78.9, 77.7, 77.1, 70.2, 68.8, 54.7, 42.0, 34.6, 26.6, 23.1, 22.6, 19.2; IR (neat) $2943,2864,1639,1513,1461,1375,1248,1086 \mathrm{~cm}^{-1}$; ESI/MS $(\mathrm{m} / \mathrm{z})$ calcd for $\mathrm{C}_{28} \mathrm{H}_{36} \mathrm{O}_{5} \mathrm{Na} 475.3\left(\mathrm{M}+\mathrm{Na}^{+}\right)$, found 475.2.

$(2 R, 4 a R, 6 S, 7 R, 8 a S)-2-(4-m e t h o x y p h e n y l)-4 a, 6$-dimethyl-6-(pent-4-en-1-yl) hexahydropyrano[3,2-d][1,3]dioxin-7-ol (30). To a solution of 29 (42.1 mg, $93.1 \mu \mathrm{mol})$ in $\mathrm{MeOH}(5 \mathrm{ml})$ at $0{ }^{\circ} \mathrm{C}$ was added CSA $(6.5 \mathrm{mg}, 28 \mu \mathrm{mol})$. The reaction mixture was warmed to RT and stirred for $5 \mathrm{~h}$ before the reaction was quenched with sat. $\mathrm{NaHCO}_{3}$ (aq., $5 \mathrm{ml}$ ). The aqueous phase was extracted with ethyl acetate $(3 \times 5 \mathrm{ml})$. The combined organic extracts were dried $\left(\mathrm{Na}_{2} \mathrm{SO}_{4}\right)$, and concentrated. Flash chromatography (3:1 hexanes:ethyl acetate) gave the corresponding diol as a colorless oil $(29.3 \mathrm{mg}, 86 \%)$.

To a solution of the diol obtained above in $\mathrm{CH}_{2} \mathrm{Cl}_{2}(4.5 \mathrm{ml})$ was added $\mathrm{pH} 7$ buffer $(0.5 \mathrm{ml})$ and DDQ $(36.5 \mathrm{mg}, 0.161 \mathrm{mmol})$. The reaction mixture was stirred at RT for $2 \mathrm{~h}$ before $\mathrm{H}_{2} \mathrm{O}(5 \mathrm{ml})$ was added. The aqueous phase was extracted with ethyl acetate $(3 \times 5 \mathrm{ml})$ and the combined organic extracts were dried $\left(\mathrm{Na}_{2} \mathrm{SO}_{4}\right)$, and concentrated. Flash chromatography (2:1 hexanes:ethyl acetate) gave the corresponding triol as a colorless oil (18.9 mg, 96\%).

To a solution of the triol obtained from the procedure outlined in the previous paragraph in $\mathrm{CH}_{2} \mathrm{Cl}_{2}(5 \mathrm{ml})$ was added anisaldehyde dimethylacetal $(19.8 \mu \mathrm{L}, 0.116 \mathrm{mmol})$ and CSA $(9.0 \mathrm{mg}, 39 \mu \mathrm{mol})$. The reaction mixture was stirred at RT overnight before the reaction was quenched with $\mathrm{NaHCO}_{3}$ (aq., $5 \mathrm{ml})$. The aqueous phase was extracted with ethyl acetate $(3 \times 5 \mathrm{ml})$ and the combined organic extracts were dried $\left(\mathrm{Na}_{2} \mathrm{SO}_{4}\right)$, and concentrated. Flash chromatography (3:1 hexanes:ethyl acetate) gave $\mathbf{3 0}$ as a colorless oil $(24.8 \mathrm{mg}$, $88 \%) . R_{\mathrm{f}} 0.25$ (3:1 hexanes:ethyl acetate); $[\alpha]^{20}{ }_{\mathrm{D}}=-9.3^{\circ}\left(c=0.15\right.$, THF); ${ }^{1} \mathrm{H}$ NMR $\left(500 \mathrm{MHz}, \mathrm{C}_{6} \mathrm{D}_{6}\right) \delta 7.57(\mathrm{~d}, J=8.3 \mathrm{~Hz}, 2 \mathrm{H}), 6.83(\mathrm{~d}, J=8.3 \mathrm{~Hz}, 2 \mathrm{H})$, 5.84 (dddd, $J=17.1,10.3,6.8,6.8 \mathrm{~Hz}, 1 \mathrm{H}), 5.42(\mathrm{~s}, 1 \mathrm{H}), 5.08(\mathrm{~d}, J=17.6 \mathrm{~Hz}$, $1 \mathrm{H}), 5.02(\mathrm{~d}, J=10.3 \mathrm{~Hz}, 1 \mathrm{H}), 3.86(\mathrm{~d}, J=9.8 \mathrm{~Hz}, 1 \mathrm{H}), 3.42(\mathrm{~d}, J=9.8 \mathrm{~Hz}, 1 \mathrm{H})$, 3.38 (dd, $J=12.2,4.9 \mathrm{~Hz}, 1 \mathrm{H}$ ), 3.30-3.26 (partially obscured m, $1 \mathrm{H}$ ), 3.26 (s, $3 \mathrm{H}), 2.05-2.00(\mathrm{~m}, 2 \mathrm{H}), 1.87$ (ddd, $J=11.2,4.4,4.4 \mathrm{~Hz}, 1 \mathrm{H}), 1.80$ (ddd, $J=11.7,11.7,11.7 \mathrm{~Hz}, 1 \mathrm{H}), 1.64-1.58(\mathrm{~m}, 2 \mathrm{H}), 1.51(\mathrm{~s}, 3 \mathrm{H}), 1.53-1.41(\mathrm{~m}, 2 \mathrm{H})$, 1.15 (s, 3H); ${ }^{13} \mathrm{C}$ NMR $\left(125 \mathrm{MHz}, \mathrm{C}_{6} \mathrm{D}_{6}\right) \delta 160.9,139.6,131.6,128.5,115.1$, $114.2,103.3,81.3,78.6,77.5,73.7,69.4,55.1,42.6,35.1,31.4,23.1,22.2,19.6$; IR (neat) $3483,2943,2867,1615,1517,1465,1377,1250,1069 \mathrm{~cm}^{-1}$; ESI/MS $(m / z)$ calcd for $\mathrm{C}_{21} \mathrm{H}_{30} \mathrm{O}_{5} \mathrm{Na} 385.2\left(\mathrm{M}+\mathrm{Na}^{+}\right)$, found 385.2 .

(4aR,6S,7S,8aR)-6-allyl-7-hydroxy-7-methyltetrahydropyrano[3,2-d][1,3,2] dioxasilin-8(4H)-one (32). To a solution of $(\mathrm{COCl})_{2}(0.53 \mathrm{ml}, 6.2 \mathrm{mmol})$ in $\mathrm{CH}_{2} \mathrm{Cl}_{2}(40 \mathrm{ml})$ at $-78^{\circ} \mathrm{C}$ was added DMSO $(0.58 \mathrm{ml}, 8.2 \mathrm{mmol})$ dropwise. To this was added a solution of $31(1.90 \mathrm{~g}, 4.08 \mathrm{mmol})$ in $\mathrm{CH}_{2} \mathrm{Cl}_{2}(10 \mathrm{ml})$ via cannula. After stirring at $-78{ }^{\circ} \mathrm{C}$ for $45 \mathrm{~min}, \mathrm{Et}_{3} \mathrm{~N}(2.28 \mathrm{ml}, 16.4 \mathrm{mmol})$ was added and the reaction mixture was stirred for another $5 \mathrm{~min}$. The reaction was quenched with sat. $\mathrm{NH}_{4} \mathrm{Cl}$ (aq., $50 \mathrm{ml}$ ) and allowed to warm to RT. The aqueous phase was extracted with $\mathrm{CH}_{2} \mathrm{Cl}_{2}(3 \times 50 \mathrm{ml})$ and the combined organic extracts were dried $\left(\mathrm{Na}_{2} \mathrm{SO}_{4}\right)$ and concentrated. Flash chromatography (20:1 hexanes:ethyl acetate) provided the corresponding ketone (1.85 g, 98\%) as a colorless oil. 
To a solution of the ketone obtained from the protocol outlined in the previous paragraph $(1.80 \mathrm{~g}, 4.17 \mathrm{mmol})$ in $80 \mathrm{ml}$ toluene at $-90^{\circ} \mathrm{C}$ was added MeLi (18 ml of $1.6 \mathrm{M}$ in diethyl ether, $29 \mathrm{mmol}$ ) dropwise. After stirring the reaction mixture for $1 \mathrm{~h}$ at $-90^{\circ} \mathrm{C}$, the reaction was quenched with sat. $\mathrm{NH}_{4} \mathrm{Cl}$ (aq., $50 \mathrm{ml}$ ) and allowed to warm to RT. The aqueous phase was extracted with $\mathrm{CH}_{2} \mathrm{Cl}_{2}(3 \times 20 \mathrm{ml})$ and the combined organic extracts were dried $\left(\mathrm{Na}_{2} \mathrm{SO}_{4}\right)$ and concentrated. Flash chromatography (20:1 hexanes:ethyl acetate) provided the corresponding tertiary alcohol $(1.56 \mathrm{~g}, 84 \%)$ as a colorless oil.

To a solution of the tertiary alcohol from above $(0.80 \mathrm{~g}, 1.67 \mathrm{mmol})$ in $\mathrm{CH}_{2} \mathrm{Cl}_{2}(27 \mathrm{ml})$ was added $\mathrm{pH} 7$ buffer $(3 \mathrm{ml})$ and DDQ $(0.76 \mathrm{~g}, 3.4 \mathrm{mmol})$. The reaction mixture was stirred at RT for $2 \mathrm{~h}$ before $\mathrm{H}_{2} \mathrm{O}(20 \mathrm{ml})$ was added. The aqueous phase was extracted with ethyl acetate $(3 \times 15 \mathrm{ml})$ and the combined organic extracts were dried $\left(\mathrm{Na}_{2} \mathrm{SO}_{4}\right)$, and concentrated. Flash chromatography (2:1 hexanes:ethyl acetate) gave the corresponding diol as a colorless oil $(0.57 \mathrm{~g}, 95 \%)$.

To a solution of the diol from above $(0.11 \mathrm{~g}, 0.31 \mathrm{mmol})$ in a mixture of $\mathrm{CH}_{2} \mathrm{Cl}_{2}(6 \mathrm{ml})$ and DMSO $(3 \mathrm{ml})$ were added $\mathrm{Et}_{3} \mathrm{~N}(0.21 \mathrm{ml}, 1.65 \mathrm{mmol})$ and $\mathrm{SO}_{3} \bullet \mathrm{Py}(0.24 \mathrm{~g}, 1.65 \mathrm{mmol})$. The reaction mixture was stirred for $3 \mathrm{~h}$ at $\mathrm{RT}$ before the reaction was quenched with $\mathrm{H}_{2} \mathrm{O}(10 \mathrm{ml})$. The aqueous phase was extracted with $\mathrm{CH}_{2} \mathrm{Cl}_{2}(3 \times 5 \mathrm{ml})$ and the combined organic extracts were dried $\left(\mathrm{Na}_{2} \mathrm{SO}_{4}\right)$, and concentrated. Flash chromatography (10:1 hexanes:ethyl acetate) gave 32 as a colorless oil $(0.10 \mathrm{~g}, 91 \%) . R_{\mathrm{f}} 0.60$ (5:1 hexanes:ethyl acetate); $[\alpha]^{20}{ }_{\mathrm{D}}=-54.2^{\circ}(c=0.57, \mathrm{THF}) ;{ }^{1} \mathrm{H}$ NMR $\left(500 \mathrm{MHz}, \mathrm{CDCl}_{3}\right) \delta 5.72$ (dddd, $J=17.0, \quad 10.0, \quad 6.6, \quad 6.6 \mathrm{~Hz}, 1 \mathrm{H}), 4.98$ (dd, $J=17.0,1.3 \mathrm{~Hz}, 1 \mathrm{H}), 4.93$ $(\mathrm{d}, J=10.0 \mathrm{~Hz}, 1 \mathrm{H}), 4.56(\mathrm{~d}, J=9.7 \mathrm{~Hz}, 1 \mathrm{H}), 4.14(\mathrm{dd}, J=10.2,4.6 \mathrm{~Hz}, 1 \mathrm{H})$, $3.98(\mathrm{~s}, 1 \mathrm{H}), 3.89(\mathrm{dd}, J=10.1,10.1 \mathrm{~Hz}, 1 \mathrm{H}), 3.34(\mathrm{ddd}, J=9.8,9.8,4.6 \mathrm{~Hz}$, $1 \mathrm{H}), 3.18$ (dd, $J=9.9,2.1 \mathrm{~Hz}, 1 \mathrm{H}), 2.32(\mathrm{dd}, J=14.4,7.1 \mathrm{~Hz}, 1 \mathrm{H}), 2.13$ (ddd, $J=16.1,9.8,6.4 \mathrm{~Hz}, 1 \mathrm{H}), 1.22(\mathrm{~s}, 3 \mathrm{H}), 0.93$ (s, 9H), 0.87 (s, 9H); ${ }^{13} \mathrm{C}$ NMR $\left(125 \mathrm{MHz}, \mathrm{CDCl}_{3}\right) \delta 206.4,134.8,117.2,85.9,78.4,77.9,77.7,66.9,32.9$, 27.4, 27.1, 22.8, 20.2, 19.9; IR (neat) 3482, 2937, 2863, 1736, 1473, 1390, 1162, $1112 \mathrm{~cm}^{-1}$; ESI/MS $(\mathrm{m} / z)$ calcd for $\mathrm{C}_{18} \mathrm{H}_{32} \mathrm{O}_{5} \mathrm{SiNa} 379.2\left(\mathrm{M}+\mathrm{Na}^{+}\right)$, found 379.2 .

(4aR,6S,7R,8R,8aS)-6-(3-hydroxypropyl)-7-methylhexahydropyrano[3,2-d][1,3,2] dioxasiline-7,8-diol (34). To a solution of $32(1.56 \mathrm{~g}, 4.38 \mathrm{mmol})$ in $\mathrm{CH}_{2} \mathrm{Cl}_{2}$ $(40 \mathrm{ml})$ at $0{ }^{\circ} \mathrm{C}$ was added 2,6-lutidine $(3.10 \mathrm{ml}, 26.6 \mathrm{mmol})$ and TMSOTf $(2.38 \mathrm{ml}, 13.3 \mathrm{mmol})$. The reaction mixture was allowed to slowly warm up to $\mathrm{RT}$ over $2 \mathrm{~h}$ before it was quenched with $\mathrm{H}_{2} \mathrm{O}(20 \mathrm{ml})$. The aqueous phase was extracted with $\mathrm{CH}_{2} \mathrm{Cl}_{2}(3 \times 10 \mathrm{ml})$. The combined organic phase was dried $\left(\mathrm{Na}_{2} \mathrm{SO}_{4}\right)$ and concentrated. Flash chromatography (20:1 hexanes:ethyl acetate) gave the TMS ether as a colorless oil $(1.90 \mathrm{~g}, 100 \%)$.

To a solution of TMS ether $(1.90 \mathrm{~g}, 4.44 \mathrm{mmol})$ in THF $(40 \mathrm{ml})$ at $-78{ }^{\circ} \mathrm{C}$ was added L-Selectride $(6.7 \mathrm{ml}$ of $1.0 \mathrm{~m}$ solution in THF, $6.7 \mathrm{mmol})$. The reaction mixture was allowed to slowly warm to RT over $2 \mathrm{~h}$ before the reaction was quenched with sat. $\mathrm{NH}_{4} \mathrm{Cl}$ (aq., $20 \mathrm{ml}$ ). The aqueous phase was extracted with $\mathrm{CH}_{2} \mathrm{Cl}_{2}(3 \times 10 \mathrm{ml})$. The combined organic extracts wer dried $\left(\mathrm{Na}_{2} \mathrm{SO}_{4}\right)$ and concentrated. The resulting residue was passed through a plug of silica gel (10:1 hexanes:ethyl acetate) and concentrated giving a colorless oil that was used in the subsequente transformation without additional purification.

To a solution of the colorless oil obtained from the above procedure in THF $(15 \mathrm{ml})$ at $0{ }^{\circ} \mathrm{C}$ was added $\mathrm{BH}_{3} \bullet \mathrm{DMS}(2.0 \mathrm{M}$ solution in THF, $11 \mathrm{ml}, 22 \mathrm{mmol})$. $\mathrm{H}_{2} \mathrm{O}(1.0 \mathrm{ml}), \mathrm{NaOH}\left(25 \mathrm{ml}\right.$ of $3.0 \mathrm{M}$ aq. solution), and $\mathrm{H}_{2} \mathrm{O}_{2}$ (30 $\mathrm{ml}$ of $30 \%$ aq. solution) were added after $2 \mathrm{~h}$. The reaction mixture was warmed to RT and stirred overnight. The aqueous phase was extracted with ethyl acetate $(3 \times 20 \mathrm{ml})$. The combined organic exgtracts were dried $\left(\mathrm{Na}_{2} \mathrm{SO}_{4}\right)$ and concentrated. Flash chromatography gave 34 as a colorless oil $(1.17 \mathrm{~g}, 70 \%$ over 2 steps). $R_{\mathrm{f}} 0.35$ (1:1 hexanes:ethyl acetate); $[\alpha]^{20}{ }_{\mathrm{D}}=-39.3^{\circ}(c=0.29$, THF); ${ }^{1} \mathrm{H}$ NMR $\left(500 \mathrm{MHz}, \mathrm{CDCl}_{3}\right) \delta 4.10(\mathrm{dd}, J=9.5,4.6 \mathrm{~Hz}, 1 \mathrm{H}), 3.78$ (dd, $J=9.3,2.7 \mathrm{~Hz}, 1 \mathrm{H}), 3.73(\mathrm{dd}, J=10.2,10.2 \mathrm{~Hz}, 1 \mathrm{H}), 3.71-3.63(\mathrm{~m}, 2 \mathrm{H})$, $3.58-3.53(\mathrm{~m}, 2 \mathrm{H}), 3.43(\mathrm{~d}, J=10.2 \mathrm{~Hz}, 1 \mathrm{H}), 3.40$ (br s, $1 \mathrm{H}), 3.16$ (br s, $1 \mathrm{H})$, 3.07 (br s, $1 \mathrm{H}), 1.76(\mathrm{ddd}, J=14.2,7.1,7.1 \mathrm{~Hz}, 1 \mathrm{H}), 1.71-1.62(\mathrm{~m}, 1 \mathrm{H})$, 1.60-1.50 (m, 1H), 1.29-1.21 (m, 1H), $1.08(\mathrm{~s}, 3 \mathrm{H}), 0.98(\mathrm{~s}, 9 \mathrm{H}), 0.95(\mathrm{~s}, 9 \mathrm{H})$; ${ }^{13} \mathrm{C}$ NMR $\left(125 \mathrm{MHz}, \mathrm{CDCl}_{3}\right) \delta 78.9,75.8,74.3,71.8,70.9,67.0,62.5,30.1$, 27.6, 27.4, 24.6, 22.9, 20.4, 18.8; IR (neat) 3414, 2936, 2893, 2862, 1472, 1393, 1157, $1102 \mathrm{~cm}^{-1}$; ESI/MS $(\mathrm{m} / z)$ calcd for $\mathrm{C}_{18} \mathrm{H}_{36} \mathrm{O}_{6} \mathrm{SiNa} 399.2\left(\mathrm{M}+\mathrm{Na}^{+}\right)$, found 399.3 .
(2R,3R,4R,5S,6S)-4,5-bis(benzyloxy)-6-(3-(benzyloxy)propyl)-2-(hydroxymethyl)5-methyltetrahydro-2H-pyran-3-ol (35). To a solution of $34(1.17 \mathrm{~g}$, $3.11 \mathrm{mmol})$ in THF $(40 \mathrm{ml})$ was added $\mathrm{KH}(3.0 \mathrm{~g}$ of $30 \%$ dispersion in mineral oil, $22 \mathrm{mmol}$ ). The mixture was stirred at RT for $30 \mathrm{~min}$ and then cooled to $0{ }^{\circ} \mathrm{C}$. To the resulting mixture was added $\mathrm{BnBr}(4.22 \mathrm{ml}, 35.5 \mathrm{mmol})$ dropwise. The reaction mixture was warmed to RT and stirred overnight before the reaction was quenched with sat. $\mathrm{NH}_{4} \mathrm{Cl}$ (aq., $20 \mathrm{ml}$ ). The aqueous phase was extracted with $\mathrm{CH}_{2} \mathrm{Cl}_{2}(3 \times 20 \mathrm{ml})$. The combined organic extracts were dried $\left(\mathrm{Na}_{2} \mathrm{SO}_{4}\right)$ and concentrated to give a yellow oil which was taken to the next step without additional purification.

To the residue from above in THF $(20 \mathrm{ml})$ at $0{ }^{\circ} \mathrm{C}$ was added $\mathrm{HF} \bullet P y$ $(1.0 \mathrm{ml}, 55 \mathrm{mmol})$. The resulting reaction mixture was allowed to warm up to RT overnight. The reaction was quenched with sat. $\mathrm{NaHCO}_{3}$ (aq., $20 \mathrm{ml}$ ) and the aqueous phase was extracted with ethyl acetate $(3 \times 20 \mathrm{ml})$. The organic extracts were combined, dried $\left(\mathrm{Na}_{2} \mathrm{SO}_{4}\right)$ and concentrated. Flash chromatography (1:1 hexanes:ethyl acetate) provided diol 35 ( $0.98 \mathrm{~g}, 61 \%$ over two steps) as a colorless oil. $R_{\mathrm{f}} 0.40\left(1: 1\right.$ hexanes:ethyl acetate); $[\alpha]^{20}{ }_{\mathrm{D}}=-31.4^{\circ}(c=0.18$, THF); ${ }^{1} \mathrm{H}$ NMR $\left(500 \mathrm{MHz}, \mathrm{CDCl}_{3}\right) \delta 7.50-7.35(\mathrm{~m}, 15 \mathrm{H}), 5.02(\mathrm{~d}, J=11.2 \mathrm{~Hz}$, $1 \mathrm{H}), 4.80(\mathrm{~d}, J=11.2 \mathrm{~Hz}, 1 \mathrm{H}), 4.72(\mathrm{~d}, J=11.5 \mathrm{~Hz}, 1 \mathrm{H}), 4.66(\mathrm{~d}, J=11.5 \mathrm{~Hz}$, $1 \mathrm{H}), 4.62(\mathrm{~s}, 2 \mathrm{H}), 4.08(\mathrm{~d}, J=3.9 \mathrm{~Hz}, 1 \mathrm{H}), 3.98(\mathrm{~d}, J=10.2 \mathrm{~Hz}, 1 \mathrm{H}), 3.95$ (partially obscured br d, $J=14.6 \mathrm{~Hz}, 1 \mathrm{H}$ ), 3.88-3.76 (m, 2H), 3.70 (ddd, $J=10.0,3.4,3.4 \mathrm{~Hz}, 1 \mathrm{H}), 3.66-3.62(\mathrm{~m}, 2 \mathrm{H}), 3.14-3.04(\mathrm{~m}, 2 \mathrm{H}), 2.10-1.98$ $(\mathrm{m}, 2 \mathrm{H}), 1.90-1.78(\mathrm{~m}, 1 \mathrm{H}), 1.60-1.48(\mathrm{~m}, 1 \mathrm{H}), 1.38(\mathrm{~s}, 3 \mathrm{H}) ;{ }^{13} \mathrm{C}$ NMR $\left(125 \mathrm{MHz}, \mathrm{CDCl}_{3}\right) \delta 139.2,139.1,139.0,128.8,128.7,128.0,127.9,127.8$, $127.7,127.3,81.2,78.1,76.9,76.8,75.4,73.1,70.8,68.0,63.9,63.2,27.3,25.3$, 16.6; IR (neat) 3421, 2930, 2874, 1496, 1454, 1380, 1210, $1106 \mathrm{~cm}^{-1}$; ESI/MS $(\mathrm{m} / \mathrm{z})$ calcd for $\mathrm{C}_{31} \mathrm{H}_{38} \mathrm{O}_{6} \mathrm{Na} 529.3\left(\mathrm{M}+\mathrm{Na}^{+}\right)$, found 529.3.

\section{(2R,3R,4R,5S,6S)-4,5-bis(benzyloxy)-6-(3-(benzyloxy)propyl)-5-methyl-2-}

(prop-2-yn-1-yl)tetrahydro-2H-pyran-3-ol (37). To a solution of $35(0.57 \mathrm{~g}$, $1.13 \mathrm{mmol})$ and 2,6-lutidine $(0.495 \mathrm{ml}, 4.25 \mathrm{mmol})$ in $\mathrm{CH}_{2} \mathrm{Cl}_{2}(20 \mathrm{ml})$ at $-78^{\circ} \mathrm{C}$ was added trifluoromethanesulfonic anhydride $(0.192 \mathrm{ml}, 0.114 \mathrm{mmol})$. After stirring for $30 \mathrm{~min}$ TESOTf $(0.288 \mathrm{ml}, 0.127 \mathrm{mmol})$ was added to the reaction mixture. After warming to $\mathrm{RT}$ the reaction was quenched with sat. $\mathrm{NaHCO}_{3}$ (aq., $10 \mathrm{ml}$ ). The aqueous phase was extracted with $\mathrm{CH}_{2} \mathrm{Cl}_{2}$ $(3 \times 10 \mathrm{ml})$. The combined organic extracts were dried $\left(\mathrm{Na}_{2} \mathrm{SO}_{4}\right)$ and concentrated to give a yellow oil. The oil was passed through a short plug of silica gel (10:1 hexanes:ethyl acetate) and concentrated to give triflate 36 .

To a solution of trimethylsilylacetylene $(0.690 \mathrm{ml}, 4.88 \mathrm{mmol})$ in THF $(20 \mathrm{ml})$ at $0{ }^{\circ} \mathrm{C}$ was added $n \mathrm{BuLi}(1.94 \mathrm{ml}$ of $2.5 \mathrm{M}$ solution in hexanes, $4.85 \mathrm{mmol}$ ). The reaction mixture was stirred for $30 \mathrm{~min}$ and then cooled to $-78^{\circ} \mathrm{C}$. To the resulting solution of acetylide was added a solution of $\mathbf{3 6}$ and HMPA $(0.900 \mathrm{ml}, 5.17 \mathrm{mmol})$ in THF $(10 \mathrm{ml})$ via cannula. The reaction mixture was warmed to RT before the reaction was quenched with sat. $\mathrm{NH}_{4} \mathrm{Cl}$ (aq., $10 \mathrm{ml})$. The aqueous phase was extracted with $\mathrm{CH}_{2} \mathrm{Cl}_{2}(3 \times 10 \mathrm{ml})$. The combined organic extracts were dried $\left(\mathrm{Na}_{2} \mathrm{SO}_{4}\right)$ and concentrated to give a dark-brown oil, which was taken up in THF $(15 \mathrm{ml})$. To the solution was added TBAF $(4.90 \mathrm{ml}$ of $1.0 \mathrm{M}$ solution in THF, $4.90 \mathrm{mmol}$ ) and the mixture was stirred at RT overnight before it was quenched with sat. $\mathrm{NH}_{4} \mathrm{Cl}$ (aq., $10 \mathrm{ml}$ ). The aqueous phase was extracted with $\mathrm{CH}_{2} \mathrm{Cl}_{2}(3 \times 10 \mathrm{ml})$. The combined organic phase was dried $\left(\mathrm{Na}_{2} \mathrm{SO}_{4}\right)$ and concentrated. Flash chromatography (4:1 hexanes:ethyl acetate) gave 37 as a colorless oil $(0.34 \mathrm{~g}, 59 \%$ over three steps). $R_{\mathrm{f}} 0.20$ (4:1 hexanes:ethyl acetate); $[\alpha]^{20}{ }_{\mathrm{D}}=-21.6^{\circ} \quad(c=0.45$, THF); ${ }^{1} \mathrm{H}$ NMR $\left(500 \mathrm{MHz}, \mathrm{CDCl}_{3}\right) \delta 7.42-7.27(\mathrm{~m}, 15 \mathrm{H}), 5.01(\mathrm{~d}, J=11.0 \mathrm{~Hz}, 1 \mathrm{H})$, $4.72(\mathrm{~d}, J=11.5 \mathrm{~Hz}, 1 \mathrm{H}), 4.65(\mathrm{~d}, J=11.5 \mathrm{~Hz}, 1 \mathrm{H}), 4.56(\mathrm{~s}, 2 \mathrm{H}), 4.54$ $(\mathrm{d}, J=11.5 \mathrm{~Hz}, 1 \mathrm{H}), 4.02(\mathrm{~d}, J=1.7 \mathrm{~Hz}, 1 \mathrm{H}), 3.85(\mathrm{~d}, J=9.8 \mathrm{~Hz}, 1 \mathrm{H})$, $3.64-3.58(\mathrm{~m}, 4 \mathrm{H}), 2.67$ (ddd, $J=17.1,2.4,2.4 \mathrm{~Hz}, 1 \mathrm{H}), 2.47$ (ddd, $J=17.1$, $5.9,2.7 \mathrm{~Hz}, 1 \mathrm{H}), 2.30-2.23(\mathrm{~m}, 1 \mathrm{H}), 2.01(\mathrm{t}, J=2.7 \mathrm{~Hz}, 1 \mathrm{H}), 2.00-1.90$ $(\mathrm{m}, 2 \mathrm{H}), 1.81-1.70(\mathrm{~m}, 1 \mathrm{H}), 1.50-1.40(\mathrm{~m}, 1 \mathrm{H}), 1.34(\mathrm{~s}, 3 \mathrm{H}) ;{ }^{13} \mathrm{C}$ NMR $\left(125 \mathrm{MHz}, \mathrm{CDCl}_{3}\right) \delta 139.1,138.9,138.7,128.8,128.6,128.5,128.2,128.0$, $127.8,127.6,127.5,127.1,81.3,81.2,78.3,77.0,75.5,75.2,73.0,70.8,69.9$, 64.0, 27.1, 25.1, 22.4, 16.2; IR (neat) 3420, 2928, 2873, 2119, 1606, 1496, 1454, $1378,1209,1104 \mathrm{~cm}^{-1}$; ESI/MS $(\mathrm{m} / \mathrm{z})$ calcd for $\mathrm{C}_{33} \mathrm{H}_{38} \mathrm{O}_{5} \mathrm{~K} 553.3\left(\mathrm{M}+\mathrm{K}^{+}\right)$, found 553.2.

$(((2 R, 3 R, 4 R, 5 S, 6 S)-2$-allyl-4,5-bis(benzyloxy)-6-(3-(benzyloxy)propyl)-5-methyltetrahydro-2H-pyran-3-yl)oxy)triethylsilane (38). To a solution of 37 (600 mg, 
$1.17 \mathrm{mmol})$ in $\mathrm{CH}_{2} \mathrm{Cl}_{2}(20 \mathrm{ml})$ at $0{ }^{\circ} \mathrm{C}$ was added 2,6-lutidine $(0.272 \mathrm{ml}$, $2.34 \mathrm{mmol})$ and TESOTf $(0.343 \mathrm{ml}, 1.52 \mathrm{mmol})$. The reaction mixture was allowed to slowly warm to RT over $3 \mathrm{~h}$ before the reaction was quenched with sat. $\mathrm{NaHCO}_{3}$ (aq., $10 \mathrm{ml}$ ). The aqueous phase was extracted with $\mathrm{CH}_{2} \mathrm{Cl}_{2}$ $(3 \times 10 \mathrm{ml})$ and the combined organic extracts were dried $\left(\mathrm{Na}_{2} \mathrm{SO}_{4}\right)$ and concentrated. Flash chromatography (5:1 hexanes:ethyl acetate) provided the corresponding TES ether $(732 \mathrm{mg}, 100 \%)$ as a colorless oil.

To a solution of the TES ether obtained from above in ethyl acetate $(30 \mathrm{ml})$ was added quinoline $(70 \mu \mathrm{l}, 0.59 \mathrm{mmol})$ and Lindlar's Pd catalyst $(50 \mathrm{mg})$. The reaction mixture was stirred $\mathrm{H}_{2}(1 \mathrm{~atm})$ for $2 \mathrm{~h}$ before being passed through a Celite plug using ethyl acetate. The filtrate was concentrated and flash chromatography (10:1 hexanes:ethyl acetate) gave $\mathbf{3 8}$ as a colorless oil (638 mg, 87\%). $R_{\mathrm{f}} 0.50$ (15:2 hexanes:ethyl acetate); $[\alpha]^{20}{ }_{\mathrm{D}}=+1.2^{\circ}(c=0.45$, THF); ${ }^{1} \mathrm{H} \quad \mathrm{NMR}\left(500 \mathrm{MHz}, \mathrm{C}_{6} \mathrm{D}_{6}\right) \quad \delta \quad 7.44 \quad(\mathrm{~d}, J=7.3 \mathrm{~Hz}, \quad 2 \mathrm{H}), \quad 7.33$ (d, $J=7.3 \mathrm{~Hz}, 2 \mathrm{H}), 7.28$ (d, $J=7.3 \mathrm{~Hz}, 2 \mathrm{H}), 7.20-7.04$ (m, 9H), 6.08 (dddd, $J=17.1, \quad 11.2,6.8, \quad 6.8 \mathrm{~Hz}, 1 \mathrm{H}), 5.19(\mathrm{dd}, J=17.1,1.9 \mathrm{~Hz}, 1 \mathrm{H}), 5.11$ $(\mathrm{d}, J=6.8 \mathrm{~Hz}, 1 \mathrm{H}), 5.08(\mathrm{dd}, J=8.8,1.9 \mathrm{~Hz}, 1 \mathrm{H}), 4.68(\mathrm{~d}, J=11.2 \mathrm{~Hz}, 1 \mathrm{H})$, $4.42(\mathrm{~d}, J=10.7 \mathrm{~Hz}, 1 \mathrm{H}), 4.38(\mathrm{~d}, J=11.3 \mathrm{~Hz}, 1 \mathrm{H}), 4.29(\mathrm{~s}, 2 \mathrm{H}), 4.05-4.00$ $(\mathrm{m}, 2 \mathrm{H}), 3.82(\mathrm{~d}, J=2.4 \mathrm{~Hz}, 1 \mathrm{H}), 3.61(\mathrm{dd}, J=9.3,2.0 \mathrm{~Hz}, 1 \mathrm{H}), 3.39-3.31$ $(\mathrm{m}, 2 \mathrm{H}), 2.68(\mathrm{dd}, J=13.2,6.8 \mathrm{~Hz}, 1 \mathrm{H}), 2.24(\mathrm{ddd}, J=15.6,7.8,7.8 \mathrm{~Hz}, 1 \mathrm{H})$, 2.06-1.95 (m, 2H), 1.70-1.60 (m, 1H), 1.56-1.48 (m, 1H), $1.19(\mathrm{~s}, 3 \mathrm{H})$, $1.00(\mathrm{t}, J=7.8 \mathrm{~Hz}, 9 \mathrm{H}), 0.64(\mathrm{q}, J=7.8 \mathrm{~Hz}, 6 \mathrm{H}), 0.30(\mathrm{~s}, 3 \mathrm{H}) ;{ }^{13} \mathrm{C} \mathrm{NMR}$ $\left(125 \mathrm{MHz}, \mathrm{C}_{6} \mathrm{D}_{6}\right) \delta 140.3,140.1,139.9,136.2,128.9,128.8,128.8,128.0,127.9$, $127.8,127.8,127.7,117.0,83.0,78.0,77.7,76.4,76.0,74.7,73.1,71.1,63.9$, $37.3,27.7,26.0,17.7,7.7,5.9$; IR (neat) 2955, 2876, 1454, 1379, 1239, 1107, $1027 \mathrm{~cm}^{-1}$; ESI/MS $(\mathrm{m} / z)$ calcd for $\mathrm{C}_{39} \mathrm{H}_{54} \mathrm{O}_{5} \mathrm{SiNa} 653.4 \quad\left(\mathrm{M}+\mathrm{Na}^{+}\right)$, found 653.4 .

\section{2-((2R,3R,4R,5S,6S)-4,5-bis(benzyloxy)-6-(3-(benzyloxy)propyl)-5-methyl-3-} ((triethylsilyl)oxy)tetrahydro-2H-pyran-2-yl)acetic acid (38). $\mathrm{O}_{3}$ was bubbled through a solution of $38(638 \mathrm{mg}, 1.01 \mathrm{mmol})$ in $\mathrm{CH}_{2} \mathrm{Cl}_{2}(60 \mathrm{ml})$ at $-78{ }^{\circ} \mathrm{C}$ until the reaction mixture remained light blue in color. The excess $\mathrm{O}_{3}$ was purged from the reaction mixture by bubbling $\mathrm{N}_{2}$ through it until the light blue color completely dissipated (ca. $10 \mathrm{~min}$ ). Triphenylphosphine (1.33 g, $5.08 \mathrm{mmol}$ ) was then added and the mixture was allowed to slowly warm to RT. After $12 \mathrm{~h}$, the solution was concentrated under reduced pressure. Flash chromatography (50:1 to 5:1 hexanes:ethyl acetate) provided the aldehyde (600 mg, 94\%) as a colorless oil.

To a solution of the aldehyde from the ozonolysis $(600 . \mathrm{mg}, 0.949 \mathrm{mmol})$ in THF $(10 \mathrm{ml})$ at $0{ }^{\circ} \mathrm{C}$ was added $t \mathrm{BuOH}(10 \mathrm{ml}), \mathrm{H}_{2} \mathrm{O}(10 \mathrm{ml}), 2$-Me-2-butene (2.0 ml), $\mathrm{NaH}_{2} \mathrm{PO}_{4}(570 \mathrm{mg}, 0.475 \mathrm{mmol})$ and $\mathrm{NaClO}_{2}(430 \mathrm{mg}, 0.48 \mathrm{mmol})$. The reaction was quenched after $30 \mathrm{~min}$ by adding $\mathrm{H}_{2} \mathrm{O}(30 \mathrm{ml})$. The resulting mixture was extracted with ethyl acetate $(3 \times 15 \mathrm{ml})$ and the organic extracts were combined, dried $\left(\mathrm{Na}_{2} \mathrm{SO}_{4}\right)$ and concentrated. Flash chromatography (1:1 hexanes:ethyl acetate to ethyl acetate) provided acid $39(615 \mathrm{mg}, 100 \%)$ as a colorless oil. $R_{\mathrm{f}} 0.50$ (2:1 hexanes:ethyl acetate); $[\alpha]^{20}{ }_{\mathrm{D}}=-9.4^{\circ}(c=0.27$, THF); ${ }^{1} \mathrm{H}$ NMR (500 MHz, $\left.\mathrm{C}_{6} \mathrm{D}_{6}\right) \delta 11.7$ (br s, $\left.1 \mathrm{H}\right), 7.44(\mathrm{~d}, J=7.8 \mathrm{~Hz}, 2 \mathrm{H})$, $7.32(\mathrm{~d}, J=7.8 \mathrm{~Hz}, 2 \mathrm{H}), 7.29(\mathrm{~d}, J=7.4 \mathrm{~Hz}, 2 \mathrm{H}), 7.19-7.03(\mathrm{~m}, 9 \mathrm{H}), 5.09$ $(\mathrm{d}, J=11.2 \mathrm{~Hz}, 1 \mathrm{H}), 4.65(\mathrm{~d}, J=11.2 \mathrm{~Hz}, 1 \mathrm{H}), 4.52(\mathrm{ddd}, J=8.8,8.8,3.4 \mathrm{~Hz}$, $1 \mathrm{H}), 4.41(\mathrm{~d}, J=11.2 \mathrm{~Hz}, 1 \mathrm{H}), 4.37(\mathrm{~d}, J=11.2 \mathrm{~Hz}, 1 \mathrm{H}), 4.31(\mathrm{~s}, 2 \mathrm{H}), 4.11$ $(\mathrm{d}, J=10.7 \mathrm{~Hz}, 1 \mathrm{H}), 3.82(\mathrm{~d}, J=2.0 \mathrm{~Hz}, 1 \mathrm{H}), 3.73(\mathrm{dd}, J=9.2,2.0 \mathrm{~Hz}, 1 \mathrm{H})$, $3.40-3.32(\mathrm{~m}, 2 \mathrm{H}), 2.86(\mathrm{dd}, J=15.4,3.2 \mathrm{~Hz}, 1 \mathrm{H}), 2.54(\mathrm{dd}, J=15.4,9.0 \mathrm{~Hz}$, $1 \mathrm{H}), 2.02-1.92(\mathrm{~m}, 2 \mathrm{H}), 1.72-1.62(\mathrm{~m}, 1 \mathrm{H}), 1.50(\mathrm{ddd}, J=9.3,9.3,9.3 \mathrm{~Hz}, 1 \mathrm{H})$, $1.16(\mathrm{~s}, \quad 3 \mathrm{H}), 0.96(\mathrm{t}, J=7.8 \mathrm{~Hz}, 9 \mathrm{H}), 0.58 \quad(\mathrm{q}, J=7.8 \mathrm{~Hz}, 6 \mathrm{H}) ;{ }^{13} \mathrm{C}$ NMR $\left(125 \mathrm{MHz}, \mathrm{C}_{6} \mathrm{D}_{6}\right) \delta 178.3,140.1,140.0,139.9,128.9,128.8,128.8$, $128.1,127.9,127.8,127.8,127.7,82.8,78.0,77.9,76.0,73.8,73.8,73.0,70.8$, 63.9, 38.2, 27.4, 25.7, 17.6, 7.6, 5.7; IR (neat) 3330, 2956, 2877, 1713, 1454, $1241,1111 \mathrm{~cm}^{-1}$; ESI/MS $(\mathrm{m} / \mathrm{z})$ calcd for $\mathrm{C}_{38} \mathrm{H}_{52} \mathrm{O}_{7} \mathrm{SiNa} 671.4\left(\mathrm{M}+\mathrm{Na}^{+}\right)$, found 671.3 .

$(2 R, 4 a R, 6 S, 7 R, 8 a S)-2-(4-m e t h o x y p h e n y l)-4 a, 6$-dimethyl-6-(pent-4-en-1-yl) hexahydropyrano [3,2-d] [1,3]dioxin-7-yl 2-((2R,3R,4R,5S,6S)-4,5-bis(benzyloxy)6-(3-(benzyloxy)propyl)-5-methyl-3-((triethylsilyl)oxy)tetrahydro-2H-pyran-2-yl) acetate (40). To a solution of acid $39(53.4 \mathrm{mg}, 82.2 \mu \mathrm{mol})$ in THF $(8 \mathrm{ml})$ was added triethylamine $(0.100 \mathrm{ml}, 0.719 \mathrm{mmol})$ and 2,4,6-trichlorobenzoyl chloride $(77.0 \mu \mathrm{l}, 0.493 \mathrm{mmol})$. The reaction mixture was heated at $40^{\circ} \mathrm{C}$ for $2 \mathrm{~h}$ before the solvent was removed in vacuo. To the resulting residue was transfered a solution of alcohol $30(24.8 \mathrm{mg}, 68.5 \mu \mathrm{mol})$ in toluene $(8 \mathrm{ml})$ followed by DMAP $(0.100 \mathrm{~g}, 0.820 \mathrm{mmol})$. The resulting reaction mixture was heated at $40{ }^{\circ} \mathrm{C}$ for $2 \mathrm{~h}$ and then the reaction was quenched with sat. $\mathrm{NaHCO}_{3}$ (aq., $10 \mathrm{ml})$. The aqueous phase was extracted with $\mathrm{CH}_{2} \mathrm{Cl}_{2}(3 \times 10 \mathrm{ml})$, the organic extracts were combined, dried $\left(\mathrm{Na}_{2} \mathrm{SO}_{4}\right)$ and concentrated. Flash chromatography (10:1 hexanes:ethyl acetate) provided ester $40(57.4 \mathrm{mg}, 84 \%)$ as a colorless oil. $R_{\mathrm{f}} 0.70$ (4:1 hexanes:ethyl acetate); $[\alpha]^{20} \mathrm{D}=-26.2^{\circ}(c=0.29$, THF); ${ }^{1} \mathrm{H} \quad \mathrm{NMR}\left(500 \mathrm{MHz}, \mathrm{C}_{6} \mathrm{D}_{6}\right) \quad \delta \quad 7.55(\mathrm{~d}, J=8.8 \mathrm{~Hz}, \quad 2 \mathrm{H}), \quad 7.44$ (d, $J=7.3 \mathrm{~Hz}, 2 \mathrm{H}), 7.35(\mathrm{~d}, J=7.3 \mathrm{~Hz}, 2 \mathrm{H}), 7.29(\mathrm{~d}, J=7.8 \mathrm{~Hz}, 2 \mathrm{H})$, 7.20-7.05 (m, 9H), 6.82 (d, $J=8.3 \mathrm{~Hz}, 2 \mathrm{H}), 5.84$ (dddd, $J=17.1,10.3,6.8$, $6.8 \mathrm{~Hz}, 1 \mathrm{H}), 5.32(\mathrm{~s}, 1 \mathrm{H}), 5.22(\mathrm{dd}, J=11.2,4.9 \mathrm{~Hz}, 1 \mathrm{H}), 5.11(\mathrm{~d}, J=11.2 \mathrm{~Hz}$, $1 \mathrm{H}), 5.08$ (dd, $J=15.6,1.5 \mathrm{~Hz}, 1 \mathrm{H}), 5.02(\mathrm{dd}, J=10.3,1.0 \mathrm{~Hz}, 1 \mathrm{H}), 4.68$ (d, $J=11.2 \mathrm{~Hz}, 1 \mathrm{H}), 4.49$ (ddd, $J=10.3,7.3,3.4 \mathrm{~Hz}, 1 \mathrm{H}), 4.43(\mathrm{~d}, J=11.2 \mathrm{~Hz}$, $1 \mathrm{H}), 4.38(\mathrm{~d}, J=11.2 \mathrm{~Hz}, 1 \mathrm{H}), 4.33(\mathrm{~s}, 2 \mathrm{H}), 4.13(\mathrm{~d}, J=9.8 \mathrm{~Hz}, 1 \mathrm{H}), 3.99$ (dd, $J=9.8,2.0 \mathrm{~Hz}, 1 \mathrm{H}), 3.88(\mathrm{~d}, J=2.0 \mathrm{~Hz}, 1 \mathrm{H}), 3.80(\mathrm{~d}, J=9.8 \mathrm{~Hz}, 1 \mathrm{H}), 3.42-3.34$ $(\mathrm{m}, 3 \mathrm{H}), 3.27(\mathrm{~s}, 3 \mathrm{H}), 3.28-3.24$ (partially obscured $\mathrm{m}, 1 \mathrm{H}), 2.86$ (dd, $J=14.7$, $3.4 \mathrm{~Hz}, 1 \mathrm{H}), 2.62(\mathrm{dd}, J=14.7,7.3 \mathrm{~Hz}, 1 \mathrm{H}), 2.29$ (ddd, $J=11.2,4.4,3.9 \mathrm{~Hz}$, $1 \mathrm{H}), 2.08-1.96(\mathrm{~m}, 5 \mathrm{H}), 1.80-1.55(\mathrm{~m}, 6 \mathrm{H}), 1.49(\mathrm{~s}, 3 \mathrm{H}), 1.30(\mathrm{~s}, 3 \mathrm{H}), 1.22$ $(\mathrm{s}, 3 \mathrm{H}), 1.00(\mathrm{t}, J=8.3 \mathrm{~Hz}, 9 \mathrm{H}), 0.67(\mathrm{q}, J=7.8 \mathrm{~Hz}, 3 \mathrm{H}), 0.66(\mathrm{q}, J=8.3 \mathrm{~Hz}$, $3 \mathrm{H}) ;{ }^{13} \mathrm{C}$ NMR (125 MHz, $\left.\mathrm{C}_{6} \mathrm{D}_{6}\right) \delta 170.7,160.8,140.2,140.0,139.8,139.5$, $131.6,128.9,128.8,128.8,128.5,128.0,127.9,127.9,127.9,127.8,127.7,115.1$, $114.1,103.1,82.6,80.5,78.1,78.0,77.3,77.1,76.0,74.3,74.0,73.2,73.0,71.0$, $69.5,63.9,55.1,42.4,37.8,34.9,30.5,28.2,27.8,26.1,23.6,23.0,19.5,17.6,7.6$, 5.8; IR (neat) 2954, 2876, 1741, 1496, 1379, 1249, $1106 \mathrm{~cm}^{-1}$; ESI/MS $(\mathrm{m} / \mathrm{z})$ calcd for $\mathrm{C}_{59} \mathrm{H}_{80} \mathrm{O}_{11} \mathrm{SiNa}$ 1015.6 $\left(\mathrm{M}+\mathrm{Na}^{+}\right)$, found 1015.5.

$(2 R, 3 R, 4 R, 5 S, 6 S)-4,5-b i s($ benzyloxy)-6-(3-(benzyloxy)propyl)-2-(((2R,4aR,5aS, 11aR,12aS,Z)-2-(4-methoxyphenyl)-4a,5a-dimethyl-4a,5a,6,7,8,11a,12,12aoctahydro-4H-[1,3]dioxino $\left[4,55^{\prime}: 5,6\right]$ pyrano[3,2-b]oxocin-10-yl)methyl)-5methyltetrahydro-2H-pyran-3-ol (41). To a solution of $\mathrm{TiCl}_{4}(0.230 \mathrm{ml}$, $2.09 \mathrm{mmol})$ in $\mathrm{CH}_{2} \mathrm{Cl}_{2}(13 \mathrm{ml})$ at $0{ }^{\circ} \mathrm{C}$ was added THF $(1.10 \mathrm{ml}, 12.5 \mathrm{mmol})$ dropwise. TMEDA $(1.90 \mathrm{ml}, 12.5 \mathrm{mmol})$ was added dropwise to the resulting yellow solution. The solution turned red-brown and the ice bath was removed. Activated $\mathrm{Zn}$ dust (304 mg, $4.68 \mathrm{mmol})$ and $\mathrm{PbCl}_{2}(69.0 \mathrm{mg}, 0.248 \mathrm{mmol})$ were added after the red-brown reaction mixture had stirred for $15 \mathrm{~min}$. The resulting slurry went through a series of color changes from brown to green to purple and finally to blue-green over the course of 3-5 min. A solution of ester $40(32.3 \mathrm{mg}, 32.5 \mu \mathrm{mol})$ and $\mathrm{CH}_{3} \mathrm{CHBr}_{2}(0.190 \mathrm{ml}, 2.12 \mathrm{mmol})$ in $\mathrm{CH}_{2} \mathrm{Cl}_{2}$ $(3 \mathrm{ml})$ was transferred to the reaction mixture via cannula. The reaction mixture was then heated to reflux for $2 \mathrm{~h}$ before it was cooled to $0{ }^{\circ} \mathrm{C}$ and the reaction was quenched with sat. $\mathrm{K}_{2} \mathrm{CO}_{3}$ (aq., $1.0 \mathrm{ml}$ ). After stirring for $30 \mathrm{~min}$ at $0{ }^{\circ} \mathrm{C}$, the resulting mixture was filtered. The filtrate was concentrated and flash chromatography (10:1 hexanes:ethyl acetate) gave $\mathbf{4 1}$ as a colorless oil (12.5 mg, 40\%). $R_{\mathrm{f}} 0.55$ (5:1 hexanes:ethyl acetate); $[\alpha]^{20}{ }_{\mathrm{D}}=-14.1^{\circ}(c=0.28$, THF); ${ }^{1} \mathrm{H} \quad \mathrm{NMR}\left(500 \mathrm{MHz}, \mathrm{C}_{6} \mathrm{D}_{6}\right) \quad \delta \quad 7.61 \quad(\mathrm{~d}, J=8.8 \mathrm{~Hz}, \quad 2 \mathrm{H}), \quad 7.50$ (d, $J=7.3 \mathrm{~Hz}, 2 \mathrm{H}), 7.38(\mathrm{~d}, J=7.3 \mathrm{~Hz}, 2 \mathrm{H}), 7.30(\mathrm{~d}, J=7.3 \mathrm{~Hz}, 2 \mathrm{H})$, 7.21-7.06 (m, 9H), $6.83 \quad(\mathrm{~d}, \quad J=8.3 \mathrm{~Hz}, \quad 2 \mathrm{H}), \quad 5.48 \quad(\mathrm{~s}, \quad 1 \mathrm{H}), \quad 5.16$ $(\mathrm{d}, J=11.2 \mathrm{~Hz}, 1 \mathrm{H}), 4.90(\mathrm{dd}, J=8.3,2.9 \mathrm{~Hz}, 1 \mathrm{H}), 4.71(\mathrm{~d}, J=11.2 \mathrm{~Hz}, 1 \mathrm{H})$, $4.47(\mathrm{~d}, J=11.2 \mathrm{~Hz}, 1 \mathrm{H}), 4.41(\mathrm{~d}, J=11.2 \mathrm{~Hz}, 1 \mathrm{H}), 4.31(\mathrm{~s}, 2 \mathrm{H}), 4.14$ $(\mathrm{d}, J=9.8 \mathrm{~Hz}, 1 \mathrm{H}), 3.98(\mathrm{dd}, J=9.8,4.9 \mathrm{~Hz}, 1 \mathrm{H}), 3.89(\mathrm{~d}, J=6.8 \mathrm{~Hz}, 1 \mathrm{H})$, $3.88(\mathrm{~s}, 1 \mathrm{H}), 3.69$ (dd, $J=9.8,2.2 \mathrm{~Hz}, 1 \mathrm{H}), 3.48(\mathrm{~d}, J=9.8 \mathrm{~Hz}, 1 \mathrm{H}), 3.44-3.38$ $(\mathrm{m}, \quad 2 \mathrm{H}), \quad 3.27(\mathrm{~s}, 3 \mathrm{H}), \quad 3.28-3.22$ (partially obscured $\mathrm{m}, 1 \mathrm{H}$ ), 2.91 (d, $J=14.6 \mathrm{~Hz}, 1 \mathrm{H}), 2.30-1.97(\mathrm{~m}, 8 \mathrm{H}), 1.84-1.70(\mathrm{~m}, 2 \mathrm{H}), 1.69-1.56$ $(\mathrm{m}, 3 \mathrm{H}), 1.55(\mathrm{~s}, 3 \mathrm{H}), 1.36(\mathrm{~s}, 3 \mathrm{H}), 1.24(\mathrm{~s}, 3 \mathrm{H}), 1.01(\mathrm{t}, J=7.8 \mathrm{~Hz}, 9 \mathrm{H})$, $0.64(\mathrm{q}, J=7.8 \mathrm{~Hz}, 6 \mathrm{H}) ;{ }^{13} \mathrm{C}$ NMR $\left(100 \mathrm{MHz}, \mathrm{C}_{6} \mathrm{D}_{6}\right) \delta 160.8,155.7,140.3$, $140.1,140.0,131.8,128.9,128.8,128.5,128.0,127.9,127.9,127.8,127.7,114.1$, 108.1, 103.2, 84.0, 83.1, 81.3, 78.5, 78.0, 77.9, 77.8, 76.1, 74.9, 74.5, 73.1, 71.1, $69.4,63.9,55.1,46.3,40.2,33.6,31.0,30.5,28.5,27.8,25.9,28.5,27.8,25.9$, 22.4, 22.1, 19.8, 17.8, 7.6, 5.8; IR (neat) 2928, 2876, 1615, 1496, 1378, 1250, $1108 \mathrm{~cm}^{-1}$; ESI/MS $(\mathrm{m} / \mathrm{z})$ calcd for $\mathrm{C}_{58} \mathrm{H}_{78} \mathrm{O}_{10} \mathrm{SiK} 1001.6\left(\mathrm{M}+\mathrm{K}^{+}\right)$, found 1001.5.

$(2 R, 4 a R, 5 a S, 10 S, 11 a R, 12 a S)-10-(((2 R, 3 R, 4 R, 5 S, 6 S)-4,5-b i s($ benzyloxy $)-6-(3-$ (benzyloxy)propyl)-3-hydroxy-5-methyltetrahydro-2H-pyran-2-yl)methyl)-2-(4methoxyphenyl)-4a,5a-dimethyloctahydro-4H-[1,3]dioxino $[4,5: 5,6]$ pyrano [3,2-b] oxocin-9(10H)-one (44). To a solution of $41(19.7 \mathrm{mg}, 20.4 \mu \mathrm{mol})$ in $\mathrm{CH}_{2} \mathrm{Cl}_{2}$ $(5 \mathrm{ml})$ at $-78^{\circ} \mathrm{C}$ was added a solution of dimethyl dioxirane $(0.42 \mathrm{ml}$ of 0.10 
M solution in $\mathrm{CH}_{2} \mathrm{Cl}_{2}, 0.042 \mathrm{mmol}$ ) dropwise. The reaction mixture was warmed to $0{ }^{\circ} \mathrm{C}$ and the solvent was removed in vacuo. The residue was dissolved in $\mathrm{CH}_{2} \mathrm{Cl}_{2}(5 \mathrm{ml})$ and the reaction mixture was cooled to $-78{ }^{\circ} \mathrm{C}$ and a solution of $i-\mathrm{Bu}_{2} \mathrm{AlH}(0.21 \mathrm{ml}$ of $1.0 \mathrm{M}$ solution in THF, $0.21 \mathrm{mmol})$ was added. After stirring for $2 \mathrm{~h}$ at $-78^{\circ} \mathrm{C}$, the reaction was quenched with sat. $\mathrm{NH}_{4} \mathrm{Cl}$ (aq., $3 \mathrm{ml}$ ) and allowed to warm to RT. A solution of saturated potassium sodium tartrate $(10 \mathrm{ml})$ was added and the mixture was stirred violently for $30 \mathrm{~min}$. The aqueous phase was extracted with $\mathrm{CH}_{2} \mathrm{Cl}_{2}(3 \times 10 \mathrm{ml})$ and the combined organic extracts were dried $\left(\mathrm{Na}_{2} \mathrm{SO}_{4}\right)$ and concentrated to give alcohol 43.

To a solution of the alcohol obtained from above in $\mathrm{CH}_{2} \mathrm{Cl}_{2}(5 \mathrm{ml})$ was added activated $4 \AA \mathrm{MS}(20 \mathrm{mg}), \mathrm{NMO}(25.0 \mathrm{mg}, 0.213 \mathrm{mmol})$ and TPAP $(1 \mathrm{mg}, 0.003 \mathrm{mmol})$. The reaction mixture was stirred at RT for $2 \mathrm{~h}$ before being concentrated. Flash chromatography (5:1 hexanes:ethyl acetate) gave ketone 44 as a colorless oil ( $12.2 \mathrm{mg}, 61 \%$ two steps). $R_{\mathrm{f}} 0.55$ (3:1 hexanes:ethyl acetate); $[\alpha]^{20}{ }_{\mathrm{D}}=-20.2^{\circ}(c=0.18, \mathrm{THF}) ;{ }^{1} \mathrm{H}$ NMR $\left(500 \mathrm{MHz}, \mathrm{C}_{6} \mathrm{D}_{6}\right) \delta 7.58$ $(\mathrm{d}, J=8.8 \mathrm{~Hz}, 2 \mathrm{H}), 7.46(\mathrm{~d}, J=7.3 \mathrm{~Hz}, 2 \mathrm{H}), 7.36(\mathrm{~d}, J=7.8 \mathrm{~Hz}, 2 \mathrm{H}), 7.30$ (d, $J=7.8 \mathrm{~Hz}, 2 \mathrm{H}), 7.21-7.06(\mathrm{~m}, 9 \mathrm{H}), 6.83(\mathrm{~d}, J=8.3 \mathrm{~Hz}, 2 \mathrm{H}), 5.36(\mathrm{~s}, 1 \mathrm{H})$, $5.08(\mathrm{~d}, J=11.2 \mathrm{~Hz}, 1 \mathrm{H}), 4.70(\mathrm{~d}, J=11.2 \mathrm{~Hz}, 1 \mathrm{H}), 4.50(\mathrm{dd}, J=9.3,6.3 \mathrm{~Hz}$, $1 \mathrm{H}), 4.44(\mathrm{~d}, J=11.2 \mathrm{~Hz}, 1 \mathrm{H}), 4.40(\mathrm{~d}, J=11.2 \mathrm{~Hz}, 1 \mathrm{H}), 4.32(\mathrm{~s}, 2 \mathrm{H})$, 4.12 (ddd, $J=9.8,6.8,2.9 \mathrm{~Hz}, 1 \mathrm{H}), 4.09$ (dd, $J=10.3,1.5 \mathrm{~Hz}, 1 \mathrm{H}$ ), 3.85 $(\mathrm{d}, J=2.0 \mathrm{~Hz}, 1 \mathrm{H}), 3.81(\mathrm{~d}, J=9.8 \mathrm{~Hz}, 1 \mathrm{H}), 3.79(\mathrm{dd}, J=9.8,2.4 \mathrm{~Hz}, 1 \mathrm{H})$, $3.44-3.33(\mathrm{~m}, 4 \mathrm{H}), 3.27(\mathrm{~s}, 3 \mathrm{H}), 3.14(\mathrm{dd}, J=12.2,3.0 \mathrm{~Hz}, 1 \mathrm{H}), 2.87$ (ddd, $J=14.2,7.8,4.9 \mathrm{~Hz}, 1 \mathrm{H}), 2.31-2.06(\mathrm{~m}, 5 \mathrm{H}), 2.04-1.94(\mathrm{~m}, 3 \mathrm{H}), 1.84-1.70$ $(\mathrm{m}, 2 \mathrm{H}), 1.64-1.54(\mathrm{~m}, 3 \mathrm{H}), 1.52(\mathrm{~s}, 3 \mathrm{H}), 1.34(\mathrm{~s}, 3 \mathrm{H}), 1.24(\mathrm{~s}, 3 \mathrm{H}), 1.03$ $(\mathrm{t}, J=7.8 \mathrm{~Hz}, 9 \mathrm{H}), 0.69(\mathrm{q}, J=7.8 \mathrm{~Hz}, 6 \mathrm{H}) ;{ }^{13} \mathrm{C} \mathrm{NMR}\left(100 \mathrm{MHz}, \mathrm{C}_{6} \mathrm{D}_{6}\right) \delta$ $213.9,160.9,140.2,140.1,139.9,131.6,128.9,128.8,128.5,128.0,127.9,127.9$, $127.8,127.7,114.1,103.1,85.4,83.3,82.9,81.4,78.0,77.9,77.4,76.0,73.9$, $73.2,73.2,71.1,69.7,63.9,55.1,44.0,40.2,35.4,28.9,27.8,26.0,21.5,21.1$, 20.2, 17.6, 7.6, 5.9; IR (neat) 2934, 2876, 1704, 1616, 1496, 1378, 1249, $1105 \mathrm{~cm}^{-1}$; ESI/MS $(\mathrm{m} / \mathrm{z})$ calcd for $\mathrm{C}_{58} \mathrm{H}_{78} \mathrm{O}_{11} \mathrm{SiK} 1017.6\left(\mathrm{M}+\mathrm{K}^{+}\right)$, found 1017.5.

Summary of COSY spectrum for $\mathbf{4 4}$

1. Proton at 4.50 p.p.m. (C-28) shows cross peaks with proton at 2.28 p.p.m. (C-29).

2. Proton at 4.12 p.p.m. (C-30) shows cross peaks with proton at 3.79 p.p.m. (C-31).

3. Proton at 4.09 p.p.m. (C-34) shows cross peaks with protons at 1.62 p.p.m. (C-35).

4. Proton at 3.81 p.p.m. (C-18) shows cross peaks with proton at 3.40 p.p.m. (C-18).

5. Proton at 2.06 p.p.m. (C-21) shows cross peaks with proton at 3.36 p.p.m. (C-22) and $3.14(\mathrm{C}-20)$.

Summary of 1D nOe spectrum for $\mathbf{4 4}$

1. Irradiation at 4.50 p.p.m. (C-28) resulted in enhancement at 3.36 p.p.m. (C-22).

2. Irradiation at 3.14 p.p.m. (C-20) resulted in enhancement at 5.36 p.p.m. (C-a), 3.40 p.p.m. (C-18'), and 3.36 p.p.m. (C-22).

$(2 R, 4 a R, 5 a S, 8 S, 10 S, 11 a R, 12 a S)-10-(((2 R, 3 R, 4 R, 5 S, 6 S)-4,5-b i s$ (benzyloxy)-6(3-(benzyloxy)propyl)-3-hydroxy-5-methyltetrahydro-2H-pyran-2-yl)methyl)-2(4-methoxyphenyl)-4a,5a,8-trimethyloctahydro-4H-[1,3]dioxino $\left[4^{\prime}, 5^{\prime}: 5,6\right]$ pyrano [3,2-b]oxocin-9(10H)-one (45). To a solution of $44(5.0 \mathrm{mg}, 5.1 \mu \mathrm{mol})$ in THF $(4 \mathrm{ml})$ at $-78^{\circ} \mathrm{C}$ was added LiHMDS $(0.102 \mathrm{ml}$ of $1.0 \mathrm{M}$ solution in THF, $0.10 \mathrm{mmol})$. After $10 \mathrm{~min}$, MeI ( $16 \mu \mathrm{l}, 0.254 \mathrm{mmol})$ was added and the reaction mixture was allowed to slowly warm up to $-22{ }^{\circ} \mathrm{C}$ before the reaction was quenched with $\mathrm{NH}_{4} \mathrm{Cl}$ (aq., $5 \mathrm{ml}$ ). The aqueous phase was extracted with $\mathrm{CH}_{2} \mathrm{Cl}_{2}(3 \times 5 \mathrm{ml})$ and the combined organic extracts were dried $\left(\mathrm{Na}_{2} \mathrm{SO}_{4}\right)$ and concentrated. Flash chromatography (5:1 hexanes:ethyl acetate) provided 45 $(4.7 \mathrm{mg}, 93 \%)$ as a colorless oil. $R_{\mathrm{f}} 0.55$ (3:1 hexanes:ethyl acetate). [a] ${ }^{20}{ }_{\mathrm{D}}=$ $-22.3^{\circ}\left(c=0.14\right.$, THF); ${ }^{1} \mathrm{H}$ NMR $\left(400 \mathrm{MHz}, \mathrm{CD}_{2} \mathrm{Cl}_{2}\right) \delta 7.36(\mathrm{~d}, J=8.8 \mathrm{~Hz}$, $2 \mathrm{H}), 7.32-7.19(\mathrm{~m}, 15 \mathrm{H}), 6.87(\mathrm{~d}, J=8.3 \mathrm{~Hz}, 2 \mathrm{H}), 5.44(\mathrm{~s}, 1 \mathrm{H}), 4.96(\mathrm{~d}$,
$J=10.7 \mathrm{~Hz}, 1 \mathrm{H}), 4.58(\mathrm{~d}, J=11.2 \mathrm{~Hz}, 1 \mathrm{H}), 4.54(\mathrm{~d}, J=11.2 \mathrm{~Hz}, 1 \mathrm{H}), 4.48(\mathrm{~d}$, $J=11.2 \mathrm{~Hz}, 1 \mathrm{H}), 4.46(\mathrm{~s}, 2 \mathrm{H}), 4.32(\mathrm{dd}, J=9.8,5.9 \mathrm{~Hz}, 1 \mathrm{H}), 3.85(\mathrm{~d}, J=1.5 \mathrm{~Hz}$, $1 \mathrm{H}), 3.79(\mathrm{~s}, 3 \mathrm{H}), 3.74(\mathrm{~d}, J=9.8 \mathrm{~Hz}, 1 \mathrm{H}), 3.68-3.52(\mathrm{~m}, 3 \mathrm{H}), 3.52-3.40(\mathrm{~m}$, $5 \mathrm{H}), 2.99$ (ddd $J=7.8,7.8,7.8 \mathrm{~Hz}, 1 \mathrm{H}), 2.60-2.49(\mathrm{~m}, 1 \mathrm{H}), 2.35-2.19(\mathrm{~m}, 2 \mathrm{H})$, 2.13 (ddd, $J=11.7,3.9,3.9 \mathrm{~Hz}, 1 \mathrm{H}), 1.95-1.50(\mathrm{~m}, 8 \mathrm{H}), 1.50(\mathrm{~s}, 3 \mathrm{H}), 1.36(\mathrm{~s}$, $3 \mathrm{H}), 1.28(\mathrm{~s}, 3 \mathrm{H}), 1.20(\mathrm{~d}, J=7.3 \mathrm{~Hz}, 3 \mathrm{H}), 1.03(\mathrm{t}, J=7.8 \mathrm{~Hz}, 9 \mathrm{H}), 0.71(\mathrm{q}$, $J=7.8 \mathrm{~Hz}, 6 \mathrm{H}) ;{ }^{13} \mathrm{C}$ NMR $\left(100 \mathrm{MHz}, \mathrm{C}_{6} \mathrm{D}_{6}\right) \delta 214.2,160.4,139.6,139.5,131.1$, $128.4,128.3,128.0,127.5,127.4,127.3,127.2,113.6,102.6,85.4,83.5,82.4$, $80.5,77.4,77.4,77.0,76.9,75.5,74.8,73.1,72.6,70.5,69.2,63.4,57.6,54.6$, $49.9,44.7,35.8,30.0,29.2,28.4,27.3,25.5,21.6,21.3,19.4,17.2,7.1,5.3$; IR (neat) 2956, 2876, 1718, 1616, 1496, 1377, 1249, $1104 \mathrm{~cm}^{-1}$; ESI/MS $(\mathrm{m} / \mathrm{z}) \mathrm{calcd}$ for $\mathrm{C}_{59} \mathrm{H}_{80} \mathrm{O}_{11} \mathrm{SiNa} 1015.6\left(\mathrm{M}+\mathrm{Na}^{+}\right)$, found 1015.5.

$(2 S, 3 R, 4 a S, 7 S, 7 a R, 8 a R, 9 R, 10 S, 11 S, 12 a R, 13 a S, 14 a R)-9,10-b i s($ benzyloxy)-11-(3(benzyloxy)propyl)-3-(hydroxymethyl)-3,4a,7,10-tetramethylhexadecahydropyrano [3,2-b]pyrano[2',3':5,6]pyrano[2,3-g]oxocin-2-ol (4). To a solution of 45 $(4.7 \mathrm{mg}, 4.7 \mu \mathrm{mol})$ in $\mathrm{CH}_{2} \mathrm{Cl}_{2}(3 \mathrm{ml})$ at $-78^{\circ} \mathrm{C}$ were added $\mathrm{Et}_{3} \mathrm{SiH}(76 \mu \mathrm{l}$, $0.47 \mathrm{mmol})$ and TMSOTf $(8.6 \mu \mathrm{l}, 47.0 \mu \mathrm{mol})$. After stirring for $1 \mathrm{~h}$, the reaction mixture was slowly warmed to $-50^{\circ} \mathrm{C}$, at which temperature the reaction was quenched with $\mathrm{NaHCO}_{3}$ (aq., $5 \mathrm{ml}$ ). The aqueous phase was extracted with $\mathrm{CH}_{2} \mathrm{Cl}_{2}(3 \times 5 \mathrm{ml})$ and the combined organic extracts were dried $\left(\mathrm{Na}_{2} \mathrm{SO}_{4}\right)$ and concentrated. Flash chromatography (2:1 hexanes:ethyl acetate) provided 4 (2.8 mg, $80 \%)$ as a colorless oil. $R_{\mathrm{f}} 0.45$ (1:1 hexanes:ethyl acetate); $[\alpha]^{20}{ }_{\mathrm{D}}=-1.1^{\circ}(c=0.05, \mathrm{THF}) ;{ }^{1} \mathrm{H}$ NMR $\left(500 \mathrm{MHz}, \mathrm{CD}_{2} \mathrm{Cl}_{2}\right) \delta 7.40-7.20$ $(\mathrm{m}, 15 \mathrm{H}), 4.96(\mathrm{~d}, J=11.2 \mathrm{~Hz}, 1 \mathrm{H}), 4.56(\mathrm{~d}, J=11.2 \mathrm{~Hz}, 1 \mathrm{H}), 4.45$ (d, $J=11.2 \mathrm{~Hz}, 1 \mathrm{H}), \quad 4.43 \quad(\mathrm{~s}, \quad 2 \mathrm{H}), \quad 4.40 \quad(\mathrm{~d}, J=11.2 \mathrm{~Hz}, \quad 1 \mathrm{H}), \quad 4.04$ $(\mathrm{d}, J=2.0 \mathrm{~Hz}, 1 \mathrm{H}), 3.82($ br d, $J=11.7 \mathrm{~Hz}, 1 \mathrm{H}), 3.71-3.64(\mathrm{~m}, 2 \mathrm{H})$, $3.49-3.46(\mathrm{~m}, 2 \mathrm{H}), 3.41-3.35(\mathrm{~m}, 2 \mathrm{H}), 3.29(\mathrm{~d}, J=10.7 \mathrm{~Hz}, 1 \mathrm{H}), 3.22$ (ddd, $J=11.7,8.8,4.4 \mathrm{~Hz}, 1 \mathrm{H}), 3.12(\mathrm{dd}, J=9.8,2.0 \mathrm{~Hz}, 1 \mathrm{H}), 2.76(\mathrm{dd}, J=9.3$, $9.3 \mathrm{~Hz}, 1 \mathrm{H}), 2.30$ (ddd, $J=11.2,4.4,4.4 \mathrm{~Hz}, 1 \mathrm{H}), 2.06-2.00(\mathrm{~m}, 2 \mathrm{H}), 1.96$ (ddd, $J=10.7,4.4,4.4 \mathrm{~Hz}, 1 \mathrm{H}), 1.95-1.35(\mathrm{~m}, 11 \mathrm{H}), 1.28(\mathrm{~s}, 3 \mathrm{H}), 1.22(\mathrm{~s}, 3 \mathrm{H})$, $1.14(\mathrm{~s}, 3 \mathrm{H}), 1.08$ (d, $J=6.8 \mathrm{~Hz}, 3 \mathrm{H}) ;{ }^{13} \mathrm{C}$ NMR $\left(100 \mathrm{MHz}, \mathrm{C}_{6} \mathrm{D}_{6}\right) \delta 140.1$, 128.9, 128.8, 128.0, 127.9, 127.8, 127.7, 88.1, 86.2, 83.8, 80.8, 78.8, 78.7, 77.7, 77.2, 76.6, 75.0, 73.2, 71.1, 71.0, 69.1, 68.7, 63.8, 46.3, 40.7, 40.0, 33.9, 32.1, $30.5,27.9,26.0,22.7,20.3,18.8,17.9$; IR (neat) $3500,3028,2925,1599,1494$, $1451,1264,1077 \mathrm{~cm}^{-1}$; ESI/MS $(\mathrm{m} / \mathrm{z})$ calcd for $\mathrm{C}_{45} \mathrm{H}_{60} \mathrm{O}_{9} \mathrm{~K} 783.4\left(\mathrm{M}+\mathrm{K}^{+}\right)$, found 783.3.

Summary of COSY spectrum for 4

1. Proton at 4.04 p.p.m. (C-32) shows cross peaks with proton at 3.12 p.p.m. (C-31).

2. Proton at 3.12 p.p.m. (C-31) shows cross peaks with proton at 3.66 p.p.m. (C-30).

3. Proton at 1.08 p.p.m. (C-26 methyl) shows cross peaks with protons at 1.70 p.p.m. (C-26).

4. Proton at 1.70 p.p.m. (C-26) shows cross peaks with proton at 2.76 p.p.m. (C-27).

5. Proton at 2.76 p.p.m. (C-27) shows cross peaks with proton at 3.22 p.p.m. (C-28).

6. Proton at 3.22 p.p.m. (C-28) shows cross peaks with protons at 2.30 p.p.m. (C-29) and 1.50 p.p.m. (C-29).

7. Protons at 2.30 p.p.m. (C-29) and 1.50 p.p.m. (C-29) both show cross peaks with proton at 3.66 p.p.m. (C-30).

Summary of 1D nOe spectrum for 4

1. Irradiation at 3.22 p.p.m. (C-28) resulted in enhancement at 3.66 p.p.m. (C-30), 3.40 p.p.m. (C-22), 2.30 p.p.m. (C-29) and 1.70 p.p.m. (C-26).

2. Irradiation at 2.76 p.p.m. (C-27) resulted in enhancement at 3.12 p.p.m. (C-31), 1.50 p.p.m. (C-29') and 1.08 p.p.m. (C-26 methyl).

\section{RESULTS AND DISCUSSION}

The synthesis of the $\mathrm{AB}$ bicyclic coupling precursor is depicted in Scheme 1. From known tricyclic substrate $5,{ }^{32}$ TBS ether formation 


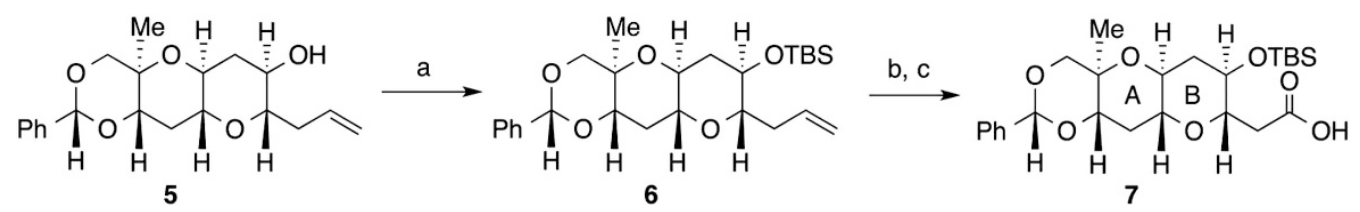

Scheme 1 Reagents and conditions: (a) TBSOTf, 2,6-lutidine, $\mathrm{CH}_{2} \mathrm{Cl}_{2}, 90 \%$; (b) $\mathrm{O}_{3}, \mathrm{CH}_{2} \mathrm{Cl}_{2},-78{ }^{\circ} \mathrm{C}$; $\mathrm{Ph}_{3} \mathrm{P},-78{ }^{\circ} \mathrm{C}$ to $\mathrm{RT}, 92 \%$; (c) $\mathrm{NaClO}_{2}, \mathrm{NaH}_{2} \mathrm{PO}_{4}$, 2-methyl-2-butene, THF-tBuOH- $\mathrm{H}_{2} \mathrm{O}, 67 \%$.

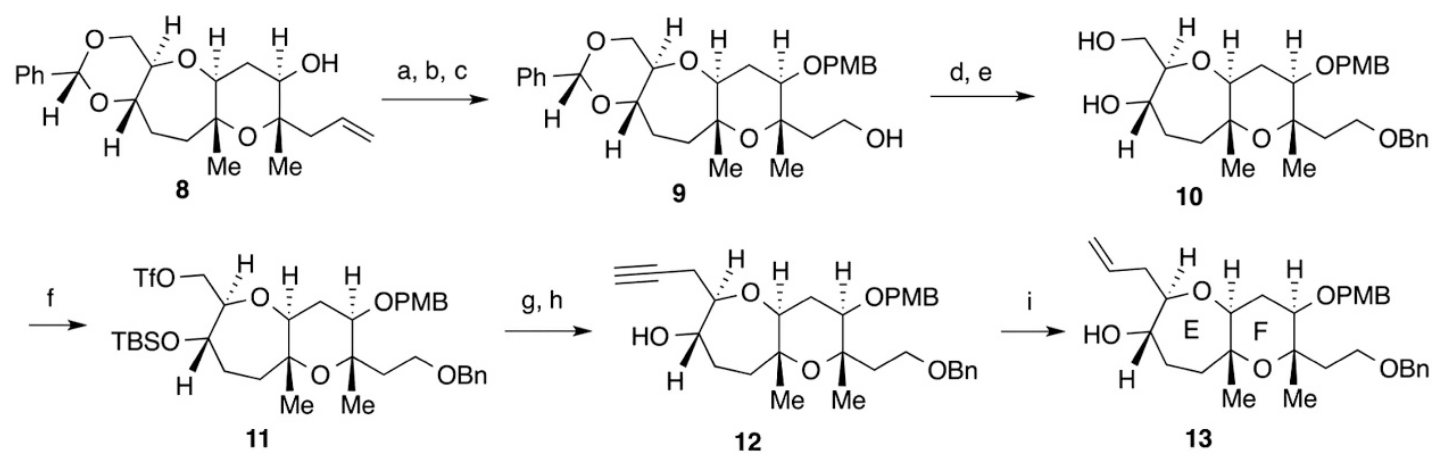

Scheme 2 Reagents and conditions: (a) $\mathrm{KH}, \mathrm{PMBBr}$, DMPU, TBAI, THF; (b) $\mathrm{O}_{3}, \mathrm{CH}_{2} \mathrm{Cl}_{2},-78{ }^{\circ} \mathrm{C}$; $\mathrm{Ph}_{3} \mathrm{P},-78{ }^{\circ} \mathrm{C}$ to $\mathrm{RT}$; (c) $\mathrm{NaBH}_{4}, \mathrm{MeOH}^{\circ} 0{ }^{\circ} \mathrm{C}, 74 \%$ (three steps); (d) tBuOK, $\mathrm{BnBr}, \mathrm{THF}, 0{ }^{\circ} \mathrm{C}$ to RT; (e) $\mathrm{CSA}, \mathrm{MeOH}, 0{ }^{\circ} \mathrm{C}$ to RT, $89 \%$ (two steps); (f) $\mathrm{Tf}_{2} \mathrm{O}, 2,6$-lutidine, $\mathrm{CH}_{2} \mathrm{Cl}_{2},-78{ }^{\circ} \mathrm{C}$; TBSOTf, $-78{ }^{\circ} \mathrm{C}$ to $\mathrm{RT}$; (g) trimethylsilylacetylene, $n \mathrm{BuLi}, \mathrm{THF}, 0^{\circ} \mathrm{C}$; $11, \mathrm{HMPA},-78^{\circ} \mathrm{C}$; (h) TBAF, THF, $75 \%$ (three steps); (i) $\mathrm{H}_{2}$, Lindlar Pd, quinoline, EtOAc, $92 \%$.

and ozonolysis gave 7 after conversion of the aldehyde into the corresponding carboxylic acid. ${ }^{33}$

Outlined in Scheme 2 is our synthesis of the EF bicyclic coupling precursor. From tricycle $8{ }^{32} \mathrm{PMB}$ ether formation, ozonolysis and reduction of the resulting aldehyde gave primary alcohol 9. Benzyl ether generation and acetal hydrolysis gave diol 10. A single-flask conversion of the $1^{\circ}$ alcohol in $\mathbf{1 0}$ into the corresponding triflate and the $2^{\circ}$ alcohol into the corresponding TBS ether gave 11 . Displacement of the triflate in $\mathbf{1 1}$ by lithium trimethylsilylacetylide and removal of the TBS group afforded 12. ${ }^{34}$ Partial hydrogenation provided EF coupling precursor $13 .{ }^{35}$

With 7 and 13 in hand, esterification using Yamaguchi's conditions gave 14 (Scheme 3). ${ }^{36}$ When subjected to our modified TakaiUtimoto reaction conditions, 14 underwent a smooth olefinic ester cyclization to afford D ring cyclic enol ether $\mathbf{1 5}$ as the only product in $50 \%$ yield. ${ }^{37}$ On the basis of our inability to isolate by-products, we believe that the modest yield was due to the instability of $\mathbf{1 5}$ and not the inefficiency of the reaction. Treatment of $\mathbf{1 5}$ with DMDO followed by reduction of the resulting epoxide with $i \mathrm{Bu}_{2} \mathrm{AlH}$ generated secondary alcohol 16 in $60 \%$ yield as a $3: 1$ mixture of diastereomers. $^{38}$ The stereochemistry at $\mathrm{C} 12$ and $\mathrm{C} 13$ in $\mathbf{1 6}$ was established using ${ }^{1} \mathrm{H}$ NMR and the ${ }^{3} J$ value between $\mathrm{C} 12$ and $\mathrm{C} 13$ following the conversion of $\mathbf{1 6}$ into the corresponding acetate $\mathbf{1 7}$ (TYX and ATX numbering). Oxidation of the mixture of diastereomers to ketone 18 followed by removal of the TBS group afforded hemiketal 19. Treatment of 19 with $\mathrm{Zn}(\mathrm{OTf})_{2}$ and EtSH gave the corresponding $O, S$-mixed ketal as a single diastereomer with concomitant removal of the benzylidene and the PMB groups. The $\mathrm{C} 2$ and $\mathrm{C} 4$ hydroxyl groups of triol 20 were selectively converted into cyclic silylene $\mathbf{2 1}$. When $\mathbf{2 1}$ was subjected to $\mathrm{Ph}_{3} \mathrm{SnH}$ and AIBN in refluxing toluene, the O,S- mixed ketal was reduced to give 3 containing the A-F ring system of both YTX and ATX. ${ }^{39}$

Having completed the synthesis of the A-F ring system, we turned our attention to the F-I subunit. Depicted in Scheme 4 is the synthesis of the F-ring coupling precursor 30. From known alcohol 22, ${ }^{40}$ acetylation followed by olefinic ester cyclization gave cyclic enol ether 24. The C19 angular methyl group helped to direct a stereoselective epoxidation of 24 with $m$ CPBA. In situ epoxide opening with $\mathrm{MeOH}$ and allylation of the resulting alcohol generated 25 . The treatment of 25 with PPTS, pyridine, and heat initiated a Claisen rearrangement giving ketone $\mathbf{2 6}$ as a single diastereomer. ${ }^{28,41}$ The stereochemistry of the newly installed angular methyl group was confirmed through the indicated nOe correlations. Reduction of the ketone and PMB ether formation afforded 27. Although the extension of the propenyl side chain in 27 into the pentenyl side chain present in 29 was relatively inefficient, it could be carried out on a reasonable scale. Removal of the benzylidene and PMB groups followed by selective C-18, C-20 PMB acetal formation provide the F-ring coupling precursor 30.

The I-ring subunit 39 was synthesized according to the route outlined in Scheme 5. From the previously reported alcohol 31, ${ }^{42}$ tertiary alcohol formation, removal of PMB group and oxidation gave 32. Formation of the TMS ether and reduction gave alcohol 33. Hydroboration and oxidative work-up provided the $1^{\circ}$ alcohol while simultaneously removing the TMS group to give 34 . Tris-benzyl ether formation and removal of the silylene group generated diol 35 . Primary triflate formation and secondary TES ether formation gave 36. The coupling of $\mathbf{3 6}$ with lithium acetylide gave $\mathbf{3 7}$ after removal of the silyl protecting groups. TES ether formation and partial hydrogenation of the alkyne followed by ozonolysis and oxidation afforded the I-ring coupling precursor 39. 


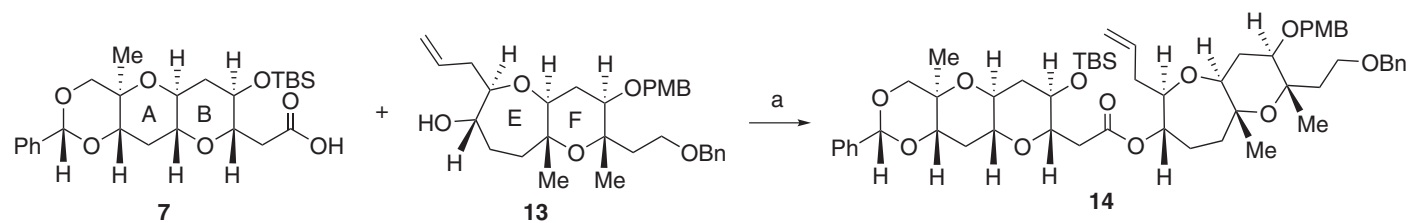

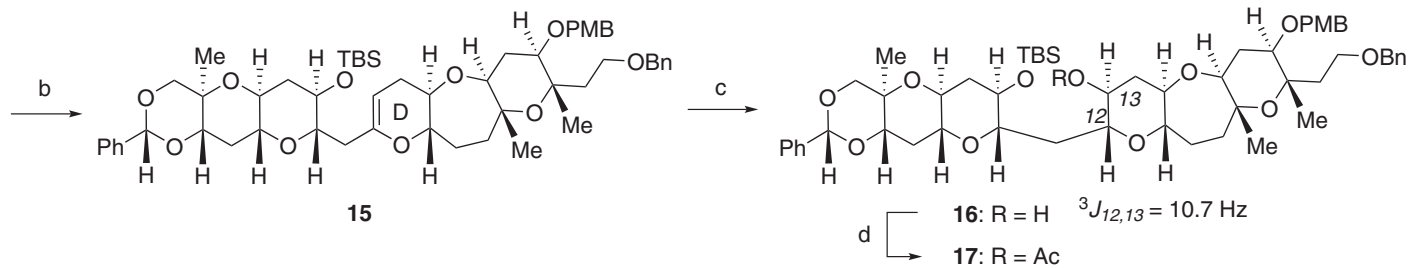

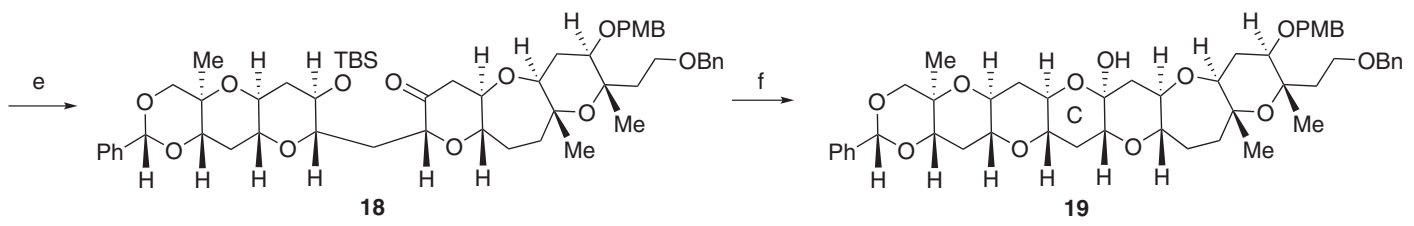

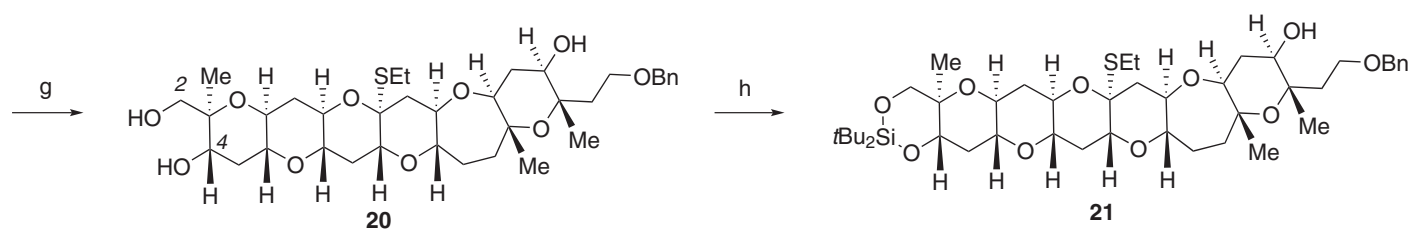
$\longrightarrow$ C

3

Scheme 3 Reagents and conditions: (a) 2,4,6- $\mathrm{Cl}_{3} \mathrm{C}_{6} \mathrm{H}_{2} \mathrm{COCl}, \mathrm{Et}_{3} \mathrm{~N}, \mathrm{THF}, 40{ }^{\circ} \mathrm{C} ; 13$, DMAP, toluene, $40{ }^{\circ} \mathrm{C}, 97 \%$; (b) $\mathrm{TiCl} 4, \mathrm{THF}, \mathrm{TMEDA}, \mathrm{Zn}, \mathrm{PbCl}{ }_{2}$, $\mathrm{CH}_{3} \mathrm{CHBr}_{2}, \mathrm{CH}_{2} \mathrm{Cl}_{2}, 65^{\circ} \mathrm{C}, 50 \%$; (c) $\mathrm{DMDO}, \mathrm{CH}_{2} \mathrm{Cl}_{2},-78^{\circ} \mathrm{C}$ to $0{ }^{\circ} \mathrm{C} ; \mathrm{Bu}_{2} \mathrm{AlH}, \mathrm{CH}_{2} \mathrm{Cl}_{2},-78{ }^{\circ} \mathrm{C}, 60 \%$ ( $+21 \%$ diastereomer); (d) $\mathrm{Ac}_{2} \mathrm{O}, \mathrm{Et} 3 \mathrm{~N}, \mathrm{DMAP}, \mathrm{CH}_{2} \mathrm{Cl}_{2}$, 81\%; (e) NMO, TPAP, $4 \AA \mathrm{MS}, \mathrm{CH}_{2} \mathrm{Cl}_{2}, 100 \%$; (f) $\mathrm{HF} \bullet P y, \mathrm{THF}, 0{ }^{\circ} \mathrm{C}$ to RT, 99\%; (g) $\mathrm{Zn}(\mathrm{OTf})_{2}, \mathrm{EtSH}, \mathrm{CH}_{2} \mathrm{Cl}_{2}, 40{ }^{\circ} \mathrm{C}$; (h) tBu $\mathrm{Si}(\mathrm{OTf})_{2}, \mathrm{Py}, \mathrm{DMF},-20{ }^{\circ} \mathrm{C}$; (i) $\mathrm{Ph}_{3} \mathrm{SnH}$, AlBN, toluene, $110{ }^{\circ} \mathrm{C}, 51 \%$ (three steps).

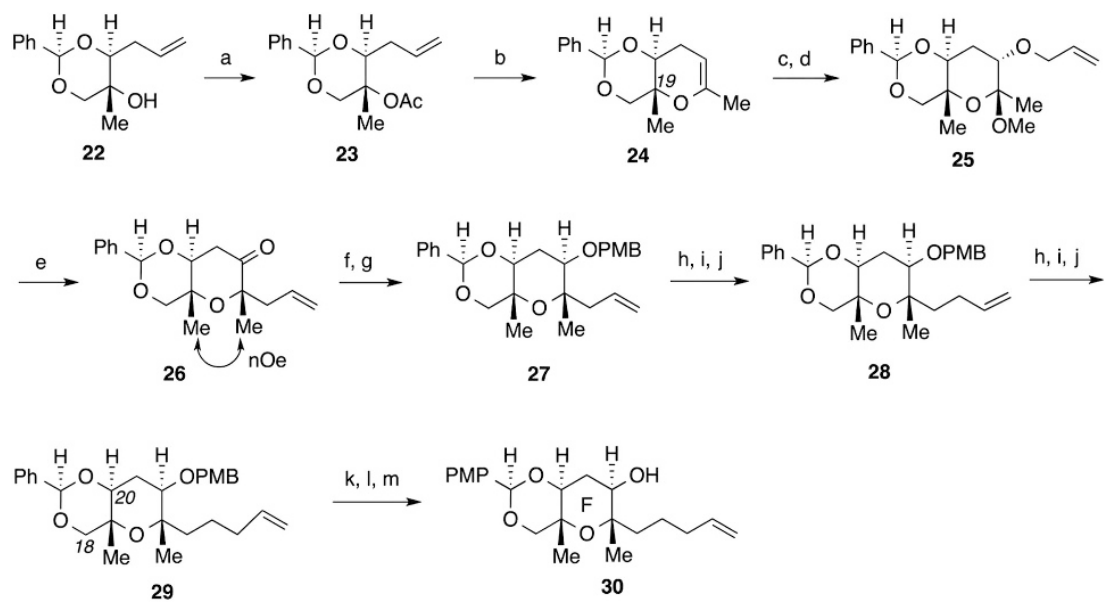

Scheme 4 Reagents and conditions: (a) $\mathrm{Ac}_{2} \mathrm{O}, \mathrm{Et}_{3} \mathrm{~N}$, DMAP, $\mathrm{CH}_{2} \mathrm{Cl}_{2}, 79 \%$; (b) $\mathrm{TiCl}_{4}$, THF, TMEDA, $\mathrm{Zn}, \mathrm{PbCl}_{2}, \mathrm{CH}_{3} \mathrm{CHBr}_{2}, \mathrm{CH}_{2} \mathrm{Cl}_{2}, 65{ }^{\circ} \mathrm{C}, 92 \%$; (c) $m \mathrm{mPBA}^{\circ}$, $\mathrm{MeOH},-78{ }^{\circ} \mathrm{C}$ to $0{ }^{\circ} \mathrm{C}, 80 \%$; (d) $\mathrm{NaH}$, allylbromide, TBAI, DMF, $0{ }^{\circ} \mathrm{C}$ to $65^{\circ} \mathrm{C}$; (e) PPTS, Py, toluene, $110{ }^{\circ} \mathrm{C}, 72 \%$ (two steps); (f) $\mathrm{NaBH}$, $\mathrm{MeOH},-78{ }^{\circ} \mathrm{C}$ to RT, $100 \%$; (g) $\mathrm{NaH}, \mathrm{PMBBr}$, TBAI, HMPA, THF, $0{ }^{\circ} \mathrm{C}$ to RT, $83 \%$; (h) $\mathrm{BH}_{3} \bullet \mathrm{DMS}, \mathrm{THF}, 0{ }^{\circ} \mathrm{C}$; $\mathrm{NaOH}, \mathrm{H}_{2} \mathrm{O}_{2}, 0{ }^{\circ} \mathrm{C}$ to RT; (i) NMO, TPAP, $4 \AA$ MS, $\mathrm{CH}{ }_{2} \mathrm{Cl}{ }_{2}$; (j) $\mathrm{Ph}_{3} \mathrm{PCH}_{3} \mathrm{Br}$, tBuOK, THF, 61\% (three steps) for 28, 55\% (three steps) for 29; (k) CSA, MeOH, $0{ }^{\circ} \mathrm{C}$ to $\mathrm{RT}, 86 \%$; (I) $\mathrm{DDQ}, \mathrm{CH}_{2} \mathrm{Cl}_{2}-\mathrm{H}_{2} \mathrm{O}, 96 \%$; (m) $p-\mathrm{MeOC}_{6} \mathrm{H}_{4} \mathrm{CH}(\mathrm{OMe})_{2}, \mathrm{CSA}, \mathrm{CH}_{2} \mathrm{Cl}_{2}, 88 \%$. 


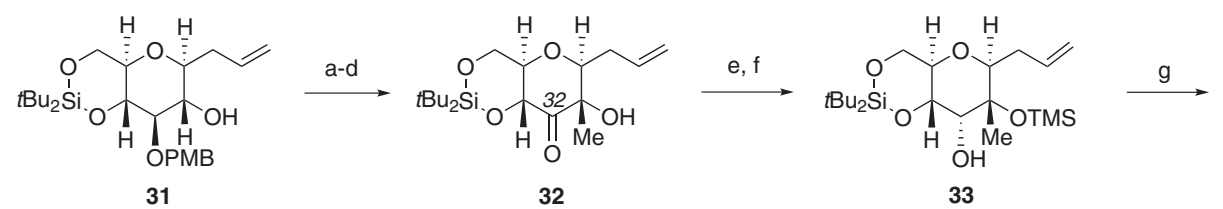

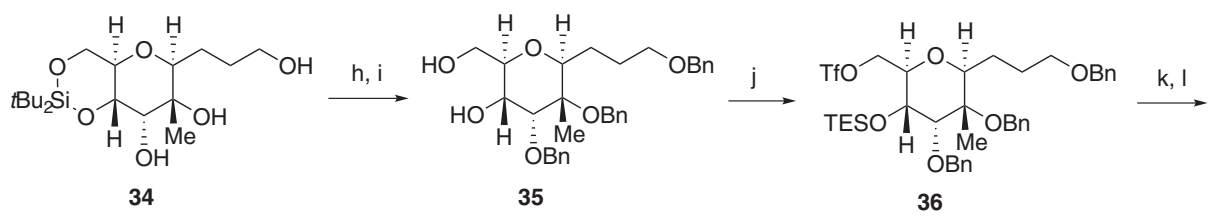

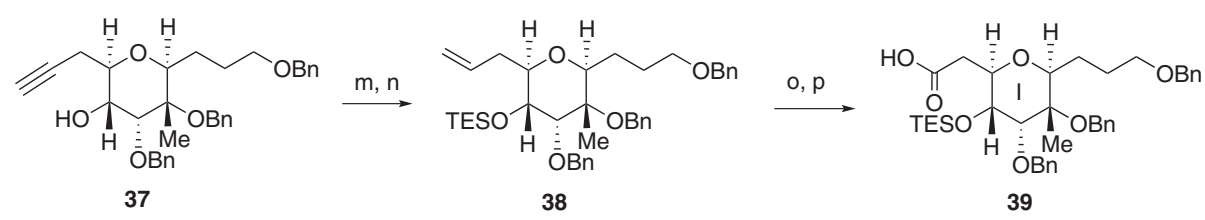

Scheme 5 Reagents and conditions: (a) $(\mathrm{COCl})_{2}$, DMSO, $\mathrm{Et}_{3} \mathrm{~N}, \mathrm{CH}_{2} \mathrm{Cl}_{2},-78{ }^{\circ} \mathrm{C}, 98 \%$; (b) $\mathrm{MeLi}$, toluene, $-90{ }^{\circ} \mathrm{C}, 84 \%$; (c) $\mathrm{DDQ}, \mathrm{CH}_{2} \mathrm{Cl}_{2}-\mathrm{H}_{2} \mathrm{O}, 95 \%$; (d) $\mathrm{SO}_{3} \bullet \mathrm{Py}, \mathrm{DMSO}, \mathrm{Et}_{3} \mathrm{~N}, \mathrm{CH}_{2} \mathrm{Cl}_{2}, 91 \%$; (e) TMSOTf, 2,6-lutidine, $\mathrm{CH}_{2} \mathrm{Cl}_{2}$; (f) L-selectride, THF, $-78{ }^{\circ} \mathrm{C}$ to $\mathrm{RT}$; (g) $\mathrm{BH}_{3} \bullet \mathrm{DMS}, \mathrm{THF}, \mathrm{O}^{\circ} \mathrm{C}$; $\mathrm{NaOH}, \mathrm{H}_{2} \mathrm{O}_{2}, \mathrm{O}^{\circ} \mathrm{C}$ to RT, $70 \%$ (three steps); (h) $\mathrm{KH}, \mathrm{BnBr}$, THF, $0{ }^{\circ} \mathrm{C}$ to RT; (i) $\mathrm{HF} \bullet P y, \mathrm{THF}, 0{ }^{\circ} \mathrm{C}$ to RT, $61 \%$ (two steps); (j) $\mathrm{Tf}_{2} \mathrm{O}, 2,6-\mathrm{Iutidine} \mathrm{CH}_{2} \mathrm{Cl} 2,-78{ }^{\circ} \mathrm{C}$; $\mathrm{TESOTf}$, $-78{ }^{\circ} \mathrm{C}$ to RT; (k) $n \mathrm{BuLi}$, trimethylsilylacetylene, THF, $0^{\circ} \mathrm{C}$; $\mathbf{3 6}$, HMPA, $-78^{\circ} \mathrm{C}$ to RT; (I) TBAF, THF, $59 \%$ (three steps); (m) TESOTf, $2,6-$ lutidine, $\mathrm{CH}_{2} \mathrm{Cl}_{2}, 0^{\circ} \mathrm{C}$ to RT, $100 \%$; (n) $\mathrm{H}_{2}$, Lindlar Pd, quinoline, EtOAc, $87 \%$; (o) $\mathrm{O}_{3}, \mathrm{CH}_{2} \mathrm{Cl}_{2},-78{ }^{\circ} \mathrm{C} ; \mathrm{Ph}_{3} \mathrm{P},-78^{\circ} \mathrm{C}$ to $\mathrm{RT}, 100 \%$; (p) $\mathrm{NaClO}_{2}, \mathrm{NaH}_{2} \mathrm{PO}_{4}$, 2-methyl-2-butene, THF-tBuOH- $\mathrm{H}_{2} \mathrm{O}, 100 \%$.<smiles>C=CCCC[C@]1(C)O[C@]2(C)CO[C@@H]([R16]#N)O[C@@H]2C[C@H]1O</smiles>

30

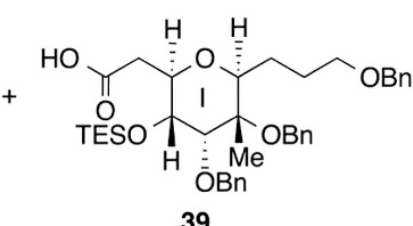

39

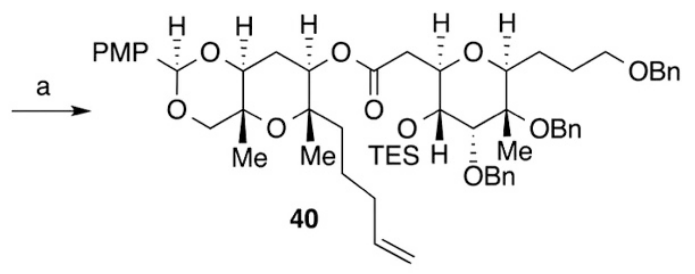

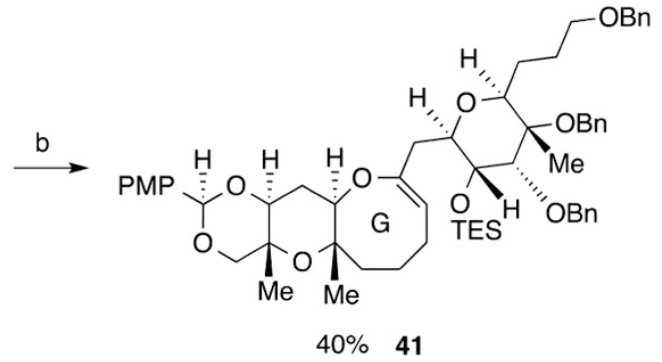

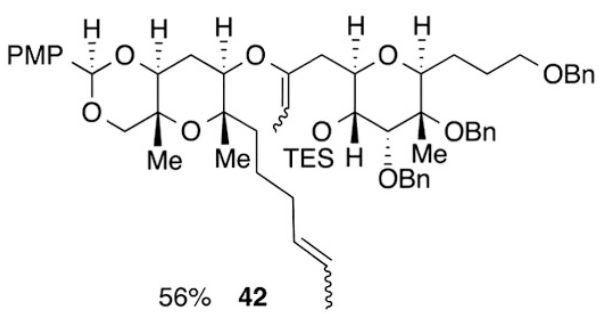

Scheme 6 Reagents and conditions: (a) 2,4,6- $\mathrm{Cl}_{3} \mathrm{C}_{6} \mathrm{H}_{2} \mathrm{COCl}, \mathrm{Et}_{3} \mathrm{~N}, \mathrm{THF}, 40{ }^{\circ} \mathrm{C} ; 30$, DMAP, toluene, $40{ }^{\circ} \mathrm{C}, 84 \%$; (b) $\mathrm{TiCl}_{4}, \mathrm{THF}, \mathrm{TMEDA}, \mathrm{Zn}, \mathrm{PbCl}{ }_{2}$, $\mathrm{CH}_{3} \mathrm{CHBr}_{2}, \mathrm{CH}_{2} \mathrm{Cl}_{2}, 65^{\circ} \mathrm{C}$.

Yamaguchi esterification using alcohol $\mathbf{3 0}$ and acid $\mathbf{3 9}$ gave ester $\mathbf{4 0}$ (Scheme 6). ${ }^{36}$ Olefinic-ester cyclization gave eight-membered G-ring cyclic enol ether $\mathbf{4 1}$ in $40 \%$ yield. We are unaware of any other reports of eight-membered cyclic enol ether formation using either olefinic-ester cyclization or olefinic enol ether cyclization conditions.

Having access to 41, we next examined the incorporation of the H-ring and the C-26 methyl group (Scheme 7). The oxidation of 41 with DMDO was followed by the in situ reduction of the resulting epoxide with $i \mathrm{Bu}_{2} \mathrm{AlH}$ giving secondary alcohol 43 as a single diastereomer. Oxidation provided ketone $\mathbf{4 4}$ whose stereochemistry was determined from the indicated nOe enhancements. The incorporation of the C-26 methyl group was accomplished through alkylation of the enolate from 44 to give 45 . Reductive cyclization of the TES-protected hydroxy ketone 45 using TMSOTf and $\mathrm{Et}_{3} \mathrm{SiH}$ resulted in the generation of the G-ring. This transformation also resulted in the removal of the PMB acetal. Interestingly, when a benzylidene acetal rather than a p-methoxy benzylidene actal was used as the protecting group on the F-ring, it could not be cleanly removed under the reductive cyclization conditions, giving instead a mixture of benzyl protected alcohols. We were able to ascertain the relative stereochemistry of $\mathbf{4}$ using the indicated nOe enhancements.

In summary, we have achieved the convergent syntheses of both the ABCDEF and FGHI ring system of YTX and ATX using our olefinic ester cyclization strategy. We have also demonstrated that eightmembered cyclic enol ethers can be generated using an olefinic ester 


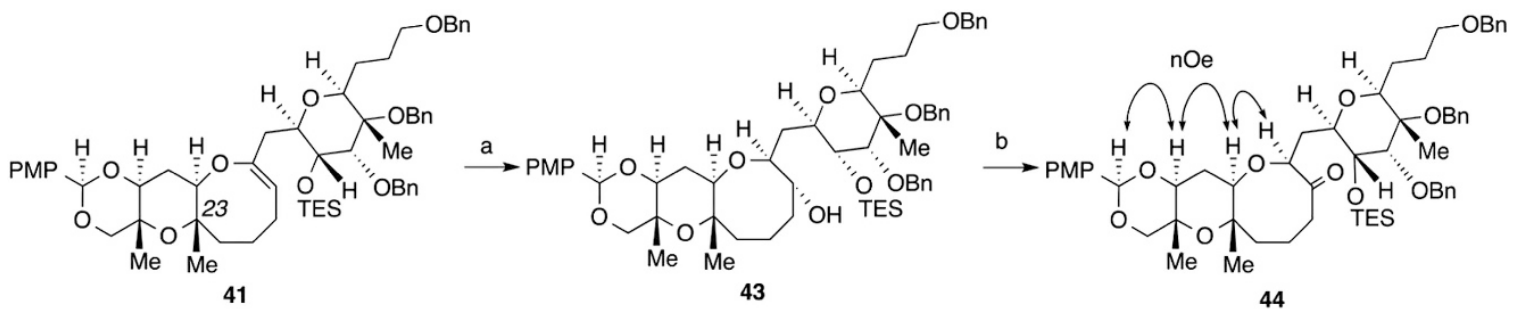
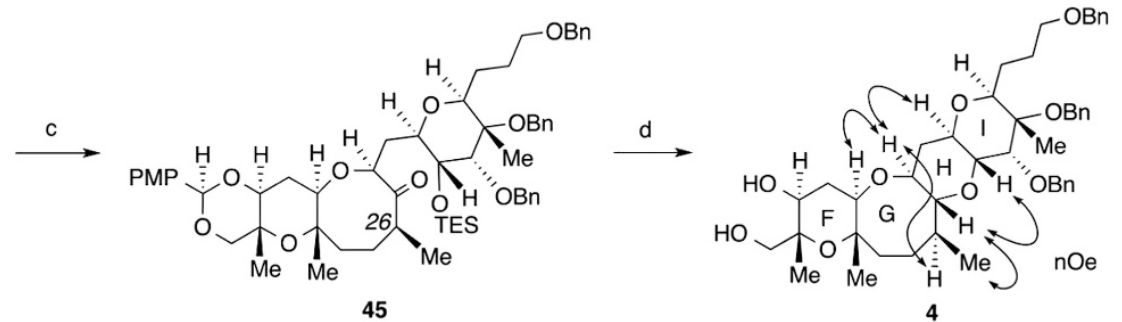

Scheme 7 Reagents and conditions: (a) DMDO, $\mathrm{CH}_{2} \mathrm{Cl}_{2},-78{ }^{\circ} \mathrm{C}$ to $0{ }^{\circ} \mathrm{C}$; $i \mathrm{Bu}_{2} \mathrm{AlH}, \mathrm{CH}_{2} \mathrm{Cl}_{2},-78^{\circ} \mathrm{C}$; (b) NMO, TPAP, $4 \AA \mathrm{AS}, \mathrm{CH}_{2} \mathrm{Cl}_{2}, 61 \%$ (two steps); (c) LiHMDS, $-78{ }^{\circ} \mathrm{C}$; Mel, $-78{ }^{\circ} \mathrm{C}$ to $-22^{\circ} \mathrm{C}, 93 \%$; (d) TMSOTf, Et ${ }_{3} \mathrm{SiH},-78{ }^{\circ} \mathrm{C}$ to $-50{ }^{\circ} \mathrm{C}, 80 \%$.

cyclization reaction. Further studies toward the total syntheses of YTX and ATX are currently in progress in our laboratory.

\section{CONFLICT OF INTEREST}

The authors declare no conflict of interest.

\section{ACKNOWLEDGEMENTS}

We thank the National Institutes of Health, General Medical Sciences (GM56677) for their support in this work. We are grateful to the staff at the University of Utah, especially Dr Peter Flynn and Dr Dennis Edwards (NMR) and Dr Jim Muller (mass spectrometry).

1 Murata, M., Kumagai, M., Masanori, K., Lee, J.-S. \& Yasumoto, T. Isolation and structure of yessotoxin, a novel polyether compound implicated in diarrhetic shellfish poisoning. Tetrahedron Lett. 28, 5869-5872 (1987).

2 Satake, M., MacKenzie, L. \& Yasumoto, T. Identification of Protoceratium reticulatum as the biogenetic origin of yessotoxin. Nat. Toxins 5, 164-167 (1997).

3 Paz, B., Riobó, P., Fernández, L. M., Fraga, S. \& Franco, J. M. Production and release of yessotoxins by the dinoflagellates Protoceratium reticulatum and Lingulodinium polyedrum in culture. Toxicon 44, 251-258 (2004).

4 Rhodes, L.V. et al. Yessotoxin production by Gonyaulax spinifera. Harmful Algae $\mathbf{5}$ 148-155 (2006).

5 Tubaro, A., Dell'Ovo, V., Sosa, S. \& Florio, C. Yessotoxins: a toxicological overview. Toxicon 56, 163-172 (2010).

6 Terao, K., Ito, E., Oarada, M., Murata, M. \& Yasumoto, T. Histopathological studies on experimental marine toxin poisoning-5. The effects in mice of yessotoxin isolated from Patinopecten yessoensis and of a desulfated derivative. Toxicon 28, 1095-1104 (1990).

7 Tubaro, A. et al. Effects of yessotoxin (YTX) on the skeletal muscle: an update. Food Addit. Contam. Part A Chem. Anal. Control Expo Risk Assess. 25, 1095-1100 (2008).

8 Tubaro, A.et al. Short-term oral toxicity of homoyessotoxins, yessotoxin and okadaic acid in mice. Toxicon 43, 439-445 (2004).

9 Tubaro, A. et al. Ultrastructural damage to heart tissue from repeated oral exposure to yessotoxin resolves in three months. Toxicon 51, 1225-1235 (2008).

10 De la Rosa, L. A., Alfonso, A., Vilariño, N., Vieytes, M. R. \& Botana, L. M. Modulation of cytosolic calcium levels of human lymphocytes by yessotoxin, a novel marine phycotoxin. Biochem. Pharmacol. 61, 827-833 (2001).

11 Ronzitti, G., Callegari, F., Malaguti, C. \& Rossini, G. P. Selective disruption of the E-cadherin-catenin system by an algal toxin. Br. J. Cancer 90, 1100-1107 (2004).

12 Ronzitti, G. \& Rossini, G. P. Yessotoxin induces the accumulation of altered E-cadherin dimers that are not part of adhesive structures in intact cells. Toxicology 244, 145-156 (2008).

13 Korsnes, M. S. \& Espenes, A. Yessotoxin as an apoptotic inducer. Toxicon 57, 947-958 (2011).
14 Malagoli, D., Marchesini, E. \& Ottaciani, E. Lysosomes as the target of yessotoxin in invertebrate and vertebrate cell lines. Toxicol. Lett. 167, 75-83 (2006).

15 Ciminiello, P.et al. Isolation of adriatoxin, a new analog of yessotoxin from mussels of the Adriatic Sea. Tetrahedron Lett. 39, 8897-8900 (1998).

16 Suzuki, K. \& Nakata, T. Convergent Synthesis of the ABCDEF-Ring System of Yessotoxin and Adriatoxin. Org. Lett. 4, 3943-3946 (2002).

17 Mori, Y., Nogami, K., Hayashi, H. \& Noyori, R. Sulfonyl-Stabilized Oxiranylithium-Based Approach to Polycyclic Ethers. Convergent Synthesis of the ABCDEF-Ring System of Yessotoxin and Adriatoxin. J. Org. Chem. 68, 9050-9060 (2003).

18 Hiramatsu, N. \& Mori, Y. Synthesis of the 37-epi-HIJ ring system of adriatoxin. Heterocycles 69, 437-446 (2006)

19 Kadota, I., Ueno, H. \& Yamamoto, Y. Convergent synthesis of the A-F ring segment of yessotoxin and adriatoxin. Tetrahedron Lett. 44, 8935-8938 (2003).

20 Kadota, I., Ueno, H., Sato, Y. \& Yamamoto, Y. Convergent synthesis of the FGHI ring segment of yessotoxin. Tetrahedron Lett. 47, 89-92 (2006).

21 Kadota, I., Abe, T., Sato, Y., Kabuto, C. \& Yamamoto, Y. Stereocontrolled synthesis of the IJK ring segment of yessotoxin. Tetrahedron Lett. 47, 6545-6548 (2006).

22 Watanabe, K., Suzuki, M., Murata, M. \& Oishi, T. Convergent synthesis of the FGHI ring system of yessotoxin: stereoselective construction of the $\mathrm{G}$ ring. Tetrahedron Lett. 46 3991-3995 (2005).

23 Oishi, T., Suzuki, M., Watanabe, K. \& Murata, M. Convergent synthesis of the CDEF ring fragment of yessotoxin via $\alpha$-cyano ethers. Heterocycles 69 , 91-98 (2006).

24 Oishi, T., Suzuki, M., Watanabe, K. \& Murata, M. Synthesis of the ABC and IJ ring fragments of yessotoxin. Tetrahedron Lett. 47, 3975-3978 (2006).

25 Watanabe, K., Minato, H., Murata, M. \& Oishi, T. Synthesis of the JK ring fragments of yessotoxin and 42,43,44,45,46,47,55-heptanor-41-oxoyessotoxin. Heterocycles $\mathbf{7 2}$, 207-212 (2007).

26 Torikai, K. et al. Convergent synthesis of the A-J ring system of yessotoxin. Synlett 2368-2372 (2008).

27 Oishi, T., Imaizumi, T. \& Murata, M. Reductive etherification under microfluidic conditions: application to practical synthesis of the FGHIJ-ring system of yessotoxin. Chem. Lett. 39, 108-109 (2010).

28 Akoto, C. O. \& Rainier, J. D. Harnessing glycal-epoxide rearrangements: the generation of the $\mathrm{AB}, \mathrm{EF}$, and IJ rings of adriatoxin. Angew. Chem. Int. Ed. 47, 8055-8058 (2008).

29 Johnson, H. W. B., Majumder, U. \& Rainier, J. D. The total synthesis of gambierol. J. Am. Chem. Soc. 127, 848-849 (2005).

30 Johnson, H. W. B., Majumder, U. \& Rainier, J. D. Total synthesis of gambierol: subunit coupling and completion. Chem. Eur. J 12, 1747-1753 (2006).

31 Zhang, Y. \& Rainier, J. D. Two-directional olefinic-ester ring-closing metathesis using reduced $\mathrm{Ti}$ alkylidenes. a rapid entry into polycyclic ether skeletons. Org. Lett. 11 237-239 (2009).

32 Zhang, Y., Rohanna, J., Zhou, J., lyer, K. \& Rainier, J. D. Total synthesis of brevenal. J. Am. Chem. Soc. 133, 3208-3216 (2011).

33 Bal, B. S., Childers, W. E. \& Pinnick, H. W. Oxidation of $\alpha, \beta$-unsaturated aldehydes. Tetrahedron 37, 2091-2096 (1981).

34 Clark, J. S., Kimber, M. C., Robertson, J., McErlean, C. S. P. \& Wilson, C. Rapid two-directional synthesis of the $\mathrm{F}$-J fragment of the gambieric acids by iterative double ring-closing metathesis. Angew Chem. Int. Ed. 44, 6157-6162 (2005).

35 Lindlar, H. A new catalyst for selective hydrogenations. Helv. Chim. Acta 35, 446-448 (1952) 
36 Inanaga, J., Hirata, K., Saeki, T., Katusuki, T. \& Yamaguchi, M. A rapid esterification by mixed anhydride and its application to large-ring lactonization. Bull. Chem. Soc. Jpn 52, 1989-1993 (1979).

37 lyer, K. \& Rainier, J. D. Olefinic ester and diene ring-closing metathesis using a reduced titanium alkylidene. J. Am. Chem. Soc. 129, 12604-12605 (2007).

38 Roberts, S. W. \& Rainier, J. D. Synthesis of an A-E gambieric acid subunit with use of a C-glycoside centered strategy. Org. Lett. 9, 2227-2230 (2007).

39 Cox, J. M. \& Rainier, J. D. C-glycosides to fused polycyclic ethers. An efficient entry into the A-D ring system of gambierol. Org. Lett. 3, 2919-2922 (2001).
40 Nicolaou, K. C., Prasad, C. V. C., Hwang, C.-K., Duggan, M. E. \& Veale, C. A. Cyclizations of hydroxyl dithioketals. New synthetic technology for the construction of oxocenes and related mediumOring systems. J. Am. Chem. Soc. 111, 5321-5330 (1989).

41 Rainier, J. D., Allwein, S. P. \& Cox, J. M. C-glycosides to fused polycyclic ethers. A formal synthesis of ( \pm )-Hemibrevetoxin B. J. Org. Chem. 66, 1380-1386 (2001).

42 Clark, J. S. \& Hamelin, O. Synthesis of polycyclic ethers by two-directional ring-closing metathesis. Angew. Chem. Int. Ed. 39, 372-374 (2000).

Supplementary Information accompanies the paper on The Journal of Antibiotics website (http://www.nature.com/ja) 